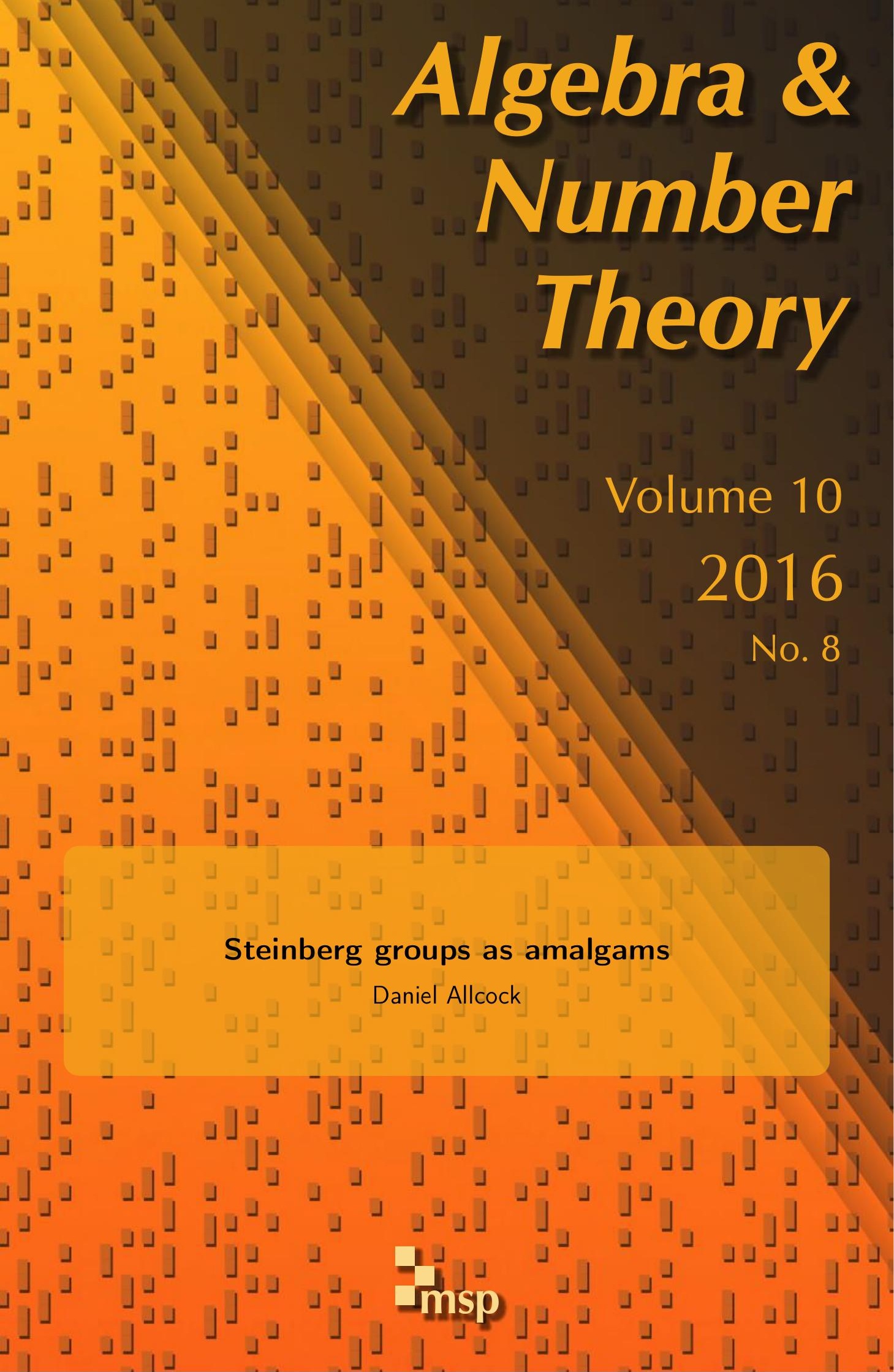




\section{Steinberg groups as amalgams}

\section{Daniel Allcock}

For any root system and any commutative ring, we give a relatively simple presentation of a group related to its Steinberg group $\mathfrak{S t}$. This includes the case of infinite root systems used in Kac-Moody theory, for which the Steinberg group was defined by Tits and Morita-Rehmann. In most cases, our group equals $\mathfrak{S t}$, giving a presentation with many advantages over the usual presentation of $\mathfrak{S t}$. This equality holds for all spherical root systems, all irreducible affine root systems of rank $>2$, and all 3-spherical root systems. When the coefficient ring satisfies a minor condition, the last condition can be relaxed to 2-sphericity.

Our presentation is defined in terms of the Dynkin diagram rather than the full root system. It is concrete, with no implicit coefficients or signs. It makes manifest the exceptional diagram automorphisms in characteristics 2 and 3 , and their generalizations to Kac-Moody groups. And it is a Curtis-Tits style presentation: it is the direct limit of the groups coming from 1- and 2-node subdiagrams of the Dynkin diagram. Over nonfields this description as a direct limit is new and surprising. Our main application is that many Steinberg and Kac-Moody groups over finitely generated rings are finitely presented.

1. Introduction

1792

2. Examples

1796

3. Diagram automorphisms 1800

4. The Kac-Moody algebra 1802

5. The extension $W^{*} \subseteq$ Aut $\mathfrak{g}$ of the Weyl group 1803

6. The Steinberg group $\mathfrak{S t} 1806$

7. The pre-Steinberg group $\mathfrak{P S t} 1812$

8. The isomorphism $\mathfrak{G}_{2} \cong\left(*_{\alpha \in \Phi} \mathfrak{U}_{\alpha}\right) \rtimes \widehat{W} \quad 1818$

9. The isomorphism $\mathfrak{G}_{3} \cong \mathfrak{P S} \mathfrak{t}^{\text {Tits }} \rtimes \widehat{W} \quad 1824$

10. The adjoint representation 1827

11. $\mathfrak{P S t} \rightarrow \mathfrak{S t}$ is often an isomorphism 1830

12. Finite presentations 1836

Acknowledgements 1842

References 1842

Supported by NSF grant DMS-1101566.

MSC2010: primary 19C99; secondary 20G44, 14L15.

Keywords: Kac-Moody group, Steinberg group, pre-Steinberg group, Curtis-Tits presentation. 


\section{Introduction}

In this paper we give a presentation for a Steinberg-like group, over any commutative ring, for any root system, finite or not. For many root systems, including all finite ones, it is the same as the Steinberg group $\mathfrak{S t}$. This is the case of interest, for then it gives a new presentation of $\mathfrak{S t}$ and associated Chevalley and Kac-Moody groups. Our presentation

(i) is defined in terms of the Dynkin diagram rather than the set of all (real) roots (Sections 2 and 7);

(ii) is concrete, with no coefficients or signs left implicit;

(iii) generalizes the Curtis-Tits presentation of Chevalley groups to rings other than fields (Corollary 1.3);

(iv) is rewritable as a finite presentation when $R$ is finitely generated as an abelian group (Theorem 1.4);

(v) is often rewritable as a finite presentation when $R$ is merely finitely generated as a ring (Theorem 1.4);

(vi) allows one to prove that many Kac-Moody groups are finitely presented (Theorem 1.5); and

(vii) makes manifest the exceptional diagram automorphisms that lead to the Suzuki and Ree groups, and allows one to construct similar automorphisms of KacMoody groups in characteristic 2 or 3 (Section 3).

More precisely, given any generalized Cartan matrix $A$, in Section 7 we give two definitions of a new group functor. We call it the pre-Steinberg group $\mathfrak{P S t}_{A}$ because it has a natural map to $\mathfrak{S t}_{A}$. This will be obvious from the first definition, which mimics Tits' definition [1987] of the Steinberg group $\mathfrak{S t}_{A}$, as refined by Morita and Rehmann [1990]. The difference is that we leave out most of the relations. If the root system is finite then both $\mathfrak{P S t}_{A}$ and $\mathfrak{S t}_{A}$ coincide with Steinberg's original group functor, so they coincide with each other too. Our perspective is that $\mathfrak{P S t}_{A}(R)$ is interesting if and only if $\mathfrak{P S t}_{A}(R) \rightarrow \mathfrak{S t}_{A}(R)$ is an isomorphism, when our second definition of $\mathfrak{P S t}_{A}$ provides a new and useful presentation of $\mathfrak{S t}_{A}$. We will discuss this second definition after listing some cases in which

$$
\mathfrak{P S t}_{A}(R) \cong \mathfrak{S t}_{A}(R) .
$$

Theorem 1.1 (coincidence of Steinberg and pre-Steinberg groups). Suppose $R$ is a commutative ring and $A$ is a generalized Cartan matrix. Then the natural map $\mathfrak{P S t}_{A}(R) \rightarrow \mathfrak{S t}_{A}(R)$ is an isomorphism in any of the following cases:

(i) if $A$ is spherical; or

(ii) if A is irreducible affine of rank $>2$; or 
(iii) if $A$ is 3-spherical; or

(iv) if $A$ is 2-spherical and (if $A$ has a multiple bond) $R$ has no quotient $\mathbb{F}_{2}$ and (if $A$ has a triple bond) $R$ has no quotient $\mathbb{F}_{3}$.

Language. We pass between Cartan matrices and Dynkin diagrams whenever convenient. The rank rk $A$ of $A$ means the number of nodes of the Dynkin diagram. $A$ is called spherical if its Weyl group is finite; this is equivalent to every component of the Dynkin diagram being one of the classical $A B C D E F G$ diagrams. $A$ is called $k$-spherical if every subdiagram with $\leq k$ nodes is spherical.

As mentioned above, case (i) in Theorem 1.1 is obvious once $\mathfrak{P S t}$ is defined. Cases (iii)-(iv) are proven in Section 11. By considering the list of affine Dynkin diagrams, one sees that these cases imply case (ii) except in rank 3 when $R$ has a forbidden $\mathbb{F}_{2}$ or $\mathbb{F}_{3}$ quotient. Proving (ii) requires removing this restriction on $R$, for which we refer to [Allcock 2016]. An early version of the present paper was used in [Allcock and Carbone 2016] to establish Theorem 1.1 for certain hyperbolic Dynkin diagrams. Those diagrams are now covered by case (iv).

Our second "definition" of $\mathfrak{P S t}_{A}(R)$ is the following theorem, giving a presentation for it. It is a restatement of Theorem 7.12, whose proof occupies Sections 7-9. The proof relies on an understanding of root stabilizers under a certain extension of the Weyl group, which appears to be a new ingredient in Lie theory. To give the flavor of the result, the full presentation appears in Table 1.1 if $A$ is simply laced without $A_{1}$ components. In this case we have $\mathfrak{P S t}_{A}(R)=\mathfrak{S t}_{A}(R)$ by the previous theorem, so it is a new presentation for $\mathfrak{S t}_{A}(R)$.

Theorem 1.2 (presentation of pre-Steinberg groups). For any commutative ring $R$ and any generalized Cartan matrix $A, \mathfrak{P S t}_{A}(R)$ has a presentation with generators $S_{i}$ and $X_{i}(t)$, where $i$ varies over the simple roots and $t$ varies over $R$, and relators (7-1)-(7-26).

Table 1.1 shows that the presentation is less intimidating than a list of 26 relations would suggest. See Section 2 for the $B_{2}$ and $G_{2}$ cases. Each relator (7-1)-(7-26) involves at most two distinct subscripts. This proves the following.

Corollary 1.3 (Curtis-Tits presentation for pre-Steinberg groups). Let $A$ be a generalized Cartan matrix and $R$ a commutative ring. Consider the groups $\mathfrak{P S t}_{B}(R)$ and the obvious maps between them, as $B$ varies over the $1 \times 1$ and $2 \times 2$ submatrices of A coming from singletons and pairs of nodes of the Dynkin diagram. The direct limit of this family of groups equals $\mathfrak{P S t}_{A}(R)$.

In any of the cases in Theorem 1.1, we may replace $\mathfrak{P S t}_{A}$ by $\mathfrak{S t}_{A}$ everywhere in Corollary 1.3, yielding a Curtis-Tits style presentation for $\mathfrak{S t}_{A}$. This is the source of our title Steinberg groups as amalgams. We learned after writing this paper that Dennis and Stein [1974, Theorem B] announced Corollary 1.3 for finite root 


\begin{tabular}{|c|c|}
\hline all $i$ & $\begin{aligned} X_{i}(t) X_{i}(u) & =X_{i}(t+u) \\
{\left[S_{i}^{2}, X_{i}(t)\right] } & =1 \\
S_{i} & =X_{i}(1) S_{i} X_{i}(1) S_{i}^{-1} X_{i}(1)\end{aligned}$ \\
\hline all $(i, j)$ with $i \neq j$ unjoined & $\begin{aligned} S_{i} S_{j} & =S_{j} S_{i} \\
{\left[S_{i}, X_{j}(t)\right] } & =1 \\
{\left[X_{i}(t), X_{j}(u)\right] } & =1\end{aligned}$ \\
\hline all $(i, j)$ with $i \neq j$ joined & $\begin{aligned} S_{i} S_{j} S_{i} & =S_{j} S_{i} S_{j} \\
S_{i}^{2} S_{j} S_{i}^{-2} & =S_{j}^{-1} \\
X_{i}(t) S_{j} S_{i} & =S_{j} S_{i} X_{j}(t) \\
S_{i}^{2} X_{j}(t) S_{i}^{-2} & =X_{j}(t)^{-1} \\
{\left[X_{i}(t), S_{i} X_{j}(u) S_{i}^{-1}\right] } & =1 \\
{\left[X_{i}(t), X_{j}(u)\right] } & =S_{i} X_{j}(t u) S_{i}^{-1}\end{aligned}$ \\
\hline
\end{tabular}

Table 1.1. Our defining relations for the Steinberg group $\mathfrak{S t}_{A}(R)$, when $A$ is any simply laced generalized Cartan matrix, without $A_{1}$ components, and $R$ is any commutative ring. The generators are $X_{i}(t)$ and $S_{i}$, where $i$ varies over the nodes of the Dynkin diagram and $t$ over $R$.

systems. They did not publish a proof, and from their announcement it appears that their approach was not via our Theorem 1.2.

In the $A_{1}, A_{2}, B_{2}$ and $G_{2}$ cases, we write out our presentation of $\mathfrak{P S t}_{A}(R)=$ $\mathfrak{S t}_{A}(R)$ explicitly in Section 2. We do this to make our results as accessible as possible, and to show in Section 3 that our presentation makes manifest the exceptional diagram automorphisms in characteristics 2 and 3. Namely, the arrowreversing diagram automorphism of the $B_{2}$ or $G_{2}$ Dynkin diagram yields a selfhomomorphism of the corresponding Steinberg group if the coefficient ring $R$ has characteristic 2 or 3 , respectively. If $R$ is a perfect field then this self-homomorphism is the famous outer automorphism that leads to the Suzuki and (small) Ree groups.

Because of the direct limit property (Corollary 1.3), one obtains the corresponding self-homomorphisms of $F_{4}$ in characteristic 2 with no more work. That is, the defining relations for $\mathfrak{S t}_{F_{4}}$ are those for $\mathfrak{S t}_{B_{2}}$, two copies of $\mathfrak{S t}_{A_{2}}$ and three copies of

$$
\mathfrak{S t}_{A_{1}^{2}}=\mathfrak{S t}_{A_{1}} \times \mathfrak{S t}_{A_{1}} .
$$

The diagram automorphism transforms the $B_{2}$ relations as in the previous paragraph and sends the other relations into each other. The same argument applies to many Kac-Moody groups. By work of Hée, this leads to Kac-Moody-like analogues of the Suzuki and Ree groups, discussed briefly in Section 3. 
An application of the theory we have described is that Steinberg groups and Kac-Moody groups are finitely presented under quite weak hypotheses on their Dynkin diagrams and coefficient rings. We state the Steinberg group result in terms of $\mathfrak{P S t}_{A}(R)$, keeping in mind that the interesting case is when $\mathfrak{P S t}_{A}(R)$ coincides with $\mathfrak{S t}_{A}(R)$. See Section 12 for the proof.

Theorem 1.4 (finite presentation of pre-Steinberg groups). Let $R$ be a commutative ring and $A$ a generalized Cartan matrix. Then $\mathfrak{P S t}_{A}(R)$ is finitely presented in any of the following cases:

(i) if $R$ is finitely generated as an abelian group; or

(ii) if $A$ is 2-spherical without $A_{1}$ components, and $R$ is finitely generated as a module over a subring generated by finitely many units; or

(iii) if $R$ is finitely generated as a ring, and any two nodes of A lie in an irreducible spherical diagram of rank $\geq 3$.

Many authors have studied the finite presentation of Steinberg groups and related groups. Our Theorem 1.4 is inspired by work of Splitthoff [1986]. See [Kiralis et al. 1996; Zhang 1991; Li 1989] for some additional results.

The Kac-Moody group version of Theorem 1.4 concerns the group functors $\mathfrak{G}_{D}$ constructed by Tits [1987] (he wrote $\widetilde{\mathfrak{G}}_{D}$ ). They were his motivation for generalizing the Steinberg groups beyond the case of spherical Dynkin diagrams. He defined the "simply connected" Kac-Moody groups as certain quotients of Steinberg groups, and arbitrary Kac-Moody groups are only slightly more general. Specifying a KacMoody group requires specifying a root datum $D$, which is slightly more refined information than $D$ 's associated generalized Cartan matrix $A$. But the choice of $D$ doesn't affect any of our results.

Our final theorem shows that a great many Kac-Moody groups over rings are finitely presented. This is surprising because one thinks of Kac-Moody groups over (say) $\mathbb{R}$ as infinite-dimensional Lie groups, so the same groups over (say) $\mathbb{Z}$ should be some sort of discrete subgroups. There is no obvious reason why a discrete subgroup of an infinite-dimensional Lie group should be finitely presented. See Section 12 for the definition of the Kac-Moody groups, and the proof of the following theorem.

Theorem 1.5 (finite presentation of Kac-Moody groups). Suppose A is a generalized Cartan matrix and $R$ is a commutative ring whose group of units $R^{*}$ is finitely generated. Let $D$ be any root datum with generalized Cartan matrix A. Then Tits' Kac-Moody group $\mathfrak{G}_{D}(R)$ is finitely presented if $\mathfrak{S t}_{A}(R)$ is.

In particular, this holds if one of (i)-(iv) from Theorem 1.1 holds and one of (i)-(iii) from Theorem 1.4 holds. 
The paper is organized as follows. Sections 2 and 3 are expository and not essential for later sections. Section 2 is really a continuation of the introduction, writing down the essential cases of our presentation of $\mathfrak{P S t}_{A}(R)$. These can be understood independently of the rest of the paper. Section 3 treats the exceptional diagram automorphisms: their existence is hardly even an exercise.

Sections 4-6 give necessary background. Section 4 gives a little background on the Kac-Moody algebra $\mathfrak{g}_{A}$. Section 5 is mostly a review of results of Tits about a certain extension $W^{*} \subseteq \operatorname{Aut}\left(\mathfrak{g}_{A}\right)$ of the Weyl group $W$. But we also use a more recent result of Brink [1996] on Coxeter groups to describe generators for root stabilizers in $W^{*}$, and how they act on the corresponding root spaces (Theorem 5.7). Section 6 reviews Tits' definition of $\mathfrak{S t}_{A}$ and its refinement by Morita and Rehmann.

Sections 7-9 are the technical heart of the paper, establishing Theorem 1.2. In Section 7 we define $\mathfrak{P S t}_{A}$ and then establish a presentation for it. We do this by defining a group functor $\mathfrak{G}_{4}$ by a presentation and proving $\mathfrak{P S t}_{A} \cong \mathfrak{G}_{4}$. As the notation suggests, this is the last in a chain of group functors $\mathfrak{G}_{1}, \ldots, \mathfrak{G}_{4}$ that give successively better approximations to $\mathfrak{P S t}_{A}$. Lemma 7.4 and Theorems 7.5, 7.11 and 7.12 give "intrinsic" descriptions of $\mathfrak{G}_{1}, \mathfrak{G}_{2}, \mathfrak{G}_{3}$ and $\mathfrak{G}_{4}$, the last one being the same as Theorem 1.2 above. See Section 2 for a quick overview of the meanings of these intermediate groups. The proof for $\mathfrak{G}_{1}$ is trivial, the proofs for $\mathfrak{G}_{2}$ and $\mathfrak{G}_{3}$ occupy Sections 8 and 9, and the proof for $\mathfrak{G}_{4}$ appears in Section 7.

Section 10 reviews work of Rémy [2002] on the adjoint representation of a Kac-Moody group, regarded as a representation of the corresponding Steinberg group. The definition of $\mathfrak{S t}$ is as the direct limit of a family of unipotent groups, and we use the adjoint representation to show that the natural maps from these groups to $\mathfrak{S t}$ are embeddings. This is necessary for the proof of Theorem 1.1 in Section 11. Finally, in Section 12 we discuss finite presentability of pre-Steinberg groups and Kac-Moody groups. In particular, we prove Theorems 1.4 and 1.5. The result for pre-Steinberg groups relies heavily on work of Splitthoff.

\section{Examples}

In this section we give our presentation of $\mathfrak{P S t}_{A}(R)=\mathfrak{S t}_{A}(R)$ when $R$ is a commutative ring and $A=A_{1}, A_{2}, B_{2}$ or $G_{2}$. It is mostly a writing-out of the general construction in Section 7. Because of the direct limit property of the pre-Steinberg group (Corollary 1.3), understanding these cases, together with

$$
\mathfrak{P S t} \mathfrak{A}_{1}^{2}=\mathfrak{P S t _ { A _ { 1 } }} \times \mathfrak{P S} \mathfrak{t}_{A_{1}},
$$

is enough to present $\mathfrak{P S t}_{A}$ whenever $A$ is 2 -spherical. As usual, we are mainly interested in the presentation when $\mathfrak{P S} \mathfrak{t}$ and $\mathfrak{S t}$ coincide. This happens in any of the cases of Theorem 1.1. 
For generators we take formal symbols $S, S^{\prime}, X(t)$ and $X^{\prime}(t)$, with $t$ varying over $R$. The primed generators should be omitted in the $A_{1}$ case. We divide the relations into batches 0 through 4 , with several intermediate groups having useful descriptions. At the end of the section we give an overview of these descriptions. For now we make only brief remarks. The batch 0 relations make the $S$ 's generate something like the Weyl group. The batch 1 relations make the $X(t)$ 's additive in $t$. The batch 2 relations describe the interaction between the $S$ 's and the $X(t)$ 's. These are the essentially new component of our approach to Steinberg groups. The batch 3 relations are Chevalley relations, describing commutators of conjugates of the $X(t)$ 's by various words in the $S$ 's. Finally, the batch 4 relations are Steinberg's $A_{1}$-specific relations, and relations identifying the $S$ 's with the generators of the "Weyl group" inside the Steinberg group.

In the presentations we write $x \rightleftarrows y$ to indicate that $x$ and $y$ commute. The notation "(\& primed)" next to a relation means to also impose the relation got from it by the typographical substitution $S \leftrightarrow S^{\prime}$ and $X(t) \leftrightarrow X^{\prime}(t)$.

Example 2.1 $\left(A_{1}\right)$. We take generators $S$ and $X(t)$, with $t$ varying over $R$. There are no batch 0 or batch 3 relations:

Batch 1:

Batch 2:

Batch 4:

$$
\begin{aligned}
X(t) X(u) & =X(t+u) \\
S^{2} & \rightleftarrows X(t) \\
S & =\tilde{s}(1) \\
\tilde{h}(r) \cdot X(t) \cdot \tilde{h}(r)^{-1} & =X\left(r^{2} t\right) \\
\tilde{h}(r) \cdot S X(t) S^{-1} \cdot \tilde{h}(r)^{-1} & =S X\left(r^{-2} t\right) S^{-1}
\end{aligned}
$$

These relations hold for all $t, u \in R$ and all $r$ in the unit group $R^{*}$ of $R$, where

$$
\begin{aligned}
\tilde{s}(r) & :=X(r) \cdot S X(1 / r) S^{-1} \cdot X(r), \\
\tilde{h}(r) & :=\tilde{s}(r) \tilde{s}(-1) .
\end{aligned}
$$

This is essentially Steinberg's original presentation (the group $G^{\prime}$ on page 78 of [Steinberg 1968]), with a slightly different generating set.

Example 2.2 $\left(A_{2}\right)$. We take generators $S, S^{\prime}, X(t)$ and $X^{\prime}(t)$, with $t$ varying over $R$ :

Batch 0:

$$
S S^{\prime} S=S^{\prime} S S^{\prime}
$$

$$
S^{2} \cdot S^{\prime} \cdot S^{-2}=S^{-1}
$$

(\& primed)

Batch 1:

$$
X(t) X(u)=X(t+u)
$$

(\& primed)

Batch 2:

$$
S^{2} \rightleftarrows X(t)
$$

(\& primed)

$$
\begin{aligned}
S^{2} \cdot X^{\prime}(t) \cdot S^{-2} & =X^{\prime}(-t) \\
S S^{\prime} X(t) & =X^{\prime}(t) S S^{\prime}
\end{aligned}
$$$$
\text { (\& primed) }
$$

(\& primed) 
Batch 3:

$$
\begin{aligned}
{\left[X(t), X^{\prime}(u)\right] } & =S X^{\prime}(t u) S^{-1} \\
X(t) & \rightleftarrows S X^{\prime}(u) S^{-1}
\end{aligned}
$$

Batch 4:

$$
S=X(1) S X(1) S^{-1} X(1) \quad(\& \text { primed })
$$

As before, these relations hold for all $t, u \in R$. The diagram automorphism is given by $S \leftrightarrow S^{\prime}$ and $X(t) \leftrightarrow X^{\prime}(t)$.

Example 2.3 $\left(B_{2}\right)$. We take generators $S, S^{\prime}, X(t)$ and $X^{\prime}(t)$, with $t$ varying over $R$. Unprimed letters correspond to the short simple root and primed letters to the long one:

Batch 0:

$$
\begin{aligned}
S S^{\prime} S S^{\prime} & =S^{\prime} S S^{\prime} S \\
S^{2} & \rightleftarrows S^{\prime} \\
S^{\prime 2} \cdot S \cdot S^{\prime-2} & =S^{-1}
\end{aligned}
$$

Batch 1:

$$
X(t) X(u)=X(t+u)
$$

Batch 2:

$$
\begin{aligned}
S^{2} & \rightleftarrows X(t) \\
S^{2} & \rightleftarrows X^{\prime}(t) \\
S^{\prime 2} \cdot X(t) \cdot S^{\prime-2} & =X(-t) \\
S S^{\prime} S & \rightleftarrows X^{\prime}(t)
\end{aligned}
$$

(\& primed) (2-22)

Batch 3:

$$
\begin{aligned}
S X^{\prime}(t) S^{-1} & \rightleftarrows S^{\prime} X(u) S^{-1} \\
X^{\prime}(t) & \rightleftarrows S X^{\prime}(u) S^{-1} \\
{\left[X(t), S^{\prime} X(u) S^{\prime-1}\right] } & =S X^{\prime}(-2 t u) S^{-1} \\
{\left[X(t), X^{\prime}(u)\right] } & =S^{\prime} X(-t u) S^{\prime-1} \cdot S X^{\prime}\left(t^{2} u\right) S^{-1}
\end{aligned}
$$

Batch 4:

$$
S=X(1) S X(1) S^{-1} X(1) \quad(\& \text { primed })
$$

Example 2.4 $\left(G_{2}\right)$. We take generators $S, S^{\prime}, X(t)$ and $X^{\prime}(t)$ as in the $B_{2}$ case:

Batch 0:

$$
S S^{\prime} S S^{\prime} S S^{\prime}=S^{\prime} S S^{\prime} S S^{\prime} S
$$

$$
S^{2} \cdot S^{\prime} \cdot S^{-2}=S^{-1}
$$

(\& primed) (2-29)

Batch 1:

$$
X(t) X(u)=X(t+u)
$$

(\& primed)

Batch 2:

$$
S^{2} \rightleftarrows X(t)
$$

(\& primed)

$$
\begin{aligned}
S^{2} \cdot X^{\prime}(t) \cdot S^{-2} & =X^{\prime}(-t) \\
S S^{\prime} S S^{\prime} S & \rightleftarrows X^{\prime}(t)
\end{aligned}
$$$$
\text { (\& primed) }
$$

(\& primed)

Batch 3:

$$
\begin{aligned}
X^{\prime}(t) & \rightleftarrows S^{\prime} S X^{\prime}(u) S^{-1} S^{-1} \\
S S^{\prime} X(t) S^{\prime-1} S^{-1} & \rightleftarrows S^{\prime} S X^{\prime}(u) S^{-1} S^{\prime-1}
\end{aligned}
$$




$$
\begin{aligned}
& S X^{\prime}(t) S^{-1} \rightleftarrows S^{\prime} X(u) S^{\prime-1} \\
& {\left[X^{\prime}(t), S X^{\prime}(u) S^{-1}\right]=S^{\prime} S X^{\prime}(t u) S^{-1} S^{\prime-1} } \\
& {\left[X(t), S S^{\prime} X(u) S^{\prime-1} S^{-1}\right]=S X^{\prime}(3 t u) S^{-1} } \\
& {\left[X(t), S^{\prime} X(u) S^{\prime-1}\right]=S S^{\prime} X(-2 t u) S^{\prime-1} S^{-1} } \\
& \cdot S X^{\prime}\left(-3 t^{2} u\right) S^{-1} \\
& \cdot S^{\prime} S X^{\prime}\left(-3 t u^{2}\right) S^{-1} S^{\prime-1} \\
& {\left[X(t), X^{\prime}(u)\right]=S S^{\prime} X\left(t^{2} u\right) S^{\prime-1} S^{-1} } \\
& \cdot S^{\prime} X(-t u) S^{\prime-1} \cdot S X^{\prime}\left(t^{3} u\right) S^{-1} \\
& \cdot S^{\prime} S X^{\prime}\left(-t^{3} u^{2}\right) S^{-1} S^{\prime-1}
\end{aligned}
$$

Batch 4:

$$
S=X(1) S X(1) S^{-1} X(1)
$$

Now we explain the meaning of the batches. The group with generators $S$ and $S^{\prime}$, modulo the batch 0 relations, is what we call $\widehat{W}$ in Section 7. It is an extension of the Weyl group $W$, slightly "more extended" than a better-known extension of $W$ introduced by Tits [1966a]. We write $W^{*}$ for Tits' extension and discuss it in Section 5. "More extended" means that $\widehat{W} \rightarrow W$ factors through $W^{*}$. The kernel of $W^{*} \rightarrow W$ is an elementary abelian 2-group, while the kernel of $\widehat{W} \rightarrow W$ can be infinite and nilpotent of class 2 . These details are not needed for a general understanding.

The group with generators $X(t)$ and $X^{\prime}(t)$, modulo the batch 1 relations, is what we call $\mathfrak{G}_{1}(R)$ in Section 7. It is just a free product of copies of the additive group of $R$, one for each simple root.

The group generated by $S, S^{\prime}$ and the $X(t)$ and $X^{\prime}(t)$, modulo the relations from batches 0 through 2 , is what we call $\mathfrak{G}_{2}(R)$ in Section 7. It is isomorphic to $\left(*_{\alpha \in \Phi} R\right) \rtimes \widehat{W}$ by Theorem 7.5 , where $\Phi$ is the set of all roots. In fact, this theorem applies to any generalized Cartan matrix $A$. This is the main technical result of the paper, and the batch 2 relations are the main new ingredient in our treatment of the Steinberg groups. Furthermore, Theorem 7.5 generalizes to groups with a root group datum in the sense of [Tits 1992; Caprace and Rémy 2009]; see Remark 7.6. This should lead to generalizations of our results with such groups in place of Kac-Moody groups.

The batch 3 relations are a few of the Chevalley relations, written in a manner due to Demazure; see Section 7 for discussion and references. No batch 3 relations are present in the $A_{1}$ case. In the $A_{2}, B_{2}$ and $G_{2}$ cases, adjoining them yields $\operatorname{St}(R) \rtimes \widehat{W}$, by Theorem 7.11. For any generalized Cartan matrix $A$, the corresponding presentation is called $\mathfrak{G}_{3}(R)$ in Section 7 , and Theorem 7.11 asserts that it is isomorphic to $\mathfrak{P S} \mathfrak{t}^{\text {Tits }}(R) \rtimes \widehat{W}$. Here $\mathfrak{P S} \mathfrak{t}^{\text {Tits }}$ is the "pre-" version of Tits' version of the Steinberg group. See Section 7 for more details. 
Adjoining the batch 4 relations yields the group called $\mathfrak{G}_{4}(R)$ in Section 7. In all four examples this coincides with $\mathfrak{S t}_{A}(R)$. This result is really the concatenation of Theorem 7.12 , that $\mathfrak{G}_{4}$ equals $\mathfrak{P S t}_{A}$ (for any $A$ ), with the isomorphism $\mathfrak{P S t}_{A}=\mathfrak{S t}_{A}$ when $A$ is spherical.

\section{Diagram automorphisms}

In this section we specialize our presentations of $\mathfrak{S t}_{B_{2}}(R)$ and $\mathfrak{S t}_{G_{2}}(R)$ when the ground ring $R$ has characteristic 2 or 3 , respectively. The exceptional diagram automorphisms are then visible. These results are not needed later in the paper.

We begin with the $B_{2}$ case, so assume $2=0$ in $R$. Then $X(t)=X(-t)$ for all $t$. In particular, the right side of (2-27) is its own inverse, so $S$ and $S^{\prime}$ have order 2. The relations involving $S^{2}$ or $S^{\prime 2}$ are therefore trivial and may be omitted. Also, the right side of (2-25) is the identity, so that (2-25) is the primed version of (2-24). In summary, the defining relations for $\mathfrak{S t}$ are now the following, with $t$ and $u$ varying over $R$ :

$$
\begin{aligned}
S S^{\prime} S S^{\prime} & =S^{\prime} S S^{\prime} S \\
X(t) X(u) & =X(t+u) \\
S S^{\prime} S & \rightleftarrows X^{\prime}(t) \\
S X^{\prime}(t) S^{-1} & \rightleftarrows S^{\prime} X(u) S^{\prime-1} \\
X^{\prime}(t) & \rightleftarrows S X^{\prime}(u) S^{-1} \\
{\left[X(t), X^{\prime}(u)\right] } & =S^{\prime} X(-t u) S^{-1} \cdot S X^{\prime}\left(t^{2} u\right) S^{-1} \\
S & =X(1) S X(1) S^{-1} X(1)
\end{aligned}
$$

(\& primed) (3-7)

Theorem 3.1. Suppose $R$ is a ring of characteristic 2. Then the map $S \leftrightarrow S^{\prime}$, $X^{\prime}(t) \mapsto X(t) \mapsto X^{\prime}\left(t^{2}\right)$ extends to an endomorphism $\phi$ of $\mathfrak{S t}_{B_{2}}(R)$. If $R$ is a perfect field then $\phi$ is an automorphism.

Proof. One must check that each relation (3-1)-(3-7) remains true after the substitution $S \leftrightarrow S^{\prime}, X^{\prime}(t) \mapsto X(t) \mapsto X^{\prime}\left(t^{2}\right)$. It is easy to check that every relation maps to its primed form (except that some $t$ 's and $u$ 's are replaced by their squares). The relations (3-1), (3-4) and (3-6) are their own primed forms. Only (3-6) deserves any comment: we must check the identity

$$
\left[X^{\prime}\left(t^{2}\right), X(u)\right]=S X^{\prime}\left(t^{2} u^{2}\right) S^{-1} \cdot S^{\prime} X\left(t^{2} u\right) S^{\prime-1}
$$

in $\mathfrak{S t}$. The left side equals $\left[X(u), X^{\prime}\left(t^{2}\right)\right]^{-1}$. The identity follows by expanding the commutator using (3-6).

Now suppose $R$ is a perfect field. By a similar argument, one can check that there is an endomorphism $\psi$ of $\mathfrak{S t}$ that fixes $S$ and $S^{\prime}$, and for each $t \in R$ sends $X(t)$ 
to $X(\sqrt{t})$ and $X^{\prime}(t)$ to $X^{\prime}(\sqrt{t})$. (Because $R$ is a perfect field of characteristic 2, square roots exist and are unique, and $t \mapsto \sqrt{t}$ is a field automorphism.) Since $\psi \circ \phi \circ \phi$ sends each generator to itself, $\phi$ and $\psi$ must be isomorphisms.

Now we consider the $G_{2}$ case, so suppose $3=0$ in $R$. The main simplifications of Section 2's presentation of $\mathfrak{S t}$ are that the right side of (2-38) is the identity, so (2-38) is the primed version of (2-34), and that the last two terms on the right of (2-39) are trivial, so that (2-39) is the primed version of (2-37). So the relations simplify to:

$$
\begin{array}{rlrl}
S S^{\prime} S S^{\prime} S S^{\prime} & =S^{\prime} S S^{\prime} S S^{\prime} S & & \\
S^{2} \cdot S^{\prime} \cdot S^{-2} & =S^{\prime-1} & & \text { (\& primed) } \\
X(t) X(u) & =X(t+u) & & \text { (\& primed) } \\
S^{2} & \rightleftarrows X(t) & & \text { (\& primed) } \\
S^{2} \cdot X^{\prime}(t) \cdot S^{-2} & =X^{\prime}(-t) & & \text { (\& primed) } \\
S S^{\prime} S S^{\prime} S & \rightleftarrows X^{\prime}(t) & \text { (\& primed) } \\
X^{\prime}(t) & \rightleftarrows S^{\prime} S X^{\prime}(u) S^{-1} S^{-1} & & \text { (\& primed) } \\
S S^{\prime} X(t) S^{\prime-1} S^{-1} & \rightleftarrows S^{\prime} S X^{\prime}(u) S^{-1} S^{-1} & \\
S X^{\prime}(t) S^{-1} & \rightleftarrows S^{\prime} X(u) S^{-1} & & \\
{\left[X^{\prime}(t), S X^{\prime}(u) S^{-1}\right]} & =S^{\prime} S X^{\prime}(t u) S^{-1} S^{-1} & & \\
{\left[X(t), X^{\prime}(u)\right]} & =S S^{\prime} X\left(t^{2} u\right) S^{\prime-1} S^{-1} & & \\
\cdot S^{\prime} X(-t u) S^{\prime-1} \cdot S X^{\prime}\left(t^{3} u\right) S^{-1} & \cdot S^{\prime} S X^{\prime}\left(-t^{3} u^{2}\right) S^{-1} S^{\prime-1} &
\end{array}
$$

The following theorem is proven just like the previous one.

Theorem 3.2. Suppose $R$ is a ring of characteristic 3. Then the map $S \leftrightarrow S^{\prime}$, $X^{\prime}(t) \mapsto X(t) \mapsto X^{\prime}\left(t^{3}\right)$ extends to an endomorphism $\phi$ of $\mathfrak{S t}_{G_{2}}(R)$. If $R$ is a perfect field then $\phi$ is an automorphism.

The exceptional diagram automorphisms lead to the famous Suzuki and Ree groups. If $R$ is the finite field $\mathbb{F}_{q}$ where $q=2^{\text {odd }}$, then the Frobenius automorphism of $R$ (namely squaring) is the square of a field automorphism $\xi$. Writing $\xi$ also for the induced automorphism of $\mathfrak{S t}_{B_{2}}(R)$, the Suzuki group is defined as the subgroup where $\xi$ agrees with $\phi$. The same construction with $F_{4}$ in place of $B_{2}$ yields the large Ree groups, and in characteristic 3 with $G_{2}$ yields the small Ree groups. These groups are "like" groups of Lie type in that they admit root group data in the sense of [Tits 1992] or [Caprace and Rémy 2009], but they are not algebraic groups. 
Hée generalized this [2008]. He showed that when a group with a root group datum admits two automorphisms that permute the simple roots' root groups, and satisfy some other natural conditions, then the subgroup where they coincide also admits a root group datum. Furthermore, the Weyl group for the subgroup may be computed in a simple way from the Weyl group for the containing group. For example, over $\mathbb{F}_{q}$ with $q=2^{\text {odd }}$, the Kac-Moody group

contains a Kac-Moody-like analogue of the Suzuki groups. By Hée's theorem, its Weyl group is

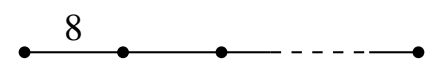

Hée [1990] constructs diagram automorphisms in a different way than we do, and discusses the case " $G_{4}$ " in some detail.

\section{The Kac-Moody algebra}

In this section we begin the technical part of the paper, by recalling the Kac-Moody algebra and some notation from [Tits 1987]. All group actions are on the left. We will use the following general notation:

$\langle$,$\rangle a bilinear pairing$

$\langle\cdot\rangle$ group generated by the elements enclosed

$\langle\cdot \mid \cdot\rangle$ a group presentation

$[x, y] \quad x y x^{-1} y^{-1}$ if $x$ and $y$ are group elements

$*$ free product of groups (possibly with amalgamation)

The Steinberg group is built from a generalized Cartan matrix $A$, for which we will use the following notation:

$I$ an index set (the nodes of the Dynkin diagram)

$i, j \quad$ will always indicate elements of $I$

$A=\left(A_{i j}\right)$ a generalized Cartan matrix: an integer matrix satisfying $A_{i i}=2$, $A_{i j} \leq 0$ if $i \neq j$, and $A_{i j}=0 \Longleftrightarrow A_{j i}=0$

$m_{i j} \quad$ numerical edge labels of the Dynkin diagram: $m_{i j}=2,3,4,6$ or $\infty$, according to whether $A_{i j} A_{j i}=0,1,2,3$ or $\geq 4$, except that $m_{i i}=1$

$W$ the Coxeter group $\left\langle s_{i \in I}\right|\left(s_{i} s_{j}\right)^{m_{i j}}=1$ if $\left.m_{i j} \neq \infty\right\rangle$

$\mathbb{Z}^{I}$ the free abelian group with basis $\alpha_{i \in I}$, called the simple roots; $W$ acts on $\mathbb{Z}^{I}$ by $s_{i}\left(\alpha_{j}\right)=\alpha_{j}-A_{i j} \alpha_{i}$ (this action is faithful by the theory of the Tits cone [Bourbaki 2002, Chapter V, §4.4])

$\Phi$ the set of (real) roots: all $w \alpha_{i}$ with $w \in W$ and $i \in I$ 
The Kac-Moody algebra $\mathfrak{g}=\mathfrak{g}_{A}$ associated to $A$ means the complex Lie algebra with generators $e_{i \in I}, f_{i \in I}, \bar{h}_{i \in I}$ and defining relations

$$
\left[\bar{h}_{i}, e_{j}\right]=A_{i j} e_{j}, \quad\left[\bar{h}_{i}, f_{i}\right]=-A_{i j} f_{j}, \quad\left[\bar{h}_{i}, \bar{h}_{j}\right]=0, \quad\left[e_{i}, f_{i}\right]=-\bar{h}_{i},
$$

and, for $i \neq j$,

$$
\left[e_{i}, f_{j}\right]=0, \quad\left(\operatorname{ad} e_{i}\right)^{1-A_{i j}}\left(e_{j}\right)=\left(\operatorname{ad} f_{i}\right)^{1-A_{i j}}\left(f_{j}\right)=0 .
$$

(Note: $(\operatorname{ad} x)(y)$ means $[x, y]$. Also, Tits' generators differ from Kac's generators [1990] by a sign on $f_{i}$.) For any $i$ the linear span of $e_{i}, f_{i}$ and $\bar{h}_{i}$ is isomorphic to $\mathfrak{s l}_{2} \mathbb{C}$, via

$$
e_{i}=\left(\begin{array}{ll}
0 & 1 \\
0 & 0
\end{array}\right), \quad f_{i}=\left(\begin{array}{rr}
0 & 0 \\
-1 & 0
\end{array}\right), \quad \bar{h}_{i}=\left(\begin{array}{rr}
1 & 0 \\
0 & -1
\end{array}\right)
$$

We equip $\mathfrak{g}$ with a grading by $\mathbb{Z}^{I}$, with $\bar{h}_{i} \in \mathfrak{g}_{0}, e_{i} \in \mathfrak{g}_{\alpha_{i}}$ and $f_{i} \in \mathfrak{g}_{-\alpha_{i}}$. For $\alpha \in \mathbb{Z}^{I}$ we refer to $\mathfrak{g}_{\alpha}$ as its root space, and abbreviate $\mathfrak{g}_{\alpha_{i}}$ to $\mathfrak{g}_{i}$. We follow [Tits 1987] in saying "root" for "real root" (meaning an element of $\Phi$ ). Imaginary roots play no role in this paper.

\section{The extension $W^{*} \subseteq$ Aut $\mathfrak{g}$ of the Weyl group}

The Weyl group $W$ does not necessarily act on $\mathfrak{g}$, but a certain extension of it called $W^{*}$ does. In this section we review its basic properties. The results through Theorem 5.5 are due to Tits. The last result is new: it describes the root stabilizers in $W^{*}$. The proof relies on Brink's study [1996] of reflection centralizers in Coxeter groups, in the form given in [Allcock 2013].

It is standard [Kac 1990, Lemma 3.5] that ad $e_{i}$ and ad $f_{i}$ are locally nilpotent on $\mathfrak{g}$, so their exponentials are automorphisms of $\mathfrak{g}$. Furthermore,

$$
\left(\exp \operatorname{ad} e_{i}\right)\left(\exp \operatorname{ad} f_{i}\right)\left(\exp \operatorname{ad} e_{i}\right)=\left(\exp \operatorname{ad} f_{i}\right)\left(\exp \operatorname{ad} e_{i}\right)\left(\exp \operatorname{ad} f_{i}\right) .
$$

We write $s_{i}^{*}$ for this element of Aut $\mathfrak{g}$ and $W^{*}$ for $\left\langle s_{i \in I}^{*}\right\rangle \subseteq$ Aut $\mathfrak{g}$. One shows [Kac 1990, Lemma 3.8] that $s_{i}^{*}\left(\mathfrak{g}_{\alpha}\right)=\mathfrak{g}_{s_{i}(\alpha)}$ for all $\alpha \in \mathbb{Z}^{I}$. This defines a $W^{*}$-action on $\mathbb{Z}^{I}$, with $s_{i}^{*}$ acting as $s_{i}$. Since $W$ acts faithfully on $\mathbb{Z}^{I}$ this yields a homomorphism $W^{*} \rightarrow W$. Using $W^{*}$, the general theory [Kac 1990, Proposition 5.1] shows that $\mathfrak{g}_{\alpha}$ is 1-dimensional for any $\alpha \in \Phi$.

Let $\mathbb{Z}^{I \vee}$ be the free abelian group with basis the formal symbols $\alpha_{i \in I}^{\vee}$ and define a bilinear pairing $\mathbb{Z}^{I \vee} \times \mathbb{Z}^{I} \rightarrow \mathbb{Z}$ by $\left\langle\alpha_{i}^{\vee}, \alpha_{j}\right\rangle=A_{i j}$. We define an action of $W$ on $\mathbb{Z}^{I \vee}$ by $s_{i}\left(\alpha_{j}^{\vee}\right)=\alpha_{j}^{\vee}-A_{j i} \alpha_{i}^{\vee}$. One can check that this action satisfies $\left\langle w \alpha^{\vee}, w \beta\right\rangle=\left\langle\alpha^{\vee}, \beta\right\rangle$. There is a homomorphism $\operatorname{Ad}: \mathbb{Z}^{I \vee} \rightarrow$ Aut $\mathfrak{g}$, with $\operatorname{Ad}\left(\alpha^{\vee}\right)$ acting on $\mathfrak{g}_{\beta}$ by $(-1)^{\left\langle\alpha^{\vee}, \beta\right\rangle}$, where $\beta \in \mathbb{Z}^{I}$. The proof of the next lemma is easy and standard. 
Lemma 5.1. $\mathrm{Ad}: \mathbb{Z}^{I \vee} \rightarrow$ Aut $\mathfrak{g}$ is $W^{*}$-equivariant in the sense that

$$
w^{*} \cdot \operatorname{Ad}\left(\alpha^{\vee}\right) \cdot w^{*-1}=\operatorname{Ad}\left(w \alpha^{\vee}\right),
$$

where $\alpha^{\vee} \in \mathbb{Z}^{I \vee}$ and $w$ is the image in $W$ of $w^{*} \in W^{*}$.

Lemma 5.2. The following identities hold in Aut g:

(i) $s_{i}^{* 2}=\operatorname{Ad}\left(\alpha_{i}^{\vee}\right)$.

(ii) $s_{i}^{*}\left(s_{j}^{*}\right)^{2} s_{i}^{*-1}=\left(s_{j}^{*}\right)^{2}\left(s_{i}^{*}\right)^{-2 A_{j i}}$.

Proof sketch. (i) Identifying the span of $e_{i}, f_{i}, \bar{h}_{i}$ with $\mathfrak{s l}_{2} \mathbb{C}$ as in (4-1) identifies $s_{i}^{* 2}$ with $\left(\begin{array}{rr}-1 & 0 \\ 0 & -1\end{array}\right) \in \mathrm{SL}_{2} \mathbb{C}$. One uses the representation theory of $\mathrm{SL}_{2} \mathbb{C}$ to see how this acts on $\mathfrak{g}$ 's weight spaces.

(ii) Use (i) to identify $s_{j}^{* 2}$ with $\operatorname{Ad}\left(\alpha_{j}^{\vee}\right)$, then Lemma 5.1 to identify $s_{i}^{*} \operatorname{Ad}\left(\alpha_{j}^{\vee}\right) s_{i}^{*-1}$ with $\operatorname{Ad}\left(s_{i}\left(\alpha_{j}^{\vee}\right)\right)$, then the formula defining $s_{i}\left(\alpha_{j}^{\vee}\right)$, and finally (i) again to convert back to $s_{i}^{* 2}$ and $s_{j}^{* 2}$.

To understand the relations satisfied by the $s_{i}^{*}$ it will be useful to have a characterization of them in terms of the choice of $e_{i}$ (together with the grading on $\mathfrak{g}$ ). This is part of Tits' "trijection" [1966b, §1.1]. In the notation of the next lemma, $s_{i}^{*}$ is $s_{e_{i}}^{*}$ (or equally well $s_{f_{i}}^{*}$ ).

Lemma 5.3. If $\alpha \in \Phi$ and $e \in \mathfrak{g}_{\alpha}-\{0\}$ then there exists a unique $f \in \mathfrak{g}_{-\alpha}$ such that

$$
s_{e}^{*}:=(\exp \operatorname{ad} e)(\exp \operatorname{ad} f)(\exp \operatorname{ad} e)
$$

exchanges $\mathfrak{g}_{ \pm \alpha}$. Furthermore, $s_{e}^{*}$ coincides with $s_{f}^{*}$ and exchanges $e$ and $f$. Finally, if $\phi \in$ Aut $\mathfrak{g}$ permutes the $\mathfrak{g}_{\beta \in \Phi}$ then $\phi s_{e}^{*} \phi^{-1}=s_{\phi(e)}^{*}$.

Lemma 5.4. (i) If $m_{i j}=3$ then $s_{j}^{*} s_{i}^{*}\left(e_{j}\right)=e_{i}$.

(ii) If $m_{i j}=2,4$ or 6 then $e_{j}$ is fixed by $s_{i}^{*}, s_{i}^{*} s_{j}^{*} s_{i}^{*}$ or $s_{i}^{*} s_{j}^{*} s_{i}^{*} s_{j}^{*} s_{i}^{*}$, respectively.

Proof. Part (i) follows from direct calculation in $\mathfrak{s l}_{3} \mathbb{C}$. In the $m_{i j}=2$ case of (ii) we have $\left(\operatorname{ad} e_{i}\right)\left(e_{j}\right)=\left(\operatorname{ad} f_{i}\right)\left(e_{j}\right)=0$, and $s_{i}^{*}\left(e_{j}\right)=e_{j}$ follows immediately. The remaining cases involve careful tracking of signs. We will write $\left(\mathfrak{s l}_{2} \mathbb{C}\right)_{i}$ for the span of $e_{i}, f_{i}, \bar{h}_{i}$.

If $m_{i j}=4$ then $\left\{A_{i j}, A_{j i}\right\}=\{-1,-2\}$ and $\alpha_{i}$ and $\alpha_{j}$ are simple roots for a $B_{2}$ root system. Using Lemma 5.3,

$$
s_{i}^{*} s_{j}^{*} s_{i}^{*}\left(e_{j}\right)=s_{i}^{*} s_{e_{j}}^{*} s_{i}^{*-1} s_{i}^{* 2}\left(e_{j}\right)=s_{s_{i}^{*}\left(e_{j}\right)}^{*}\left(\left(\operatorname{Ad} \alpha_{i}^{\vee}\right)\left(e_{j}\right)\right)=(-1)^{A_{i j}} s_{s_{i}^{*}\left(e_{j}\right)}^{*}\left(e_{j}\right) .
$$

Suppose first that $A_{i j}=-2$. Then $\alpha_{i}$ is the short simple root, $\alpha_{j}$ the long one, and $s_{i}\left(\alpha_{j}\right)$ is a long root orthogonal to $\alpha_{j}$. We have

$$
s_{s_{i}^{*}\left(e_{j}\right)}^{*}=\left(\exp \operatorname{ad} s_{i}^{*}\left(e_{j}\right)\right)\left(\exp \operatorname{ad} s_{i}^{*}\left(f_{j}\right)\right)\left(\exp \operatorname{ad} s_{i}^{*}\left(e_{j}\right)\right) \in \exp \operatorname{ad}\left(s_{i}^{*}\left(\left(\mathfrak{s l}_{2} \mathbb{C}\right)_{j}\right)\right) .
$$


Now, $s_{i}^{*}\left(\left(\mathfrak{s l}_{2} \mathbb{C}\right)_{j}\right)$ annihilates $\mathfrak{g}_{j}$ because its root string through $\alpha_{j}$ has length 1 . So $s_{s_{i}^{*}\left(e_{j}\right)}^{*}$ fixes $e_{j}$ and (5-2) becomes

$$
s_{i}^{*} s_{j}^{*} s_{i}^{*}\left(e_{j}\right)=(-1)^{A_{i j}} e_{j}=(-1)^{-2} e_{j}=e_{j} .
$$

On the other hand, if $A_{i j}=-1$ then $\alpha_{j}$ and $s_{i}\left(\alpha_{j}\right)$ are orthogonal short roots. Now the root string through $\alpha_{j}$ for $s_{i}^{*}\left(\left(\mathfrak{s l}_{2} \mathbb{C}\right)_{j}\right)$ has length 3 , so the $s_{i}^{*}\left(\left(\mathfrak{s l}_{2} \mathbb{C}\right)_{j}\right)$-module generated by $e_{j}$ is a copy of the adjoint representation. In particular,

$$
s_{s_{i}^{*}\left(e_{j}\right)}^{*}=s_{i}^{*} s_{j}^{*} s_{i}^{*-1}
$$

acts on $\mathfrak{g}_{j}$ by the same scalar as on the Cartan subalgebra $s_{i}^{*}\left(\mathbb{C} \bar{h}_{j}\right)$ of $s_{i}^{*}\left(\left(\mathfrak{s l}_{2} \mathbb{C}\right)_{j}\right)$. This is the same scalar by which $s_{j}^{*}$ acts on $\mathbb{C} \bar{h}_{j}$, which is -1 . So $s_{s_{i}^{*}\left(e_{j}\right)}^{*}$ negates $e_{j}$ and (5-2) reads

$$
s_{i}^{*} s_{j}^{*} s_{i}^{*}\left(e_{j}\right)=(-1)^{A_{i j}}\left(-e_{j}\right)=(-1)^{-1}\left(-e_{j}\right)=e_{j} .
$$

Now suppose $m_{i j}=6$, so that $\left\{A_{i j}, A_{j i}\right\}=\{-1,-3\}, \alpha_{i}$ and $\alpha_{j}$ are simple roots for a $G_{2}$ root system, and $s_{i} s_{j}\left(\alpha_{i}\right) \perp \alpha_{j}$. Then

$$
\begin{aligned}
s_{i}^{*} s_{j}^{*} s_{i}^{*} s_{j}^{*} s_{i}^{*}\left(e_{j}\right) & =\left(s_{i}^{*} s_{j}^{*} s_{e_{i}}^{*} s_{j}^{*-1} s_{i}^{*-1}\right) s_{i}^{*} s_{j}^{*} s_{j}^{*} s_{i}^{*}\left(e_{j}\right) \\
& =s_{s_{i}^{*}}^{*} s_{j}^{*}\left(e_{i}\right) \circ\left(s_{i}^{*} s_{j}^{* 2} s_{i}^{*-1}\right) \circ s_{i}^{* 2}\left(e_{j}\right) \\
& =s_{s_{i}^{*}}^{*} s_{j}^{*}\left(e_{i}\right) \circ s_{j}^{* 2} s_{i}^{*-2 A_{j i}} \circ s_{i}^{* 2}\left(e_{j}\right) \\
& =s_{s_{i}^{*}}^{*} s_{j}^{*}\left(e_{i}\right) \circ s_{j}^{* 2} s_{i}^{* 4 \text { or } 8}\left(e_{j}\right) \\
& =s_{s_{i}^{*} s_{j}^{*}\left(e_{i}\right)}\left(e_{j}\right) .
\end{aligned}
$$

The root string through $\alpha_{j}$ for $s_{i}^{*} s_{j}^{*}\left(\left(\mathfrak{s l}_{2} \mathbb{C}\right)_{i}\right)$ has length 1 , so arguing as in the $B_{2}$ case shows that $s_{s_{i}^{*} s_{j}^{*}\left(e_{i}\right)}^{*}$ fixes $e_{j}$.

Theorem 5.5 [Tits 1966a, §4.6]. The $s_{i}^{*}$ satisfy the Artin relations of M. That is, if $m_{i j} \neq \infty$ then $s_{i}^{*} s_{j}^{*} \cdots=s_{j}^{*} s_{i}^{*} \cdots$, where there are $m_{i j}$ factors on each side, alternately $s_{i}^{*}$ and $s_{j}^{*}$.

Proof. For $m_{i j}=3$ we start with $e_{j}=s_{i}^{*} s_{j}^{*}\left(e_{i}\right)$ from Lemma 5.4(i). Using Lemma 5.3 yields

$$
s_{j}^{*}=s_{e_{j}}^{*}=s_{s_{i}^{*}}^{*} s_{j}^{*}\left(e_{i}\right)=s_{i}^{*} s_{j}^{*} s_{e_{i}}^{*} s_{j}^{*-1} s_{i}^{*-1}=s_{i}^{*} s_{j}^{*} s_{i}^{*} s_{j}^{*-1} s_{i}^{*-1} .
$$

The other cases are the same.

We will need to understand the $W^{*}$-stabilizer of a simple root $\alpha_{i}$ and how it acts on $\mathfrak{g}_{i}$. The first step is to quote from [Allcock 2013] a refinement of a theorem of Brink [1996] on reflection centralizers in Coxeter groups. Then we will "lift" this result to $W^{*}$ by keeping track of signs. 
Both theorems refer to the "odd Dynkin diagram" $\Delta^{\text {odd }}$, which means the graph with vertex set $I$, where vertices $i$ and $j$ are joined just if $m_{i j}=3$. For $\gamma$ an edge path in $\Delta^{\text {odd }}$, with $i_{0}, \ldots, i_{n}$ the vertices along it, we define

$$
p_{\gamma}:=\left(s_{i_{n-1}} s_{i_{n}}\right)\left(s_{i_{n-2}} s_{i_{n-1}}\right) \cdots\left(s_{i_{1}} s_{i_{2}}\right)\left(s_{i_{0}} s_{i_{1}}\right) .
$$

(If $\gamma$ has length 0 then we set $p_{\gamma}=1$.) For $i \in I$ we write $\Delta_{i}^{\text {odd }}$ for its component of $\Delta^{\text {odd }}$.

Theorem 5.6 [Allcock 2013, Corollary 8]. Suppose $i \in I, Z$ is a set of closed edge paths based at $i$ that generate $\pi_{1}\left(\Delta_{i}^{\text {odd }}, i\right)$, and $\delta_{j}$ is an edge path in $\Delta_{i}^{\text {odd }}$ from $i$ to $j$, for each vertex $j$ of $\Delta_{i}^{\text {odd }}$. For each such $j$ and each $k \in I$ with $m_{j k}$ finite and even, define

$$
r_{j k}:=p_{\delta_{j}}^{-1} \cdot\left\{\begin{array}{c}
s_{k} \\
s_{k} s_{j} s_{k} \\
s_{k} s_{j} s_{k} s_{j} s_{k}
\end{array}\right\} \cdot p_{\delta_{j}}
$$

according to whether $m_{j k}=2,4$ or 6 . Then the $W$-stabilizer of the simple root $\alpha_{i}$ is generated by the $r_{j k}$ and the $p_{z \in Z \text {. }}$

It is easy to see that the $r_{j k}$ and $p_{z}$ stabilize $\alpha_{i}$. In fact, this is the "image under $W^{*} \rightarrow W$ " of the corresponding part of the next theorem.

Theorem 5.7. Suppose $i, Z$ and the $\delta_{j}$ are as in Theorem 5.6. Define $p_{\gamma}^{*}$ and $r_{j k}^{*}$ by attaching $*$ to each $s, p$ and $r$ in (5-3) and (5-4). Then the $p_{z \in Z}^{*}$ and $r_{j k}^{*}$ fix $e_{i}$, and together with the $s_{l \in I}^{* 2}$ they generate the $W^{*}$-stabilizer of $\alpha_{i}$. (By Lemma 5.2(i), $s_{l}^{* 2}$ acts on $e_{i}$ by $\left.(-1)^{A_{l i}}\right)$.

Proof. The $W^{*}$-stabilizer of $\alpha_{i}$ is generated by $\operatorname{ker}\left(W^{*} \rightarrow W\right)$ and any set of elements of $W^{*}$ whose projections to $W$ generate the $W$-stabilizer of $\alpha_{i}$. Now, the $s_{i}^{* 2}$ normally generate the kernel because of the Artin relations. Lemma 5.2(ii) shows that the subgroup they generate is normal, hence equal to this kernel. Since the $p^{*}$ 's and $r^{*}$ 's project to the $p$ 's and $r$ 's of Theorem 5.6, our generation claim follows from that theorem. To see that the $p_{z}^{*}$ 's fix $e_{i}$, apply Lemma 5.4(i) repeatedly. The same argument proves $p_{\delta_{j}}^{*}\left(e_{i}\right)=e_{j}$. Then using Lemma 5.4(ii) shows that $e_{j}$ is fixed by $s_{k}^{*}, s_{k}^{*} s_{j}^{*} s_{k}^{*}$ or $s_{k}^{*} s_{j}^{*} s_{k}^{*} s_{j}^{*} s_{k}^{*}$ according to whether $m_{j k}$ is 2, 4 or 6. Applying $p_{\delta_{j}}^{*-1}$ sends $e_{j}$ back to $e_{i}$, proving $r_{j k}^{*}\left(e_{i}\right)=e_{i}$.

\section{The Steinberg group $\mathfrak{S t}$}

In this section we give an overview of the Steinberg group $\mathfrak{S t}_{A}$, as defined by Tits [1987] and refined by Morita and Rehmann [1990]. The purpose is to be able to compare the pre-Steinberg group $\mathfrak{P S \mathfrak { t } _ { A }}$ (see Section 7) with $\mathfrak{S t}_{A}$. For example, Theorem 1.1 gives many cases in which the natural map $\mathfrak{P S t}_{A}(R) \rightarrow \mathfrak{S t}_{A}(R)$ is an isomorphism. 
The Morita-Rehmann definition is got from Tits' definition by imposing some additional relations. These are also due to Tits, but he imposed them only later in his construction, when defining Kac-Moody groups in terms of $\mathfrak{S t}_{A}$. In the few places where we need to distinguish between the definitions, we will write $\mathfrak{S t}_{A}^{\text {Tits }}$ for Tits' version and $\mathfrak{S t}_{A}$ for the Morita-Rehmann version. In the rest of this section we will regard $A$ as fixed and omit it from the subscripts.

$\mathfrak{A} \mathfrak{d} \mathfrak{d}$ denotes the additive group, regarded as a group scheme over $\mathbb{Z}$. That is, it is the functor assigning to each commutative ring $R$ its underlying abelian group. The Lie algebra of $\mathfrak{A} \mathfrak{d} \mathfrak{d}$ is canonically isomorphic to $\mathbb{Z}$.

For each $\alpha \in \Phi, \mathfrak{g}_{\alpha} \cap W^{*}\left(\left\{e_{i \in I}\right\}\right)$ consists of either one vector or two antipodal vectors. This is [Tits 1987, (3.3.2)] and its following paragraph, which relies on [Tits 1974, §13.31]. Alternately, it follows from our Theorem 5.7. We write $\mathfrak{g}_{\alpha, \mathbb{Z}}$ for the $\mathbb{Z}$-span in $\mathfrak{g}_{\alpha}$ of this element or antipodal pair, and $E_{\alpha}$ for the set of its generators (a set of size 2). The symbol $e$ will always indicate an element of some $E_{\alpha}$. We define $\mathfrak{U}_{\alpha}$ as the group scheme over $\mathbb{Z}$ which is isomorphic to $\mathfrak{A} \mathfrak{d} d$ and has Lie algebra $\mathfrak{g}_{\alpha, \mathbb{Z}}$. That is, $\mathfrak{U}_{\alpha}$ is the functor assigning to each commutative ring $R$ the abelian group $\mathfrak{g}_{\alpha, \mathbb{Z}} \otimes R \cong R$. For $i \in I$ we abbreviate $\mathfrak{U}_{ \pm \alpha_{i}}$ to $\mathfrak{U}_{ \pm i}$.

If $\alpha \in \Phi$ and $e \in E_{\alpha}$ then we define $\mathfrak{x}_{e}$ as the isomorphism $\mathfrak{A d d} \rightarrow \mathfrak{U}_{\alpha}$, whose corresponding Lie algebra isomorphism identifies $1 \in \mathbb{Z}$ with $e \in \mathfrak{g}_{\alpha, \mathbb{Z}}$. For fixed $R$ this amounts to

$$
\mathfrak{x}_{e}(t):=e \otimes t \in \mathfrak{g}_{\alpha, \mathbb{Z}} \otimes R=\mathfrak{U}_{\alpha} .
$$

If $R=\mathbb{R}$ or $\mathbb{C}$ then one may think of $\mathfrak{x}_{e}(t)$ as $\exp (t e)$. For $i \in I$ we abbreviate $\mathfrak{x}_{e_{i}}$ to $\mathfrak{x}_{i}$ and $\mathfrak{x}_{f_{i}}$ to $\mathfrak{x}_{-i}$.

Tits calls a set of roots $\Psi \subseteq \Phi$ prenilpotent if some chamber in the open Tits cone lies on the positive side of all their mirrors and some other chamber lies on the negative side of all of them. (Equivalently, some element of $W$ sends $\Psi$ into the set of positive roots and some other element of $W$ sends $\Psi$ into the set of negative roots.) It follows that $\Psi$ is finite. If $\Psi$ is also closed under addition then it is called nilpotent. In this case $\mathfrak{g}_{\Psi}:=\bigoplus_{\alpha \in \Psi} \mathfrak{g}_{\alpha}$ is a nilpotent Lie algebra [Tits 1987, p. 547].

Lemma 6.1 [Tits 1987, §3.4]. If $\Psi \subseteq \Phi$ is a nilpotent set of roots, then there is a unique unipotent group scheme $\mathfrak{U}_{\Psi}$ over $\mathbb{Z}$ with these properties:

(i) $\mathfrak{U}_{\Psi}$ contains all the $\mathfrak{U}_{\alpha \in \Psi}$.

(ii) $\mathfrak{U}_{\Psi}(\mathbb{C})$ has Lie algebra $\mathfrak{g}_{\Psi}$.

(iii) For any ordering on $\Psi$, the product morphism $\prod_{\alpha \in \Psi} \mathfrak{U}_{\alpha} \rightarrow \mathfrak{U}_{\Psi}$ is an isomorphism of the underlying schemes.

Tits' version $\mathfrak{S t}^{\text {Tits }}$ of the Steinberg group functor is defined as follows. For each prenilpotent pair $\alpha, \beta$ of roots, $\theta(\alpha, \beta)$ is defined as $(\mathbb{N} \alpha+\mathbb{N} \beta) \cap \Phi$ where $\mathbb{N}=$ $\{0,1,2, \ldots\}$. Consider the groups $\mathfrak{U}_{\theta(\alpha, \beta)}$ with $\{\alpha, \beta\}$ varying over all prenilpotent 
pairs. If $\gamma \in \theta(\alpha, \beta)$ then there is a natural injection $\mathfrak{U}_{\gamma} \rightarrow \mathfrak{U}_{\theta(\alpha, \beta)}$, yielding a diagram of inclusions of group functors. $\mathfrak{S t}^{\text {Tits }}$ is defined as the direct limit of this diagram. Every automorphism of $\mathfrak{g}$ that permutes the subgroups $\mathfrak{g}_{\alpha, \mathbb{Z}}$ induces an automorphism of the diagram of inclusions of group functors, hence an automorphism of $\mathfrak{S t}^{\text {Tits }}$. In particular, $W^{*}$ acts on $\mathfrak{S t}^{\text {Tits }}$.

As Tits points out, a helpful but less canonical way to think about $\mathfrak{S t}^{\text {Tits }}(R)$ is to begin with the free product $*_{\alpha \in \Phi} \mathfrak{U}_{\alpha}(R)$ and impose relations of the form

$$
\left[\mathfrak{x}_{e_{\alpha}}(t), \mathfrak{x}_{e_{\beta}}(u)\right]=\prod_{\gamma=m \alpha+n \beta} \mathfrak{x}_{e_{\gamma}}\left(C_{\alpha \beta \gamma} t^{m} u^{n}\right)
$$

for each prenilpotent pair $\alpha, \beta \in \Phi$. Here $\gamma=m \alpha+n \beta$ runs over $\theta(\alpha, \beta)-\{\alpha, \beta\}$, so in particular $m$ and $n$ are positive integers. Also, $e_{\alpha}, e_{\beta}$ and the various $e_{\gamma}$ lie in $E_{\alpha}, E_{\beta}$ and the various $E_{\gamma}$, and must be chosen before the relation can be written down explicitly. The $C_{\alpha \beta \gamma}$ are integers that depend on the position of $\gamma$ relative to $\alpha$ and $\beta$, the choices of $e_{\alpha}, e_{\beta}$ and the $e_{\gamma}$, and the ordering of the product; compare (3) of [Tits 1987]. Usually (6-1) is called "the Chevalley relation of $\alpha$ and $\beta$ ". It is really a family of relations parametrized by $t$ and $u$, and (strictly speaking) not defined without the various choices being fixed.

Unfortunately, Tits' version of the Steinberg group is different from Steinberg's original group when the Dynkin diagram has $A_{1}$ components. Therefore, we follow [Morita and Rehmann 1990] in defining the Steinberg group functor $\mathfrak{S t}$. That is, we impose the additional relations (6-5), which correspond to the relations $\left(\mathrm{B}^{\prime}\right)$ in [Steinberg 1968] or [Morita and Rehmann 1990]. These relations make the "maximal torus" and "Weyl group" act on the root groups $\mathfrak{U}_{\alpha}$ in the expected manner. If $A$ is 2 -spherical without $A_{1}$ components then the Morita-Rehmann relations already hold in $\mathfrak{S t}^{\text {Tits }}$ and this part of the construction can be skipped, by [Tits 1987, $\left(\mathrm{a}_{4}\right)$, p. 550].

The relators involve the following elements of $\mathfrak{S t}^{\text {Tits }}$. If $\alpha \in \Phi$ and $e \in E_{\alpha}$ then recall from Lemma 5.3 that there is a distinguished $f \in E_{-\alpha}$. As the notation suggests, if $e=e_{i}$ then $f=f_{i}$. For any $r \in R^{*}$ we define

$$
\begin{aligned}
\tilde{s}_{e}(r) & :=\mathfrak{x}_{e}(r) \mathfrak{x}_{f}(1 / r) \mathfrak{x}_{e}(r), \\
\tilde{h}_{e}(r) & :=\tilde{s}_{e}(r) \tilde{s}_{e}(-1) .
\end{aligned}
$$

We abbreviate special cases in the usual way: $\tilde{h}_{ \pm i}(r)$ for $\tilde{h}_{e_{i}}(r)$ and $\tilde{h}_{f_{i}}(r), \tilde{s}_{ \pm i}(r)$ for $\tilde{s}_{e_{i}}(r)$ and $\tilde{s}_{f_{i}}(r), \tilde{s}_{ \pm i}$ for $\tilde{s}_{ \pm i}(1)$, and $\tilde{s}_{e}$ for $\tilde{s}_{e}(1)$. It is useful to note several immediate consequences of the definitions: $\tilde{s}_{e}(-r)=\tilde{s}_{e}(r)^{-1}, \tilde{h}_{e}(1)=1$, and

$$
\tilde{s}_{e}(r) \tilde{s}_{e}\left(r^{\prime}\right)^{-1}=\tilde{h}_{e}(r) \tilde{h}_{e}\left(r^{\prime}\right)^{-1} .
$$


Conceptually, the relations we will impose on $\mathfrak{S t}^{\text {Tits }}$ to get $\mathfrak{S t}$ force the conjugation maps of the various $\tilde{s}_{e}(r)$ to be the same as certain automorphisms of $\mathfrak{S t}^{\text {Tits }}$. So we will describe these automorphisms and then state the relations.

Recall from Lemma 5.1 and its preceding remarks that $\mathbb{Z}^{I \vee}$ is the free abelian group generated by formal symbols $\alpha_{i \in I}^{\vee}$. Also, the bilinear pairing $\mathbb{Z}^{I \vee} \times \mathbb{Z}^{I} \rightarrow \mathbb{Z}$ given by $\left\langle\alpha_{i}^{\vee}, \alpha_{j}\right\rangle=A_{i j}$ is $W$-invariant. We defined a map Ad $: \mathbb{Z}^{I \vee} \rightarrow$ Aut $\mathfrak{g}$, which we generalize to $\operatorname{Ad}:\left(R^{*} \otimes \mathbb{Z}^{I \vee}\right) \rightarrow \operatorname{Aut}\left(*_{\alpha \in \Phi} \mathfrak{U}_{\alpha}\right)$ as follows. For any $\alpha^{\vee} \in \mathbb{Z}^{I \vee}, r \in R^{*}$ and $\beta \in \Phi, \operatorname{Ad}\left(r \otimes \alpha^{\vee}\right)$ acts on $\mathfrak{U}_{\beta} \cong R$ by multiplication by

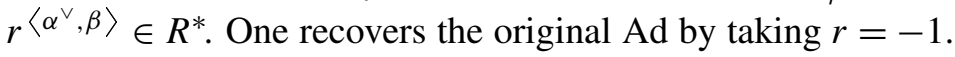

The Chevalley relations have a homogeneity property, namely that $\operatorname{Ad}\left(r \otimes \alpha^{\vee}\right)$ permutes them. This is most visible when they are stated in the form (6-1). Therefore, the action Ad of $R^{*} \otimes \mathbb{Z}^{I \vee}$ on $*_{\alpha \in \Phi} \mathfrak{U}_{\alpha}$ descends to an action on $\mathfrak{S t}^{\text {Tits }}(R)$.

It is standard that there is a $W$-equivariant bijection $\alpha \mapsto \alpha^{\vee}$ from the roots $\Phi \subseteq \mathbb{Z}^{I}$ to their corresponding coroots in $\mathbb{Z}^{I \vee}$. As the notation suggests, the coroots corresponding to the simple roots $\alpha_{i}$ are our basis $\alpha_{i}^{\vee}$ for $\mathbb{Z}^{I \vee}$. In view of $W$-equivariance this determines the bijection uniquely. For $\alpha \in \Phi$ and $r \in R^{*}$ we define $h_{\alpha}(r) \in \operatorname{Aut} \mathfrak{S t}^{\mathrm{Tits}}(R)$ as $\operatorname{Ad}\left(r \otimes \alpha^{\vee}\right)$. As usual, we abbreviate $h_{\alpha_{i}}(r)$ to $h_{i}(r)$.

We define the Steinberg group functor $\mathfrak{S t}$ as follows. Informally, $\mathfrak{S t}(R)$ is the quotient of $\mathfrak{S t}^{\text {Tits }}(R)$ got by forcing every $\tilde{s}_{e}(r)$ to act on every $\mathfrak{U}_{\beta}(R)$ by $h_{\alpha}(r) \circ s_{e}^{*}$, where $\alpha$ is the root with $e \in E_{\alpha}$. Formally, it is the quotient by the subgroup normally generated by the elements

$$
\tilde{s}_{e}(r) u \tilde{s}_{e}(r)^{-1} \cdot\left(\left(h_{\alpha}(r) \circ s_{e}^{*}\right)(u)\right)^{-1}
$$

as $\alpha, \beta$ vary over $\Phi, e$ over $E_{\alpha}, r$ over $R^{*}$, and $u$ over $\mathfrak{U}_{\beta}(R)$. This set of relators is visibly $W^{*}$-invariant, so $W^{*}$ acts on $\mathfrak{S t}$.

Remark 6.2. Because $\tilde{s}_{e}(r)=\tilde{h}_{e}(r) \tilde{s}_{e}$, an equivalent way to impose the relations (6-5) is by quotienting by the subgroup of $\mathfrak{S t}^{\text {Tits }}(R)$ normally generated by all

$$
\begin{gathered}
\tilde{s}_{e} u \tilde{s}_{e}^{-1} \cdot s_{e}^{*}(u)^{-1} \\
\tilde{h}_{e}(r) u \tilde{h}_{e}(r)^{-1} \cdot\left(h_{\alpha}(r)(u)\right)^{-1}
\end{gathered}
$$

Remark 6.3. Our relations differ slightly from the relations $\left(\mathrm{B}^{\prime}\right)$ of [Morita and Rehmann 1990], because we follow Tits' convention for the presentation of $\mathfrak{g}$, while they follow Kac's convention (see Section 4). Our relations also differ from Tits' relations [1987, §3.6] in the definition of his Kac-Moody group functor, even taking into account that our $\tilde{h}_{i}(r)$ corresponds to his $r^{h_{i}}$. This is because Rémy observed [2002, §8.3.3] that Tits' relator (6), namely $\tilde{s}_{i}(r)^{-1} \cdot \tilde{s}_{i} \cdot r^{h_{i}}$, is in error. Rémy fixed it by replacing the first $r$ by $1 / r$. Our repair, by exchanging the last two terms, is equivalent. 
Theorem 6.4 (alternative defining relations for $\mathfrak{S t}$ ). The kernel of the natural map $\mathfrak{S t}^{\mathrm{Tits}}(R) \rightarrow \mathfrak{S t}(R)$ is the smallest normal subgroup containing the elements

$$
\begin{gathered}
\tilde{h}_{i}(r) \mathfrak{x}_{j}(t) \tilde{h}_{i}(r)^{-1} \cdot \mathfrak{x}_{j}\left(r^{A_{i j}} t\right)^{-1} \\
\tilde{h}_{i}(r) \tilde{s}_{j} \mathfrak{x}_{j}(t) \tilde{s}_{j}^{-1} \tilde{h}_{i}(r)^{-1} \cdot\left(\tilde{s}_{j} \mathfrak{x}_{j}\left(r^{-A_{i j}} t\right) \tilde{s}_{j}^{-1}\right)^{-1} \\
\tilde{s}_{i} u \tilde{s}_{i}^{-1} \cdot s_{i}^{*}(u)^{-1}
\end{gathered}
$$

for all $i, j \in I, r \in R^{*}, t \in R$ and $u \in \mathfrak{U}_{\beta}$, where $\beta$ may be any root. Furthermore, the identities

$$
\begin{aligned}
\tilde{s}_{i} \tilde{h}_{j}(r) \tilde{s}_{i}^{-1} & =\tilde{h}_{i}\left(r^{A_{j i}}\right)^{-1} \tilde{h}_{j}(r), \\
{\left[\tilde{h}_{i}(r), \tilde{h}_{j}\left(r^{\prime}\right)\right] } & =\tilde{h}_{j}\left(r^{A_{i j}} r^{\prime}\right) \tilde{h}_{j}\left(r^{A_{i j}}\right)^{-1} \tilde{h}_{j}\left(r^{\prime}\right)^{-1}
\end{aligned}
$$

hold in $\mathfrak{S t}(R)$, for all $i, j \in I, r, r^{\prime} \in R^{*}$.

Remark 6.5 (applicability to $\mathfrak{P S t}$ ). The proof below does not use the relations defining $\mathfrak{S t}^{\text {Tits }}$. So it shows that the subgroup of $*_{\alpha \in \Phi} \mathfrak{U}_{\alpha}(R)$ normally generated by the relators (6-5) is the same as the one normally generated by (6-8)-(6-10), and that (6-11)-(6-12) hold in the quotient. This is useful because we will use the same relations when defining the pre-Steinberg group $\mathfrak{P S} t$ in the next section.

Proof. We begin by showing that (6-8)-(6-10) are trivial in $\mathfrak{S t}(R)$. First, (6-10) is got from (6-5) by taking $e=e_{i}$ and $r=1$. Next, recall the definition of $\tilde{h}_{i}(r)$ as $\tilde{s}_{i}(r) \tilde{s}_{i}(-1)$ in (6-3), and that the defining relations (6-5) for $\mathfrak{S t}(R)$ say how $\tilde{s}_{i}(r)$ acts on every $\mathfrak{U}_{\beta}$. So $\tilde{h}_{i}(r)$ acts on every $\mathfrak{U}_{\beta}$ as

$h_{i}(r) \circ s_{i}^{*} \circ h_{i}(-1) \circ s_{i}^{*}=h_{i}(r) \circ h_{i}(-1) \circ\left(s_{i}^{*}\right)^{2}=h_{i}(r) \circ h_{i}(-1) \circ h_{i}(-1)=h_{i}(r)$.

Taking $\beta=\alpha_{j}$ gives (6-8). For (6-9), take $\beta=-\alpha_{j}$ and use the fact that $\tilde{s}_{j}$ swaps $\mathfrak{U}_{ \pm \alpha_{j}}$ (since it acts as $s_{j}^{*}$ ). This finishes the proof that (6-8)-(6-10) are trivial in $\mathfrak{S t}(R)$.

Now we write $N$ for the smallest normal subgroup of $\mathfrak{S t}^{\text {Tits }}(R)$ containing (6-8)-(6-10) and $\equiv$ for equality modulo $N$. We will show that (6-11)-(6-12) hold modulo $N$ and that the relators (6-6)-(6-7) are trivial modulo $N$. We will use relator (6-10) without explicit mention: modulo $N$, each $\tilde{s}_{i}$ acts on every $\mathfrak{U}_{\beta}$ as $s_{i}^{*}$.

First we establish (6-11)-(6-12). Starting from the definition of $\tilde{s}_{j}\left(r^{\prime}\right)$, we have

$$
\tilde{s}_{j}\left(r^{\prime}\right)=\mathfrak{x}_{j}\left(r^{\prime}\right) \mathfrak{x}_{-j}\left(1 / r^{\prime}\right) \mathfrak{x}_{j}\left(r^{\prime}\right) \equiv \mathfrak{x}_{j}\left(r^{\prime}\right) \cdot \tilde{s}_{j} \mathfrak{x}_{j}\left(1 / r^{\prime}\right) \tilde{s}_{j}^{-1} \cdot \mathfrak{x}_{j}\left(r^{\prime}\right) .
$$

Now the relators (6-8)-(6-9) give

$$
\tilde{h}_{i}(r) \tilde{s}_{j}\left(r^{\prime}\right) \tilde{h}_{i}(r)^{-1} \equiv \tilde{s}_{j}\left(r^{A_{i j}} r^{\prime}\right) .
$$


Taking $r^{\prime}=1$, left-multiplying by $\tilde{h}_{i}(r)^{-1}$, right-multiplying by $\tilde{s}_{j}^{-1}$, and then inverting both sides and using (6-4), gives

$$
\tilde{s}_{j} \tilde{h}_{i}(r) \tilde{s}_{j}^{-1} \equiv \tilde{s}_{j}(1) \tilde{s}_{j}\left(r^{A_{i j}}\right)^{-1} \tilde{h}_{i}(r) \equiv \tilde{h}_{j}\left(r^{A_{i j}}\right)^{-1} \tilde{h}_{i}(r) .
$$

Exchanging $i$ and $j$ establishes (6-11). Also, (6-13), (6-3) and (6-4) show that

$$
\tilde{h}_{i}(r) \tilde{h}_{j}\left(r^{\prime}\right) \tilde{h}_{i}(r)^{-1} \equiv \tilde{s}_{j}\left(r^{A_{i j}} r^{\prime}\right) \tilde{s}_{j}\left(r^{A_{i j}}\right)^{-1}=\tilde{h}_{j}\left(r^{A_{i j}} r^{\prime}\right) \tilde{h}_{j}\left(r^{A_{i j}}\right)^{-1} .
$$

Right-multiplication by $\tilde{h}_{j}\left(r^{\prime}\right)^{-1}$ gives (6-12).

Now we will prove (6-7) for all $e_{i}$. That is: modulo $N, \tilde{h}_{i}(r)$ acts on every $\mathfrak{U}_{\beta}$ by $h_{i}(r)$. To prove this, write $E$ for $\bigcup_{\beta \in \Phi} E_{\beta}$ and consider for any $e \in E$ the condition

$$
\tilde{h}_{i}(r) \mathfrak{x}_{e}(t) \tilde{h}_{i}(r)^{-1} \equiv \mathfrak{x}_{e}\left(r^{\left\langle\alpha_{i}^{\vee}, \beta\right\rangle} t\right) \quad \text { for all } i \in I, r \in R^{*} \text { and } t \in R,
$$

where $\beta$ is the root with $e \in E_{\beta}$. The set of $e \in E$ satisfying this condition is closed under negation, because $\mathfrak{x}_{-e}(t)=\mathfrak{x}_{e}(-t)$. This set contains $e_{j} \in E_{\alpha_{j}}$ and $f_{j} \in E_{-\alpha_{j}}$, for every $j \in I$, by relations (6-8)-(6-9). The next paragraph shows that it is closed under the action of $W^{*}$. Therefore, all $e \in E$ satisfy (6-15), establishing (6-7) for all $e=e_{i}$.

Here is the calculation that if $e \in E$ satisfies (6-15), and $j$ is any element of $I$, then $s_{j}^{*}(e)$ also satisfies (6-15). We must establish it for all $i$, so fix some $i \in I$. We have

$$
\begin{aligned}
& \tilde{h}_{i}(r) \mathfrak{x}_{s_{j}^{*}(e)}(t) \tilde{h}_{i}(r)^{-1} \\
& =\tilde{h}_{i}(r) \mathfrak{x}_{s_{j}^{*-1} \circ h_{j}(-1)(e)}(t) \tilde{h}_{i}(r)^{-1} \\
& \equiv \tilde{h}_{i}(r) \tilde{s}_{j}^{-1} \mathfrak{x}_{e}\left((-1)^{\left\langle\alpha_{j}^{\vee}, \beta\right\rangle} t\right) \tilde{s}_{j} \tilde{h}_{i}(r)^{-1} \\
& =\tilde{s}_{j}^{-1}\left(\tilde{s}_{j} \tilde{h}_{i}(r) \tilde{s}_{j}^{-1}\right) \mathfrak{x}_{e}\left((-1)^{\left\langle\alpha_{j}^{\vee}, \beta\right\rangle} t\right)\left(\tilde{s}_{j} \tilde{h}_{i}(r)^{-1} \tilde{s}_{j}^{-1}\right) \tilde{s}_{j} \\
& \equiv \tilde{s}_{j}^{-1}\left(\tilde{h}_{j}\left(r^{A_{i j}}\right)^{-1} \tilde{h}_{i}(r)\right) \mathfrak{x}_{e}\left((-1)^{\left\langle\alpha_{j}^{\vee}, \beta\right\rangle} t\right)\left(\tilde{h}_{i}(r)^{-1} \tilde{h}_{j}\left(r^{A_{i j}}\right)\right) \tilde{s}_{j} \\
& \equiv \tilde{s}_{j}^{-1} \mathfrak{x}_{e}\left((-1)^{\left\langle\alpha_{j}^{\vee}, \beta\right\rangle} r^{\left\langle\alpha_{i}^{\vee}, \beta\right\rangle} r^{-A_{i j}\left\langle\alpha_{j}^{\vee}, \beta\right\rangle} t\right) \tilde{s}_{j} \\
& \equiv \mathfrak{x}_{s_{j}^{*-1}(e)}\left((-1)^{\left\langle\alpha_{j}^{\vee}, \beta\right\rangle} r^{\left\langle\alpha_{i}^{\vee}, \beta\right\rangle} r^{-A_{i j}\left\langle\alpha_{j}^{\vee}, \beta\right\rangle} t\right) \\
& =\mathfrak{x}_{s_{j}^{*} \circ h_{j}(-1)(e)}\left((-1)^{\left\langle\alpha_{j}^{\vee}, \beta\right\rangle} r^{\left\langle\alpha_{i}^{\vee}, \beta\right\rangle} r^{-A_{i j}\left\langle\alpha_{j}^{\vee}, \beta\right\rangle} t\right) \\
& =\mathfrak{x}_{s_{j}^{*}(e)}\left(r^{\left\langle\alpha_{i}^{\vee}, \beta\right\rangle} r^{-A_{i j}\left\langle\alpha_{j}^{\vee}, \beta\right\rangle} t\right) . \\
& \text { by }\left(s_{j}^{*}\right)^{2}=h_{j}(-1)
\end{aligned}
$$

The right side of (6-15) for $s_{j}^{*}(e)$ has a similar form. Establishing equality amounts to showing $\left\langle\alpha_{i}^{\vee}-A_{i j} \alpha_{j}^{\vee}, \beta\right\rangle=\left\langle\alpha_{i}^{\vee}, s_{j}(\beta)\right\rangle$. This follows from $s_{j}(\beta)=\beta-$ $\left\langle\alpha_{j}^{\vee}, \beta\right\rangle \alpha_{j}$, finishing the proof of (6-15) for all $e \in E$. 
For $e$ equal to any $\pm e_{i}$, we were given (6-6) and we have proven (6-7). The same results for all $e$ follow by $W^{*}$ symmetry. More precisely, we claim that, for all $j \in I$, if (6-6) and (6-7) hold for some $e \in E$ then they hold for $s_{j}^{*}(e)$ too. We give the details for (6-7), and the argument is the same for (6-6). Suppose $r \in R^{*}$ and $u \in \bigcup_{\beta \in \Phi} \mathfrak{U}_{\beta}$. Then the left and right "sides" of the known relation (6-7) for $e$ lie in $\bigcup_{\beta \in \Phi} \mathfrak{U}_{\beta}$, so conjugating the left by $\tilde{s}_{j}$ has the same result as applying $s_{j}^{*}$ to the right. That is,

$$
\begin{aligned}
\tilde{s}_{j} \tilde{s}_{e} u \tilde{s}_{e}^{-1} \tilde{s}_{j}^{-1} & \equiv s_{j}^{*} \circ s_{e}^{*}(u), \\
\left(\tilde{s}_{j} \tilde{s}_{e} \tilde{s}_{j}^{-1}\right)\left(\tilde{s}_{j} u \tilde{s}_{j}^{-1}\right)\left(\tilde{s}_{j} \tilde{s}_{e}^{-1} \tilde{s}_{j}^{-1}\right) & \equiv s_{j}^{*} \circ s_{e}^{*} \circ s_{j}^{*-1} \circ s_{j}^{*}(u), \\
s_{s_{j}^{*}(e)}^{*} s_{j}^{*}(u)\left(s_{s_{j}^{*}(e)}^{*}\right)^{-1} & \equiv s_{s_{j}^{*}(e)}^{*}\left(s_{j}^{*}(u)\right) .
\end{aligned}
$$

As $u$ varies over all of $\bigcup_{\beta \in \Phi} \mathfrak{U}_{\beta}$, so does $s_{j}^{*}(u)$. This verifies relation (6-7) for $s_{j}^{*}(e)$.

\section{The pre-Steinberg group $\mathfrak{P S t}$}

In this section we define the pre-Steinberg group functor $\mathfrak{P S t}_{A}$ in the same way as $\mathfrak{S t}_{A}$, but omitting some of its Chevalley relations. So it has a natural map to $\mathfrak{S t}_{A}$. Then we will write down another group functor as a concrete presentation, and show in Theorem 7.12 that it equals $\mathfrak{P S t}_{A}$. Since $\mathfrak{P S t}_{A} \rightarrow \mathfrak{S t}_{A}$ is often an isomorphism (Theorem 1.1), this often gives a new presentation for $\mathfrak{S t}_{A}$. As discussed in the Introduction, it is simpler and more explicit than previous presentations, and special cases of it appear in Table 1.1 and Section 2. In the rest of this section we suppress the subscript $A$.

We call two roots $\alpha, \beta$ classically prenilpotent if $(\mathbb{Q} \alpha+\mathbb{Q} \beta) \cap \Phi$ is finite and $\alpha+\beta \neq 0$. Then they are prenilpotent, and lie in some $A_{1}, A_{1}^{2}, A_{2}, B_{2}$ or $G_{2} \operatorname{root}$ system. We define the pre-Steinberg group functor $\mathfrak{P S t}$ exactly as we did the Steinberg functor $\mathfrak{S t}$ (Section 6), except that when imposing the Chevalley relations we only vary $\alpha, \beta$ over the classically prenilpotent pairs rather than all prenilpotent pairs. We still impose the relations (6-5) of Morita-Rehmann, or equivalently (6-6)-(6-7) or (6-8)-(6-10). (See Remark 6.5 for why Theorem 6.4 applies with $\mathfrak{P S t}$ in place of $\mathfrak{S t}$.) Just as for $\mathfrak{S t}, W^{*}$ acts on $\mathfrak{P S t}$ because it permutes the defining relators.

There is an obvious natural map $\mathfrak{P S t} \rightarrow \mathfrak{S t}$, got by imposing the remaining Chevalley relations, coming from prenilpotent pairs that are not classically prenilpotent. If $\Phi$ is finite then every prenilpotent pair is classically prenilpotent, so $\mathfrak{P S t} \rightarrow \mathfrak{S} \mathfrak{t}$ is an isomorphism.

The rest of this section is devoted to writing down a presentation for $\mathfrak{P S t}$. We start by defining an analogue $\widehat{W}$ of the Weyl group. It is the quotient of the free 
group on formal symbols $S_{i \in I}$ by the subgroup normally generated by the words

$$
\begin{array}{ll}
\left(S_{i} S_{j} \cdots\right) \cdot\left(S_{j} S_{i} \cdots\right)^{-1} & \text { if } m_{i j} \neq \infty, \\
S_{i}^{2} S_{j} S_{i}^{-2} \cdot S_{j}^{-1} & \text { if } A_{i j} \text { is even, } \\
S_{i}^{2} S_{j} S_{i}^{-2} \cdot S_{j} & \text { if } A_{i j} \text { is odd, }
\end{array}
$$

where $i, j$ vary over $I$, and (7-1) has $m_{i j}$ terms inside each pair of parentheses, alternating between $S_{i}$ and $S_{j}$. These are called the Artin relators, for example, $S_{i} S_{j} S_{i} \cdot\left(S_{j} S_{i} S_{j}\right)^{-1}$ if $m_{i j}=3$.

Remark 7.1. We chose these defining relations so that $\widehat{W}$ would have four properties. First, it maps naturally to $W^{*}$, so that it acts on $\mathfrak{g}$ and $*_{\alpha \in \Phi} \mathfrak{U}_{\alpha}$. Second, the kernel of $\widehat{W} \rightarrow W$ is generated (not just normally) by the $S_{i}^{2}$. This plays a key role in the proof of Theorem 7.5 below. Third, each relation involves just two subscripts, which is needed for the Curtis-Tits property of $\mathfrak{P S t}$ (Corollary 1.3). And fourth, the $\tilde{s}_{i} \in \mathfrak{S t}$, defined in (7-27), satisfy the same relations. (Formally: $S_{i} \rightarrow \tilde{s}_{i}$ extends to a homomorphism $\widehat{W} \rightarrow \mathfrak{S t}$.) The first two properties are established in the next lemma, the third is obvious, and the fourth is part of Theorem 7.12.

Lemma 7.2 (basic properties of $\widehat{W}$ ). (i) $S_{i} \mapsto s_{i}^{*}$ defines a surjection $\widehat{W} \rightarrow W^{*}$.

(ii) $S_{j} S_{i}^{2} S_{j}^{-1}=S_{i}^{2}$ (resp. $\left.S_{j}^{2} S_{i}^{2}\right)$ if $A_{i j}$ is even (resp. odd).

(iii) The $S_{i}^{2}$ generate the kernel of the composition $\widehat{W} \rightarrow W^{*} \rightarrow W$.

Proof. We saw in Theorem 5.5 that the $s_{i}^{*}$ satisfy the Artin relations. Rewriting Lemma 5.2(ii)'s relation in $W^{*}$ with $i$ and $j$ reversed gives

$$
s_{j}^{*}\left(s_{i}^{*}\right)^{2} s_{j}^{*-1}=\left(s_{i}^{*}\right)^{2}\left(s_{j}^{*}\right)^{-2 A_{i j}} .
$$

Multiplying on the left by $s_{j}^{*-1}$ and on the right by $\left(s_{i}^{*}\right)^{-2}$, then inverting, gives

$$
\left(s_{i}^{*}\right)^{2} s_{j}^{*}\left(s_{i}^{*}\right)^{-2}=\left(s_{i}^{*}\right)^{2}\left(s_{j}^{*}\right)^{2 A_{i j}}\left(s_{i}^{*}\right)^{-2} s_{j}^{*}=\left(s_{j}^{*}\right)^{1+2 A_{i j}} .
$$

In the second step we used the fact that $s_{i}^{* 2}$ and $s_{j}^{* 2}$ commute. Using $s_{j}^{* 4}=1$, the right side is $s_{j}^{*}$ if $A_{i j}$ is even and $s_{j}^{*-1}$ if $A_{i j}$ is odd. This shows that $S_{i} \mapsto s_{i}^{*}$ sends the relators (7-2)-(7-3) to the trivial element of $W^{*}$, proving (i).

One can manipulate (7-2)-(7-3) in a similar way, yielding (ii). It follows immediately that the subgroup generated by the $S_{i}^{2}$ is normal. Because of the Artin relations, this is the kernel of $\widehat{W} \rightarrow W$. So we have proven (iii).

Remark 7.3. Though we don't need them, the following relations in $\widehat{W}$ show that $\widehat{W}$ is "not much larger" than $W^{*}$. First (7-2)-(7-3) imply the centrality of every $S_{i}^{4}$. Second, if some $A_{i j}$ is odd then (7-3) shows that $S_{j}^{ \pm 4}$ are conjugate; since both are central they must be equal, so $S_{j}^{8}=1$. Third, the relation obtained at the end of 
the proof implies $\left[S_{j}^{2}, S_{i}^{2}\right]=1$ or $S_{j}^{4}$, according to whether $A_{i j}$ is even or odd. In particular, these commutators are central. Finally, we can use this twice:

$$
\left\{\begin{array}{cc}
1 & \text { if } A_{i j} \text { is even } \\
S_{j}^{4} & \text { if } A_{i j} \text { is odd }
\end{array}\right\}=\left[S_{j}^{2}, S_{i}^{2}\right]=\left[S_{i}^{2}, S_{j}^{2}\right]^{-1}=\left\{\begin{array}{cc}
1 & \text { if } A_{j i} \text { is even } \\
S_{i}^{-4} & \text { if } A_{j i} \text { is odd }
\end{array}\right\} .
$$

In particular, if both $A_{i j}$ and $A_{j i}$ are odd then $S_{i}^{4}$ and $S_{j}^{4}$ are equal. If $A_{i j}$ is even while $A_{j i}$ is odd then we get $S_{i}^{4}=1$.

Now we begin our presentation in earnest. Ultimately, $\mathfrak{P S t}(R)$ will have generators $S_{i}$ and $X_{i}(t)$, with $i$ varying over $I$ and $t$ varying over $R$, and relators (7-1)-(7-26).

We first define a group functor $\mathfrak{G}_{1}$ by declaring that $\mathfrak{G}_{1}(R)$ is the quotient of the free group on the formal symbols $X_{i}(t)$, by the subgroup normally generated by the relators

$$
X_{i}(t) X_{i}(u) \cdot X_{i}(t+u)^{-1}
$$

for all $i \in I$ and $t, u \in R$. The following description of $\mathfrak{G}_{1}$ is obvious.

Lemma 7.4. $\mathfrak{G}_{1} \cong *_{i \in I} \mathfrak{U}_{i}$, via the correspondence $X_{i}(t) \leftrightarrow \mathfrak{x}_{i}(t)$.

Next we define a group functor $\mathfrak{G}_{2}$ as a certain quotient of the free product $\mathfrak{G}_{1} * \widehat{W}$. Namely, $\mathfrak{G}_{2}(R)$ is the quotient of $\mathfrak{G}_{1}(R) * \widehat{W}$ by the subgroup normally generated by the following relators, with $i$ and $j$ varying over $I$ and $t$ over $R$ :

$$
\begin{array}{cc}
S_{i}^{2} X_{j}(t) S_{i}^{-2} \cdot\left(X_{j}\left((-1)^{A_{i j}} t\right)\right)^{-1} & \\
{\left[S_{i}, X_{j}(t)\right]} & \text { if } m_{i j}=2, \\
S_{j} S_{i} X_{j}(t) \cdot\left(X_{i}(t) S_{j} S_{i}\right)^{-1} & \text { if } m_{i j}=3, \\
{\left[S_{i} S_{j} S_{i}, X_{j}(t)\right]} & \text { if } m_{i j}=4, \\
{\left[S_{i} S_{j} S_{i} S_{j} S_{i}, X_{j}(t)\right]} & \text { if } m_{i j}=6 .
\end{array}
$$

The next theorem is the key step in our development; see Section 8 for the proof. Although it is not at all obvious, we have presented $\left(*_{\alpha \in \Phi} \mathfrak{U}_{\alpha}\right) \rtimes \widehat{W}$. Therefore, we "have" the root groups $\mathfrak{U}_{\alpha}$ for all $\alpha$, not just simple $\alpha$. This sets us up for imposing the Chevalley relations in the next step.

Theorem 7.5. $\mathfrak{G}_{2}$ is the semidirect product of $*_{\alpha \in \Phi} \mathfrak{U}_{\alpha}$ by $\widehat{W}$, where $\widehat{W}$ acts on the free product via its homomorphism to $W^{*}$ and $W^{*}$ 's action on $*_{\alpha \in \Phi} \mathfrak{U}_{\alpha}$ is induced

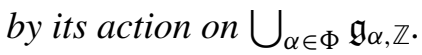

Remark 7.6 (groups with a root group datum). A Kac-Moody group over a field is an example of a group $G$ with a "root group datum". This means: a generating set of subgroups $\mathfrak{U}_{\alpha}$ parametrized by the roots $\alpha$ of a root system, permuted by (some extension $\widetilde{W}$ of) the Weyl group $W$ of that root system, and satisfying some additional hypotheses. See [Tits 1992] or [Caprace and Rémy 2009] for details. 
Examples include the Suzuki and Ree groups and isotropic forms of algebraic groups (or Kac-Moody groups) over fields. In many of these cases, some of the root groups are noncommutative. The heart of the proof of Theorem 7.5 is our understanding of root stabilizers in $W^{*}$ (Theorem 5.7), which would still apply in this more general setting. So there should be an analogous presentation of $\left(*_{\alpha \in \Phi} \mathfrak{U}_{\alpha}\right) \rtimes \widetilde{W}$. The main change would be to replace (7-4) by defining relations for $\mathfrak{U}_{i}$, and interpret the parameter $t$ of $X_{i}(t)$ as varying over some fixed copy of $\mathfrak{U}_{i}$, rather than over $R$. Since $G$ is a quotient of $\left(*_{\alpha \in \Phi} \mathfrak{U}_{\alpha}\right) \rtimes \widetilde{W}$, analogues of the rest of this section presumably yield a presentation of $G$.

Next we adjoin Chevalley relations corresponding to finite edges in the Dynkin diagram. That is, we define $\mathfrak{G}_{3}(R)$ as the quotient of $\mathfrak{G}_{2}(R)$ by the subgroup normally generated by the relators (7-10)-(7-23) below, for all $t, u \in R$. These are particular cases of the standard Chevalley relators, written in a form due to Demazure (see Remark 7.8 below).

When $i, j \in I$ with $m_{i j}=2$,

$$
\left[X_{i}(t), X_{j}(u)\right]
$$

When $i, j \in I$ with $m_{i j}=3$,

$$
\begin{aligned}
& {\left[X_{i}(t), S_{i} X_{j}(u) S_{i}^{-1}\right]} \\
& \quad\left[X_{i}(t), X_{j}(u)\right] \cdot S_{i} X_{j}(-t u) S_{i}^{-1}
\end{aligned}
$$

When $s, l \in I, m_{s l}=4$ and $s$ is the shorter root of the $B_{2}$,

$$
\begin{aligned}
& {\left[S_{s} X_{l}(t) S_{s}^{-1}, S_{l} X_{s}(u) S_{l}^{-1}\right]} \\
& {\left[X_{l}(t), S_{s} X_{l}(u) S_{s}^{-1}\right]} \\
& {\left[X_{s}(t), S_{l} X_{s}(u) S_{l}^{-1}\right] \cdot S_{s} X_{l}(2 t u) S_{s}^{-1}} \\
& \quad\left[X_{s}(t), X_{l}(u)\right] \cdot S_{s} X_{l}\left(-t^{2} u\right) S_{s}^{-1} \cdot S_{l} X_{s}(t u) S_{l}^{-1}
\end{aligned}
$$

When $s, l \in I, m_{s l}=6$ and $s$ is the shorter root of the $G_{2}$,

$$
\begin{gathered}
{\left[X_{l}(t), S_{l} S_{s} X_{l}(u) S_{s}^{-1} S_{l}^{-1}\right]} \\
{\left[S_{s} S_{l} X_{s}(t) S_{l}^{-1} S_{s}^{-1}, S_{l} S_{s} X_{l}(u) S_{s}^{-1} S_{l}^{-1}\right]} \\
{\left[S_{s} X_{l}(t) S_{s}^{-1}, S_{l} X_{s}(u) S_{l}^{-1}\right]} \\
{\left[X_{l}(t), S_{s} X_{l}(u) S_{s}^{-1}\right] \cdot S_{l} S_{s} X_{l}(-t u) S_{s}^{-1} S_{l}^{-1}} \\
{\left[X_{s}(t), S_{s} S_{l} X_{s}(u) S_{l}^{-1} S_{s}^{-1}\right] \cdot S_{s} X_{l}(-3 t u) S_{s}^{-1}}
\end{gathered}
$$




$$
\begin{aligned}
{\left[X_{s}(t), S_{l} X_{s}(u) S_{l}^{-1}\right] } & \cdot S_{l} S_{S} X_{l}\left(3 t u^{2}\right) S_{s}^{-1} S_{l}^{-1} \cdot S_{s} X_{l}\left(3 t^{2} u\right) S_{s}^{-1} \\
& \cdot S_{s} S_{l} X_{s}(2 t u) S_{l}^{-1} S_{s}^{-1} \\
{\left[X_{s}(t), X_{l}(u)\right] } & \cdot S_{l} S_{s} X_{l}\left(t^{3} u^{2}\right) S_{s}^{-1} S_{l}^{-1} \cdot S_{s} X_{l}\left(-t^{3} u\right) S_{s}^{-1} \\
& \cdot S_{l} X_{s}(t u) S_{l}^{-1} \cdot S_{s} S_{l} X_{s}\left(-t^{2} u\right) S_{l}^{-1} S_{s}^{-1}
\end{aligned}
$$

Remark 7.7 (asymmetry in the $A_{2}$ relators). The relators (7-11)-(7-12) are not symmetric in $i$ and $j$. Since $m_{j i}=3$ whenever $m_{i j}=3$, we are using both these relators and the ones got from them by exchanging $i$ and $j$.

Remark 7.8 (Demazure's form of the Chevalley relations). Our relators are written in a form due to Demazure (Propositions 3.2.1, 3.3.1 and 3.4.1 in [SGA $3_{\text {III }}$ 1970, Exposé XXIII]). They appear more complicated than the more usual one (for example, [Carter 1972, Theorem 5.2.2]), but have two important advantages. First, there are no implicit signs to worry about, and second, the presentation refers only to the Dynkin diagram, rather than the full root system.

One can convert (7-10)-(7-23) to a more standard form by working out which root groups contain the terms on the "right-hand sides" of the relators. For example, the term $S_{l} X_{s}(t u) S_{l}^{-1}$ of (7-23) lies in $S_{l} \mathfrak{U}_{s} S_{l}^{-1}=\mathfrak{U}_{\alpha_{s}+\alpha_{l}}$ because reflection in $\alpha_{l}$ sends $\alpha_{s}$ to $\alpha_{s}+\alpha_{l}$. Applying the same reasoning to the other terms, (7-23) equals $\left[X_{s}(t), X_{l}(u)\right]$ times a particular element of $\mathfrak{U}_{3 \alpha_{s}+2 \alpha_{l}} \cdot \mathfrak{U}_{2 \alpha_{s}+\alpha_{l}} \cdot \mathfrak{U}_{\alpha_{s}+\alpha_{l}} \cdot \mathfrak{U}_{2 \alpha_{s}+\alpha_{l}}$. The advantages of Demazure's form of the relators come from the fact that no identifications of these root groups with $R$ is required. We simply use the alreadyfixed identifications of the simple root groups with $R$, and transfer them to these other root groups by conjugation by $S_{s}$ and $S_{l}$.

Remark 7.9 (diagram automorphisms in characteristics 2 and 3). Some of the relators can be written in simpler but less symmetric ways. For example, (7-13) is the Chevalley relator for the roots $s_{s}\left(\alpha_{l}\right)$ and $s_{l}\left(\alpha_{s}\right)$ of $B_{2}$, which make angle $\pi / 4$. As we will see in the proof of Theorem 7.11, one could replace this pair of roots by any other pair of roots in the span of $\alpha_{s}, \alpha_{l}$ that make this angle. So, for example, one could replace (7-13) by the simpler relator $\left[S_{s} X_{l}(t) S_{s}^{-1}, X_{s}(u)\right]$. We prefer (7-13) because it maps to itself under the exceptional diagram automorphism in characteristic 2; see Section 3 for details. Similar considerations informed our choice of relators (7-18)-(7-19), and the ordering of the last four terms of (7-23).

Remark 7.10 (redundant relations). In practice, most of the relators coming from absent and single bonds in the Dynkin diagram, i.e., (7-10)-(7-12), can be omitted. Usually this reduces the size of the presentation greatly. See Propositions 9.1 and 9.2.

In Section 9 we prove the following more conceptual description of $\mathfrak{G}_{3}$. To be able to state it we use the temporary notation $\mathfrak{P S t}^{\text {Tits }}$ for the group functor defined 
in the same way as $\mathfrak{S t}^{\text {Tits }}$ (see Section 6), but only using classically prenilpotent pairs rather than all prenilpotent pairs. So $\mathfrak{P S t}^{\text {Tits }}$ is related to $\mathfrak{S t}^{\text {Tits }}$ in the same way that $\mathfrak{P S t}$ is related to $\mathfrak{S t}$. $\widehat{W}$ acts on $\mathfrak{P S t}^{\text {Tits }}$ for the same reason it acts on $\mathfrak{S t}^{\text {Tits }}$.

Theorem 7.11. The group functor $\mathfrak{P S t}^{\mathrm{Tits}} \rtimes \widehat{W}$ coincides with $\mathfrak{G}_{3}$. More precisely, under the identification $\mathfrak{G}_{2} \cong\left(*_{\alpha \in \Phi} \mathfrak{U}_{\alpha}\right) \rtimes \widehat{W}$ of Theorem 7.5 , the kernels of $\mathfrak{G}_{2} \rightarrow \mathfrak{G}_{3}$ and $\left(*_{\alpha \in \Phi} \mathfrak{U}_{\alpha}\right) \rtimes \widehat{W} \rightarrow \mathfrak{P S t}^{\text {Tits }} \rtimes \widehat{W}$ coincide.

Finally, we define $\mathfrak{G}_{4}$ as the quotient of $\mathfrak{G}_{3}$ by the smallest normal subgroup containing the relators

$$
\begin{gathered}
\tilde{h}_{i}(r) X_{j}(t) \tilde{h}_{i}(r)^{-1} \cdot X_{j}\left(r^{A_{i j}} t\right)^{-1} \\
\tilde{h}_{i}(r) S_{j} X_{j}(t) S_{j}^{-1} \tilde{h}_{i}(r)^{-1} \cdot S_{j} X_{j}\left(r^{-A_{i j}} t\right)^{-1} S_{j}^{-1} \\
S_{i} \cdot \tilde{s}_{i}(1)^{-1}
\end{gathered}
$$

where $r$ varies over $R^{*}, t$ over $R$ and $i, j$ over $I$. We are using the definitions

$$
\begin{aligned}
\tilde{s}_{i}(r) & :=X_{i}(r) S_{i} X_{i}(1 / r) S_{i}^{-1} X_{i}(r), \\
\tilde{h}_{i}(r) & :=\tilde{s}_{i}(r) \tilde{s}_{i}(-1) .
\end{aligned}
$$

Note that this definition of $\tilde{s}_{i}(r)$ is compatible with the one in Section 6, because $X_{i}(r) \in \mathfrak{G}_{3}$ corresponds to $\mathfrak{x}_{e_{i}}(r) \in \mathfrak{P S t}^{\text {Tits }}$ under the isomorphism of Lemma 7.4, while $S_{i} X_{i}(1 / r) S_{i}^{-1}$ corresponds to $s_{i}^{*}\left(\mathfrak{x}_{e_{i}}(1 / r)\right)=\mathfrak{x}_{f_{i}}(1 / r)$. As before, we will abbreviate $\tilde{s}_{i}(1)$ to $\tilde{s}_{i}$.

The following theorem is the main result of this section and a restatement of Theorem 1.2 from the Introduction.

Theorem 7.12 (presentation of the pre-Steinberg group $\mathfrak{P S t}$ ). The group functor $\mathfrak{P S t}$ coincides with $\mathfrak{G}_{4}$. In particular, for any commutative ring $R, \mathfrak{P S t}(R)$ has a presentation with generators $S_{i}$ and $X_{i}(t)$ for $i \in I$ and $t \in R$, and relators (7-1)-(7-26).

Proof. By definition, $\mathfrak{G}_{4}$ is the quotient of $\mathfrak{G}_{3}$ by the relations (7-24)-(7-26). Because $S_{i}$ acts on each $\mathfrak{U}_{\beta}$ by $s_{i}^{*}$ (Theorem 7.5), imposing (7-26) forces $\tilde{s}_{i}$ to also act this way. We consider the intermediate group $\mathfrak{G}_{3.5}$, of fleeting interest, got from $\mathfrak{G}_{3}$ by imposing (7-24)-(7-25) and the relations that $\tilde{s}_{i}$ acts on every $\mathfrak{U}_{\beta}$ as $s_{i}^{*}$ does. In other words, we are imposing on $\mathfrak{P S t}^{\text {Tits }} \subseteq \mathfrak{P S t}^{\text {Tits }} \rtimes \widehat{W}=\mathfrak{G}_{3}$ the relations (6-8)-(6-10). Theorem 6.4 and Remark 6.5 show that this reduces $\mathfrak{G}_{3}$ to $\mathfrak{P S t} \rtimes \widehat{W}$.

So $\mathfrak{G}_{4}$ is the quotient of $\mathfrak{G}_{3.5}=\mathfrak{P S} \mathfrak{t} \rtimes \widehat{W}$ by the relations $S_{i}=\tilde{s}_{i}$. We use Tietze transformations to eliminate the $S_{i}$ from the presentation, in favor of the $\tilde{s}_{i}$. So $\mathfrak{G}_{4}$ is the quotient of $\mathfrak{P S t}$ by the subgroup normally generated by the words got 
by replacing $S_{i}$ by $\tilde{s}_{i}$ in each of the relators (7-1)-(7-25). All of these relators are already trivial in $\mathfrak{P S t}$, so $\mathfrak{G}_{4}=\mathfrak{P S t}$.

In more detail, (7-1) requires the $\tilde{s}_{i}$ to satisfy the Artin relations, which they do in $\mathfrak{P S t}$ by [Tits 1987, (d) on p. 551]. The remaining relations (7-2)-(7-25) involve the $S_{i}$ only by their conjugacy action. For example, (7-17) says that $X_{l}(t)$ commutes with the conjugate of $X_{l}(u)$ by a certain word in $S_{s}$ and $S_{l}$. Since $S_{i}$ acts as $s_{i}^{*}$ by Theorem 7.5 and $\tilde{s}_{i}$ acts the same way by the definition of $\mathfrak{P S t}$, these relations still hold after replacing each $S_{i}$ by the corresponding $\tilde{s}_{i}$. (When defining $\widehat{W}$, we were careful not to impose any relations on the $S_{i}$ except those which are also satisfied by the $\tilde{s}_{i}$.)

Remark 7.13 (redundant relators). In most cases of interest, $A$ is 2-spherical without $A_{1}$ components. Then one can forget the relators (7-24)-(7-25) because they follow from previous relations. More specifically, suppose $m_{i j}$ is 3,4 or 6 . Then the relators (7-24)-(7-25) are already trivial in $\mathfrak{G}_{3}$. The same holds if $i=j$ and there exists some $k \in I$ with $m_{i k} \in\{3,4,6\}$. See [Tits 1987, $\left(\mathrm{a}_{4}\right)$, p. 550] for details.

Remark 7.14 (more redundant relators). One need only impose the relators (7-26) for a single $i$ in each component $\Omega$ of the "odd Dynkin diagram" $\Delta^{\text {odd }}$ considered in Section 5. This is because if $m_{i j}=3$ then $S_{i} S_{j}$ conjugates $S_{i}$ to $S_{j}$ and $X_{i}(t)$ to $X_{j}(t)$. This uses relators (7-1) and (7-7).

Remark 7.15 (precautions against typographical errors). We found explicit matrices for our generators, in standard representations of the $A_{1}^{2}, A_{2}, B_{2}$ and $G_{2}$ Chevalley groups over $\mathbb{Z}\left[r^{ \pm 1}, t, u\right]$. Then we checked on the computer that they satisfy the defining relations (7-1)-(7-26).

\section{The isomorphism $\mathfrak{G}_{2} \cong\left(*_{\alpha \in \Phi} \mathfrak{U}_{\alpha}\right) \rtimes \widehat{W}$}

In this section we will suppress the dependence of group functors on the base ring $R$, always meaning the group of points over $R$. Our goal is to prove Theorem 7.5, namely that the group $\mathfrak{G}_{2}$ with generators $S_{i}$ and $X_{i}(t), i \in I$ and $t \in R$, modulo the subgroup normally generated by the relators (7-1)-(7-9), is $\left(*_{\alpha \in \Phi} \mathfrak{U}_{\alpha}\right) \rtimes \widehat{W}$. The genesis of the theorem is the following elementary principle. It seems unlikely to be new, but I have not seen it before.

Lemma 8.1. Suppose $G=\left(*_{\alpha \in \Phi} U_{\alpha}\right) \rtimes H$, where $\Phi$ is some index set, the $U_{\alpha}$ are groups isomorphic to each other, and $H$ is a group whose action on the free product permutes the displayed factors transitively. Then $G \cong\left(U_{\infty} \rtimes H_{\infty}\right) *_{\infty} H$, where $\infty$ is some element of $\Phi$ and $H_{\infty}$ is its $H$-stabilizer.

Proof. The idea is that $U_{\infty} \rtimes H_{\infty} \mapsto\left(U_{\infty} \rtimes H_{\infty}\right) *_{\infty} H$ is a sort of free-product analogue of inducing a representation from $H_{\infty}$ to $H$. We suppress the subscript $\infty$ 
from $U_{\infty}$. Take a set $Z$ of left coset representatives for $H_{\infty}$ in $H$, and for $u \in U$ and $z \in Z$ define $u_{z}:=z u z^{-1} \in G$. The $u_{z}$ for fixed $z$ form the free factor $z U z^{-1}=U_{z(\infty)}$ of $\left(*_{\alpha \in \Phi} U_{\alpha}\right) \subseteq G$. Assuming $U \neq 1$, every displayed free factor occurs exactly once this way, since $H$ 's action on $\Phi$ is the same as on $H_{\infty}$ 's left cosets. So the maps $u_{z} \mapsto z u z^{-1} \in\left(U \rtimes H_{\infty}\right) *_{H_{\infty}} H$ define a homomorphism $\left(*_{\alpha \in \Phi} U_{\alpha}\right) \rightarrow$ $\left(U \rtimes H_{\infty}\right) *_{H_{\infty}} H$. This homomorphism is obviously $H$-equivariant, so it extends to a homomorphism $G \rightarrow\left(U \rtimes H_{\infty}\right) *_{H_{\infty}} H$. It is easy to see that this is inverse to the obvious homomorphism $\left(U \rtimes H_{\infty}\right) *_{\infty} H \rightarrow G$.

Now we begin proving Theorem 7.5 by reducing it to Lemma 8.2 below, which is an analogue of Theorem 7.5 for a single component of the "odd Dynkin diagram" $\Delta^{\text {odd }}$ introduced in Section 5. It is well-known that two generators $s_{i}, s_{j}$ of $W$ $(i, j \in I)$ are conjugate in $W$ if and only if $i$ and $j$ lie in the same component of $\Delta^{\text {odd }}$. (If $m_{i j}=3$ then $s_{i} s_{j} s_{i}=s_{j} s_{i} s_{j}$ implies the conjugacy of $s_{i}$ and $s_{j}$, while distinct components of $\Delta^{\text {odd }}$ correspond to different elements of the abelianization of $W$.)

Let $\Omega$ be one of these components, and write $\Phi(\Omega) \subseteq \Phi$ for the roots whose reflections are conjugate to some (hence any) $s_{i \in \Omega}$. Because $\Phi(\Omega)$ is a $W$-invariant subset of $\Phi$, we may form the group $\left(*_{\alpha \in \Phi(\Omega)} \mathfrak{U}_{\alpha}\right) \rtimes \widehat{W}$ just as we $\operatorname{did}\left(*_{\alpha \in \Phi} \mathfrak{U}_{\alpha}\right) \rtimes \widehat{W}$. We will write $\mathfrak{G}_{2, \Omega}$ for the group having generators $S_{i}$, with $i \in I$, and $X_{i}(t)$, with $i \in \Omega$ and $t \in R$, modulo the subgroup normally generated by the relators (7-1)-(7-3), and those relators (7-4)-(7-9) with $i \in \Omega$. Note that (7-7) is relevant only if $m_{i j}=3$, in which case $i \in \Omega$ if and only if $j \in \Omega$, so the relator makes sense. Caution: the subscripts on $S$ vary over all of $I$, while those on $X$ vary only over $\Omega \subseteq I$.

Lemma 8.2. For any component $\Omega$ of $\Delta^{\text {odd }}$,

$$
\mathfrak{G}_{2, \Omega} \cong\left(*_{\alpha \in \Phi(\Omega)} \mathfrak{U}_{\alpha}\right) \rtimes \widehat{W} .
$$

Proof of Theorem 7.5, given Lemma 8.2. An examination of the presentation of $\mathfrak{G}_{2}$ reveals that the $X$ 's corresponding to different components of $\Delta^{\text {odd }}$ don't interact. Precisely: $\mathfrak{G}_{2}$ is the amalgamated free product of the $\mathfrak{G}_{2, \Omega}$, where $\Omega$ varies over the components of $\Delta^{\text {odd }}$ and the amalgamation is that the copies of $\widehat{W}$ in the $\mathfrak{G}_{2, \Omega}$ are identified in the obvious way. Lemma 8.2 shows that $\mathfrak{G}_{2, \Omega}=\left(*_{\alpha \in \Phi(\Omega)} \mathfrak{U}_{\alpha}\right) \rtimes \widehat{W}$ for each $\Omega$. Taking their free product, amalgamated along their copies of $\widehat{W}$, obviously yields $\left(*_{\alpha \in \Phi} \mathfrak{U}_{\alpha}\right) \rtimes \widehat{W}$.

The rest of the section is devoted to proving Lemma 8.2. So we fix a component $\Omega$ of $\Delta^{\text {odd }}$ and phrase our problem in terms of the free product $F:=\left(*_{j \in \Omega} \mathfrak{U}_{j}\right) * \widehat{W}$. This is the group with generators $S_{i \in I}$ and $X_{j \in \Omega}(t)$, whose relations are (7-1)-(7-3) and those cases of (7-4) with $i \in \Omega$. The heart of the proof of Lemma 8.2 is to define normal subgroups $M, N$ of $F$ and show they are equal. $M$ turns out to be normally generated by the relators from (7-5)-(7-9) for which $i \in \Omega$. Given this, 
$\mathfrak{G}_{2, \Omega}=F / M$ by definition. The other group $F / N$ has a presentation like the one in Lemma 8.1. But it requires some preparation even to define, so we begin with an informal overview.

Start with the presentation of $\mathfrak{G}_{2, \Omega}$, and distinguish some point $\infty$ of $\Omega$ and a spanning tree $T$ for $\Omega$. We will use the relators (7-7) coming from the edges of $T$ to rewrite the $X_{j \in \Omega-\{\infty\}}(t)$ in terms of $X_{\infty}(t)$, and then eliminate the $X_{j \in \Omega-\{\infty\}}(t)$ from the presentation. This "uses up" those relators and makes the other relators messier because each $X_{j \neq \infty}(t)$ must be replaced by a word in $X_{\infty}(t)$ and elements of $\widehat{W}$. We studied the $W^{*}$-stabilizer of $\alpha_{\infty}$ in Theorem 5.7, and how it acts on $\mathfrak{g}_{\infty}$, hence on $\mathfrak{U}_{\infty}$. It turns out that the remaining relations in $\mathfrak{G}_{2, \Omega}$ are exactly the relations that the $\widehat{W}$-stabilizer $\widehat{W}_{\infty}$ of $\alpha_{\infty}$ acts on $\mathfrak{U}_{\infty}$ via $\widehat{W}_{\infty} \rightarrow \widehat{W} \rightarrow W^{*} \subseteq$ Aut $\mathfrak{g}$. That is, $\mathfrak{G}_{2, \Omega} \cong\left(\mathfrak{U}_{\infty} \rtimes \widehat{W}_{\infty}\right) * \widehat{W}_{\infty} \widehat{W}$. Then Lemma 8.1 identifies this with $\left(*_{\alpha \in \Phi(\Omega)} \mathfrak{U}_{\alpha}\right) \rtimes \widehat{W}$.

Now we proceed to the formal proof, beginning by defining some elements of $F$. For $\gamma$ an edge path in $\Omega$, with $i_{0}, \ldots, i_{n}$ the vertices along it, define $\alpha(\gamma)=i_{0}$ and $\omega(\gamma)=i_{n}$ as its initial and final endpoints, and define $P_{\gamma}$ by (5-3) with $S$ 's in place of $s$ 's. For $k \in I$ evenly joined to the end of $\gamma$ (i.e., $m_{k \omega(\gamma)}$ finite and even), define

$$
R_{\gamma, k}=P_{\gamma}^{-1} \cdot\left\{\begin{array}{c}
S_{k} \\
S_{k} S_{\omega(\gamma)} S_{k} \\
S_{k} S_{\omega(\gamma)} S_{k} S_{\omega(\gamma)} S_{k}
\end{array}\right\} \cdot P_{\gamma}
$$

according to whether $m_{k \omega(\gamma)}=2,4$ or 6 . (We get $R_{\gamma, k}$ from (5-4) by replacing $s$ 's and $p$ 's by $S$ 's and $P$ 's, and $j$ by $\omega(\gamma)$.) Next, for $t \in R$ we define

$$
C_{\gamma}(t):=P_{\gamma} X_{\alpha(\gamma)}(t) \cdot\left(X_{\omega(\gamma)}(t) P_{\gamma}\right)^{-1},
$$

and for $k \in I$ evenly joined to $\omega(\gamma)$ we define

$$
D_{\gamma, k}(t):=\left[R_{\gamma, k}, X_{\alpha(\gamma)}(t)\right] .
$$

For ease of reference we will also give the name

$$
B_{i j}(t):=S_{i}^{2} X_{j}(t) S_{i}^{-2} \cdot X_{j}\left((-1)^{A_{i j}} t\right)^{-1}
$$

to the word (7-5), where $i \in I$ and $j \in \Omega$. We will suppress the dependence of the $X_{j}, B_{i j}, C_{\gamma}$ and $D_{\gamma, k}$ on $t$ except where it plays a role.

The following formally meaningless intuition may help the reader; Lemma 8.3 below gives it some support. The relation $C_{\gamma}=1$ declares that the path $\gamma$ conjugates the $X$ "at" the beginning of $\gamma$ to the $X$ "at" the end. And the relation $D_{\gamma, k}=1$ declares that the $X$ "at" the beginning of $\gamma$ commutes with a certain word that corresponds to going along $\gamma$, going around some sort of "loop based at the endpoint of $\gamma$ ", and then retracing $\gamma$.

Our first normal subgroup $M$ of $F$ is defined as the subgroup normally generated by all the $B_{i j}$, the $C_{\gamma}$ for all $\gamma$ of length 1 , and the $D_{\gamma, k}$ for all $\gamma$ of length 0 . 
Unwinding the definitions shows that these elements of $F$ are exactly the ones we used in defining $\mathfrak{G}_{2, \Omega}$. For example, if $\gamma$ is the length- 1 path from one vertex $j$ of $\Omega$ to an adjacent vertex $i$ then $P_{\gamma}=S_{j} S_{i}$ and $C_{\gamma}$ is the word (7-7). And if $i \in \Omega$ is evenly joined to $j \in I$ then we take $\gamma$ to be the zero-length path at $i$, and $D_{\gamma, j}$ turns out to be the relator (7-6), (7-8) or (7-9). Which one of these applies depends on $m_{i j} \in\{2,4,6\}$. So $F / M \cong \mathfrak{G}_{2, \Omega}$.

Before defining the other normal subgroup $N$, we explain how to work with the $C$ 's and $D$ 's by thinking in terms of paths rather than complicated words.

Lemma 8.3. Suppose $\gamma_{1}$ and $\gamma_{2}$ are paths in $\Omega$ with $\omega\left(\gamma_{1}\right)=\alpha\left(\gamma_{2}\right)$, and let $\gamma$ be the path which traverses $\gamma_{1}$ and then $\gamma_{2}$ :

(i) Any normal subgroup of $F$ containing two of $C_{\gamma_{1}}, C_{\gamma_{2}}$ and $C_{\gamma}$ contains the third.

(ii) Suppose $k \in I$ is evenly joined to $\omega\left(\gamma_{2}\right)$. Then any normal subgroup of $F$ containing $C_{\gamma_{1}}$ and one of $D_{\gamma_{2}, k}$ and $D_{\gamma, k}$ contains the other as well.

Proof. Both identities

$$
\begin{aligned}
C_{\gamma} & =\left(P_{\gamma_{2}} C_{\gamma_{1}} P_{\gamma_{2}}^{-1}\right) C_{\gamma_{2}}, \\
D_{\gamma, k} & =P_{\gamma_{1}}^{-1}\left(\left(R_{\gamma_{2}, k} C_{\gamma_{1}} R_{\gamma_{2}, k}^{-1}\right) D_{\gamma_{2}, k} C_{\gamma_{1}}^{-1}\right) P_{\gamma_{1}}
\end{aligned}
$$

unravel to tautologies, using $P_{\gamma}=P_{\gamma_{2}} P_{\gamma_{1}}$. These imply (i) and (ii), respectively.

To define $N$ we refer to the base vertex $\infty$ and spanning tree $T$ that we introduced above. For each $j \in \Omega$ we take $\delta_{j}$ to be the backtracking-free path in $T$ from $\infty$ to $j$. For each edge of $\Omega$ not in $T$, choose an orientation of it, and define $\mathcal{E}$ as the corresponding set of paths of length 1 . For $\gamma \in \mathcal{E}$ we write $z(\gamma)$ for the corresponding loop in $\Omega$ based at $\infty$. That is, $z(\gamma)$ is $\delta_{\alpha(\gamma)}$ followed by $\gamma$ followed by the reverse of $\delta_{\omega(\gamma)}$. We define $Z$ as $\{z(\gamma) \mid \gamma \in \mathcal{E}\}$, which is a free basis for the fundamental group $\pi_{1}(\Omega, \infty)$. We define $N$ as the subgroup of $F$ normally generated by all $B_{i \infty}$ with $i \in I$, all $C_{z \in Z}$, the $C_{\delta_{j}}$ with $j \in \Omega$, and all $D_{\delta_{j}, k}$ where $j \in \Omega$ and $k \in I$ are evenly joined. We will show $M=N$; one direction is easy:

\section{Lemma 8.4. $M$ contains $N$.}

Proof. Since $M$ contains $C_{\gamma}$ for every length-1 path $\gamma$, repeated applications of Lemma 8.3(i) show that it contains the $C_{\delta_{i}}$ and $C_{z \in Z}$. Since $M$ contains $D_{\gamma, k}$ for every $\gamma$ of length 0 , part (ii) of the same lemma shows that $M$ also contains the $D_{\delta_{j}, k}$. Since $M$ contains all the $B_{i j}$, not just the $B_{i \infty}$, the proof is complete.

Now we set about proving the reverse inclusion. For convenience we use $\equiv$ to mean "equal modulo $N$ ". We must show that each generator of $M$ is $\equiv 1$.

Lemma 8.5. $C_{\gamma} \equiv 1$ for every length-1 subpath $\gamma$ of every $\delta_{j}$.

Proof. This follows from Lemma 8.3(i) because $\delta_{\alpha(\gamma)}$ followed by $\gamma$ is $\delta_{\omega(\gamma)}$. 
Lemma 8.6. $B_{i k} \equiv 1$ for all $i \in I$ and $k \in \Omega$.

Proof. We claim that if $\gamma$ is a length-1 path in $\Omega$, such that $C_{\gamma} \equiv 1$ and $B_{i \alpha(\gamma)} \equiv 1$ for every $i \in I$, then also $B_{i \omega(\gamma)} \equiv 1$ for every $i \in I$. Assuming this, we use the fact that $B_{i \infty} \equiv 1$ for all $i \in I$ and also $C_{\gamma} \equiv 1$ for every length-1 subpath $\gamma$ of every $\delta_{k}$ (Lemma 8.5). Since every $k \in \Omega$ is the end of chain of such $\gamma$ 's starting at $\infty$, the lemma follows by induction.

So now we prove the claim, writing $i$ for some element of $I$ and $j$ and $k$ for the initial and final endpoints of $\gamma$. We use $C_{\gamma} \equiv 1$, i.e., $S_{j} S_{k} X_{j}(t) \equiv X_{k}(t) S_{j} S_{k}$, to get

$$
\begin{aligned}
S_{i}^{2} X_{k}(t) S_{i}^{-2} & \equiv S_{i}^{2} S_{j} S_{k} X_{j}(t) S_{k}^{-1} S_{j}^{-1} S_{i}^{-2} \\
& =S_{j} S_{k}\left[\left(S_{k}^{-1} S_{j}^{-1} S_{i}^{2} S_{j} S_{k}\right) X_{j}(t)\left(S_{k}^{-1} S_{j}^{-1} S_{i}^{-2} S_{j} S_{k}\right)\right] S_{k}^{-1} S_{j}^{-1} .
\end{aligned}
$$

We rewrite the relation from Lemma 7.2(ii) as $S_{j}^{-1} S_{i}^{2} S_{j}=S_{j}^{(-1)^{A_{i j}}-1} S_{i}^{2}$. Then we use it and its analogues with subscripts permuted to simplify the first parenthesized term in (8-1). We also use $A_{j k}=-1$, which holds since $j$ and $k$ are joined. The result is

$$
S_{k}^{-1} S_{j}^{-1} S_{i}^{2} S_{j} S_{k}=S_{k}^{1-(-1)^{A_{i j}}} S_{j}^{(-1)^{A_{i j}}-1} S_{k}^{-1+(-1)^{A_{i k}}} S_{i}^{2} .
$$

Note that each exponent is 0 or \pm 2 .

The bracketed term in (8-1) is the conjugate of $X_{j}(t)$ by this. We work this out in four steps, using our assumed relations $B_{i j} \equiv B_{j j} \equiv B_{k j} \equiv 1$. Conjugation by $S_{i}^{2}$ changes $X_{j}(t)$ to $X_{j}\left((-1)^{A_{i j}} t\right)$. Because $A_{k j}=-1$, conjugating $X_{j}\left((-1)^{A_{i j}} t\right)$ by $S_{k}^{(-1)^{A_{i k}-1}}$ sends it to

$$
\begin{cases}\text { itself } & \text { if } A_{i k} \text { is even, because }(-1)^{A_{i k}}-1=0 \\ X_{j}\left(-(-1)^{A_{i j}} t\right) & \text { if } A_{i k} \text { is odd, because }(-1)^{A_{i k}}-1=-2\end{cases}
$$

We write this as $X_{j}\left((-1)^{A_{i k}}(-1)^{A_{i j}} t\right)$. In the third step we conjugate by an even power of $S_{j}$, which does nothing. The fourth step is like the second, and introduces a second factor $(-1)^{A_{i j}}$. The net result is that the bracketed term of (8-1) equals $X_{j}\left((-1)^{A_{i k}} t\right)$ modulo $N$.

Plugging this into (8-1) and then using the conjugacy relation $C_{\gamma} \equiv 1$ between $X_{j}$ and $X_{k}$ yields

$$
S_{i}^{2} X_{k}(t) S_{i}^{-2} \equiv S_{j} S_{k} X_{j}\left((-1)^{A_{i k}} t\right) S_{k}^{-1} S_{j}^{-1} \equiv X_{k}\left((-1)^{A_{i k}} t\right) .
$$

We have established the desired relation $B_{i k} \equiv 1$.

Lemma 8.7. Suppose $\gamma$ is a length-1 path in $\Omega$ with $C_{\gamma} \equiv 1$. Then $C_{\text {reverse }(\gamma)} \equiv 1$ also.

Proof. Suppose $\gamma$ goes from $j$ to $k$. We begin with our assumed relation $C_{\gamma} \equiv 1$, i.e., $S_{j} S_{k} X_{j}(t) \equiv X_{k}(t) S_{j} S_{k}$, rearrange and apply the relation from Lemma 7.2(ii) 
with $A_{j k}=$ odd:

$$
\begin{aligned}
X_{k}(t) & \equiv S_{j} S_{k} X_{j}(t) S_{k}^{-1} S_{j}^{-1} \\
S_{k} S_{j} X_{k}(t) & \equiv\left(S_{k} S_{j}^{2} S_{k}^{-1}\right) S_{k}^{2} X_{j}(t) S_{k}^{-1} S_{j}^{-1}=\left(S_{k}^{2} S_{j}^{2}\right) S_{k}^{2} X_{j}(t) S_{k}^{-1} S_{j}^{-1} .
\end{aligned}
$$

Now we simplify the right side using Lemma 8.6's $B_{j j} \equiv B_{k j} \equiv 1$ with $A_{k j}=$ odd:

$$
\begin{aligned}
\left(S_{k}^{2} S_{j}^{2}\right) S_{k}^{2} X_{j}(t) S_{k}^{-1} S_{j}^{-1} & \equiv S_{k}^{2} S_{j}^{2} X_{j}(-t) S_{k}^{2} S_{k}^{-1} S_{j}^{-1} \\
& \equiv S_{k}^{2} X_{j}(-t) S_{j}^{2} \cdot S_{k}^{2} S_{k}^{-1} S_{j}^{-1} \\
& \equiv X_{j}(t) S_{k}^{2} S_{j}^{2} \cdot S_{k}^{2} S_{k}^{-1} S_{j}^{-1} \\
& =X_{j}(t) S_{k} S_{j}^{2} S_{k}^{-1} \cdot S_{k}^{2} S_{k}^{-1} S_{j}^{-1} \\
& =X_{j}(t) S_{k} S_{j} .
\end{aligned}
$$

We have shown $C_{\text {reverse }(\gamma)} \equiv 1$, as desired.

Lemma 8.8. $M=N$. In particular, $\mathfrak{G}_{2, \Omega}$ is the quotient of $F=\left(*_{j \in \Omega} \mathfrak{U}_{j}\right) * \widehat{W}$ by $N$.

Proof. We showed $N \subseteq M$ in Lemma 8.4. To show the reverse inclusion, recall that $M$ is normally generated by all $B_{i j}$, the $C_{\gamma}$ for all $\gamma$ of length 1 , and the $D_{\gamma, k}$ for all $\gamma$ of length 0 . We must show that each of these is $\equiv 1$. We showed $B_{i j} \equiv 1$ in Lemma 8.6.

Next we show that $C_{\gamma} \equiv 1$ for every length-1 path $\gamma$ in $T$. If $\gamma$ is part of one of the paths $\delta_{j}$ in $T$ based at $\infty$, then $C_{\gamma} \equiv 1$ by Lemma 8.5 , and then $C_{\text {reverse }(\gamma)} \equiv 1$ by Lemma 8.7 .

Lemma 8.3(i) now shows $C_{\gamma} \equiv 1$ for every path $\gamma$ in $T$.

Next we show $C_{\gamma} \equiv 1$ for every length-1 path $\gamma$ not in $T$. Recall that we chose a set $\mathcal{E}$ of length-1 paths, one traversing each edge of $\Omega$ not in $T$. For $\gamma \in \mathcal{E}$ we wrote $z(\gamma)$ for the corresponding loop in $\Omega$ based at $\infty$, namely $\delta_{\alpha(\gamma)}$ followed by $\gamma$ followed by reverse $\left(\delta_{\omega(\gamma)}\right)$. Recall that $N$ contains $C_{z(\gamma)}$ by definition, and contains $C_{\delta_{\alpha(\gamma)}}$ and $C_{\text {reverse }\left(\delta_{\omega(\gamma)}\right)}$ by the previous paragraph. So a double application of Lemma 8.3(i) proves $C_{\gamma} \in N$. And another use of Lemma 8.7 shows that $N$ also

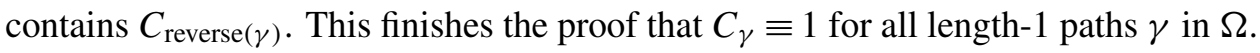

It remains only to show $D_{\gamma, k} \equiv 1$ for every length-0 path $\gamma$ in $\Omega$ and each $k \in I$ joined evenly to the unique point of $\gamma$, say $j$. Since $N$ contains $C_{\delta_{j}}$ and $D_{\delta_{j}, k}$ by definition, and $\delta_{j}$ followed by $\gamma$ is trivially equal to $\delta_{j}$, Lemma 8.3(ii) shows that $N$ contains $D_{\gamma, k}$ also.

We now review the general form of the description $F / N$ of $\mathfrak{G}_{2, \Omega}$ that we have just established. The generators are the $S_{i \in I}$ and the $X_{j \in \Omega}(t)$, with $t \in R$. The relations are the addition rules defining the $\mathfrak{U}_{j}$, the relations on the $S_{i}$ defining $\widehat{W}$, and the $B_{i \infty}, C_{z \in Z}, C_{\delta_{j}}$ and $D_{\delta_{j}, k}$, where $i$ varies over $I, j$ over $\Omega$, and $k \in I$ is evenly joined to $j$. The relations $B_{i \infty} \equiv 1$ say that $S_{i}^{2}$ centralizes or inverts every 
$X_{\infty}(t)$. Each relation $C_{z} \equiv 1$ says that a certain word in $\widehat{W}$ conjugates every $X_{\infty}(t)$ to itself. The relations $D_{\delta_{j}, k} \equiv 1$ say that certain other words in $\widehat{W}$ also commute with every $X_{\infty}(t)$. Finally, for each $j$, the relations $C_{\delta_{j}} \equiv 1$ express the $X_{j}(t)$ as conjugates of the $X_{\infty}(t)$ by still more words in $\widehat{W}$. The obvious way to simplify the presentation is to use this last batch of relations to eliminate the $X_{j \neq \infty}(t)$ from the presentation. We make this precise in the following lemma.

Lemma 8.9. Define $F_{\infty}=\mathfrak{U}_{\infty} * \widehat{W}$ and let $N_{\infty}$ be the subgroup normally generated by the $B_{i \infty}(i \in I)$, the $C_{z}(z \in Z)$, and the $D_{\delta_{j}, k}(j \in \Omega$ and $k \in I$ evenly joined $)$. Then the natural map $F_{\infty} / N_{\infty} \rightarrow F / N$ is an isomorphism.

Proof. We begin with the presentation $F / N$ from the previous paragraph and apply Tietze transformations. The relation $C_{\delta_{j}}(t) \equiv 1$ reads

$$
X_{j}(t) \equiv P_{\delta_{j}} X_{\infty}(t) P_{\delta_{j}}^{-1} .
$$

For $j=\infty$ this is the trivial relation $X_{\infty}(t)=X_{\infty}(t)$, which we may discard. For $j \neq \infty$ we use it to replace $X_{j}(t)$ by $P_{\delta_{j}} X_{\infty}(t) P_{\delta_{j}}^{-1}$ everywhere else in the presentation, and then discard $X_{j}(t)$ from the generators and $C_{\delta_{j}}(t)$ from the relators.

The only other occurrences of $X_{j \neq \infty}(t)$ in the presentation are in the relators defining $\mathfrak{U}_{j}$. After the replacement of the previous paragraph, these relations read

$$
P_{\delta_{j}} X_{\infty}(t) P_{\delta_{j}}^{-1} \cdot P_{\delta_{j}} X_{\infty}(u) P_{\delta_{j}}^{-1} \equiv P_{\delta_{j}} X_{\infty}(t+u) P_{\delta_{j}}^{-1} .
$$

These relations can be discarded because they are the $P_{\delta_{j}}$-conjugates of the relations $X_{\infty}(t) X_{\infty}(u) \equiv X_{\infty}(t+u)$. What remains is the presentation $F_{\infty} / N_{\infty}$.

Proof of Lemma 8.2. The previous lemma shows $\mathfrak{G}_{2, \Omega} \cong F_{\infty} / N_{\infty}$. So $\mathfrak{G}_{2, \Omega}$ is the quotient of $\mathfrak{U}_{\infty} * \widehat{W}$ by relations asserting that certain elements of $\widehat{W}$ act on $\mathfrak{U}_{\infty}$ by certain automorphisms. The relations $B_{i \infty}=1$ make $S_{i}^{2}$ act on $\mathfrak{U}_{\infty}$ by $(-1)^{A_{i \infty}}$. The relations $C_{z}=D_{\delta_{j}, k}=1$ make the words $P_{z}$ and $R_{\delta_{j}, k}$ centralize $\mathfrak{U}_{\infty}$.

By Lemma 7.2(iii), the $S_{i}^{2}$ generate the kernel of $\widehat{W} \rightarrow W$. By Theorem 5.7, the images of the $P_{z}$ and $R_{\delta_{j}, k}$ in $W$ generate the $W$-stabilizer of the simple root $\infty \in I$. Therefore, the $S_{i}^{2}, P_{z}$ and $R_{\delta_{j}, k}$ generate the $\widehat{W}$-stabilizer $\widehat{W}_{\infty}$ of $\infty$. Their actions on $\mathfrak{U}_{\infty}$ are the same as the ones given by the homomorphism $\widehat{W} \rightarrow W^{*}$, by Theorem 5.7. Therefore, $\mathfrak{G}_{2, \Omega}=\left(\mathfrak{U}_{\infty} \rtimes \widehat{W}_{\infty}\right) *_{\widehat{W}_{\infty}} \widehat{W}$. And Lemma 8.1 identifies this with $\left(*_{\alpha \in \Phi(\Omega)} \mathfrak{U}_{\alpha}\right) \rtimes \widehat{W}$, as desired.

\section{The isomorphism $\mathfrak{G}_{3} \cong \mathfrak{P S} \mathfrak{t}^{\text {Tits }} \rtimes \widehat{W}$}

We have two goals in this section. The first is to start from Theorem 7.5, that $\mathfrak{G}_{2} \cong\left(*_{\alpha \in \Phi} \mathfrak{U}_{\alpha}\right) \rtimes \widehat{W}$, and prove Theorem 7.11 , that $\mathfrak{G}_{3} \cong \mathfrak{P S t} \mathfrak{t}^{\text {Tits }} \rtimes \widehat{W}$. The second is to explain how one may discard many of the Chevalley relations; for example, for $E_{n \geq 6}$ one can get away with imposing the relations for a single unjoined 
pair of nodes of the Dynkin diagram, and for a single joined pair. The latter material is not necessary for our main results.

Proof of Theorem 7.11. First we show that the relators (7-10)-(7-23), regarded as elements of $\mathfrak{G}_{2} \cong\left(*_{\alpha \in \Phi} \mathfrak{U}_{\alpha}\right) \rtimes \widehat{W}$, become trivial in $\mathfrak{S t}^{\text {Tits }} \rtimes \widehat{W}$. Then we will show that they normally generate the whole kernel of $\mathfrak{G}_{2} \rightarrow \mathfrak{S t}^{\mathrm{Tits}} \rtimes \widehat{W}$.

If $\alpha, \beta$ are a prenilpotent pair of roots with $\theta(\alpha, \beta)=\{\alpha, \beta\}$, then the Chevalley relation for $\alpha$ and $\beta$ is $\left[\mathfrak{U}_{\alpha}, \mathfrak{U}_{\beta}\right]=1$. This shows that relators (7-10), (7-11), (7-13), (7-14), (7-17), (7-18) and (7-19) become trivial in $\mathfrak{S t}^{\text {Tits }} \rtimes \widehat{W}$. Careful calculation verifies that the remaining relators are equivalent to those given by Demazure in [SGA $3_{\text {III }}$ 1970, Exposé XXIII]. Here are some remarks on the correspondence between his notation and ours. In the $A_{2}$ case (his Proposition 3.2.1), his $\alpha$ and $\beta$ correspond to our $\alpha_{j}$ and $\alpha_{i}$, his $X_{\alpha}$ and $X_{\beta}$ to our $e_{j}$ and $e_{i}$, his $X_{-\alpha}$ and $X_{-\beta}$ to our $-f_{j}$ and $-f_{i}$, and his $p_{\alpha}(t)$ and $p_{\beta}(t)$ to our $X_{j}(t)$ and $X_{i}(t)$. His $w_{\alpha}$ and $w_{\beta}$ are not the same as our $S_{j}$ and $S_{i}$ (which are not even elements of $*_{\gamma \in \Phi} \mathfrak{U}_{\gamma}$ ), but their actions on the $\mathfrak{U}_{\gamma}$ are the same, so his $p_{\alpha+\beta}(t):=w_{\beta} p_{\alpha}(t) w_{\beta}^{-1}$ corresponds to our $S_{i} X_{j}(t) S_{i}^{-1}$. One can now check that our (7-12) is equivalent to his Proposition 3.2.1(iii).

In the $B_{2}$ case (his Proposition 3.3.1), his $\alpha$ and $\beta$ correspond to our $\alpha_{s}$ and $\alpha_{l}$, his $X_{\alpha}$ and $X_{\beta}$ to our $e_{s}$ and $e_{l}$, his $X_{-\alpha}$ and $X_{-\beta}$ to our $-f_{s}$ and $-f_{l}$, and his $p_{\alpha}(t)$ and $p_{\beta}(t)$ to our $X_{s}(t)$ and $X_{l}(t)$. His $w_{\alpha}$ and $w_{\beta}$ correspond to our $S_{s}$ and $S_{l}$ in the same sense as above. It follows that his $p_{\alpha+\beta}(t)$ and $p_{2 \alpha+\beta}(t)$ correspond to our $S_{l} X_{s}(t) S_{l}^{-1}$ and $S_{s} X_{l}(t) S_{s}^{-1}$. Then our (7-15) and (7-16) are equivalent to his Proposition 3.3.1. The $G_{2}$ case is the same (his Proposition 3.4.1), except that his $p_{\alpha+\beta}(t), p_{2 \alpha+\beta}(t), p_{3 \alpha+\beta}(t)$ and $p_{3 \alpha+2 \beta}(t)$ correspond to our

$$
S_{l} X_{S}(t) S_{l}^{-1}, \quad S_{s} S_{l} X_{s}(t) S_{l}^{-1} S_{s}^{-1}, \quad S_{s} X_{l}(-t) S_{s}^{-1} \quad \text { and } \quad S_{l} S_{s} X_{l}(-t) S_{s}^{-1} S_{l}^{-1} .
$$

Then our (7-20)-(7-23) are among the relations in his Proposition 3.4.1(iii).

As a check (indeed a second proof that our relations are the Chevalley relations) we constructed our elements of the various root groups in explicit representations of the Chevalley groups $\mathrm{SL}_{2} \times \mathrm{SL}_{2}, \mathrm{SL}_{3}, \mathrm{Sp}_{4}$ and $G_{2}$ over $R=\mathbb{Z}[t, u]$, faithful on the unipotent subgroups of their Borel subgroups. As mentioned in Remark 7.15, we used a computer to check that our relators map to the identity. By functoriality, the same holds with $R$ replaced by any ring. In addition to our relations, the root groups satisfy the Chevalley relations, by construction. By the isomorphism $\mathfrak{U}_{\theta(\alpha, \beta)} \cong \prod_{\gamma \in \theta(\alpha, \beta)} \mathfrak{U}_{\gamma}$ of underlying schemes (Lemma 6.1), the only relations having the form of the Chevalley relations that can hold are the Chevalley relations themselves. So our relations are among them.

It remains to prove that the Chevalley relators of any classically prenilpotent pair $\alpha^{\prime}, \beta^{\prime} \in \Phi$ become trivial in $\mathfrak{G}_{3}$. By classical prenilpotency, $\Phi_{0}^{\prime}:=\left(\mathbb{Q} \alpha^{\prime}+\mathbb{Q} \beta^{\prime}\right) \cap \Phi$ 
is an $A_{1}, A_{1}^{2}, A_{2}, B_{2}$ or $G_{2}$ root system. In the $A_{1}$ case we have $\alpha^{\prime}=\beta^{\prime}$ and the Chevalley relations amount to the commutativity of $\mathfrak{U}_{\alpha^{\prime}}$. This follows from $\mathfrak{U}_{\alpha^{\prime}} \cong R$. So we consider the other cases. There exists $w \in W$ sending $\Phi_{0}^{\prime}$ to the root system $\Phi_{0} \subseteq \Phi$ generated by some pair of simple roots. (Choose simple roots for $\Phi_{0}^{\prime}$. Then choose a chamber in the Tits cone which has two of its facets lying in the mirrors of those roots, and which lies on the positive sides of these mirrors. Choose $w$ to send this chamber to the standard one.)

We choose a pair of roots $\alpha, \beta \in \Phi_{0}$ as follows. First, they should have the same relative configuration as $\alpha^{\prime}, \beta^{\prime}$ have. (That is, they should have the same short/long root status, and make the same angle.) And second, their Chevalley relators should appear among (7-10)-(7-23). Such $\alpha, \beta$ can always be chosen. For example, in the $G_{2}$ case, (7-17)-(7-23) are, respectively, the Chevalley relations for two long roots with angle $\pi / 3$, a short and a long root with angle $\pi / 6$, two orthogonal roots, two long roots with angle $2 \pi / 3$, two short roots with angle $\pi / 3$, two short roots with angle $2 \pi / 3$, and a short and a long root with angle $5 \pi / 6$. The other cases are similarly exhaustive. By refining the choice of $w$, we may suppose that it sends $\left\{\alpha^{\prime}, \beta^{\prime}\right\}$ to $\{\alpha, \beta\}$. Now choose $\hat{w} \in \widehat{W}$ lying over $w$. The Chevalley relators for $\alpha^{\prime}, \beta^{\prime}$ are the $\hat{w}^{-1}$-conjugates of the Chevalley relators for $\alpha, \beta$. Since the latter become trivial in $\mathfrak{G}_{3}$, so do the former.

The proof of Theorem 7.11 exploited the $\widehat{W}$-action on $*_{\alpha \in \Phi} \mathfrak{U}_{\alpha}$ to obtain the Chevalley relators for all classically prenilpotent pairs from those listed explicitly in (7-10)-(7-23). One can further exploit this idea to omit many of the relators coming from the cases $m_{i j}=2$ or 3 . Our method derives from the notion of an ordered pair of simple roots being associate to another pair, due to [Brink and Howlett 1999] and [Borcherds 1998]. But we need very little of their machinery, so we will argue directly. There does not seem to be any similar simplification possible if $m_{i j}=4$ or 6 .

Proposition 9.1. Suppose $i, j, k \in I$ form an $A_{1} A_{2}$ diagram, with $j$ and $k$ joined. Then imposing the relation $\left[\mathfrak{U}_{i}, \mathfrak{U}_{j}\right]=1$ on $\mathfrak{G}_{2} \cong\left(*_{\alpha \in \Phi} \mathfrak{U}_{\alpha}\right) \rtimes \widehat{W}$ also imposes $\left[\mathfrak{U}_{i}, \mathfrak{U}_{k}\right]=1$. More formally, the normal closure of the relators (7-10) in $\mathfrak{G}_{2}$ contains the relators got from them by replacing $j$ by $k$.

Proof. Some element of the copy of $W\left(A_{2}\right)$ generated by $s_{j}$ and $s_{k}$ sends $\alpha_{j}$ to $\alpha_{k}$, and of course it fixes $\alpha_{i}$. Choose any lift of it to $\widehat{W}$. Conjugation by it in $\mathfrak{G}_{2}$ fixes $\mathfrak{U}_{i}$ and sends $\mathfrak{U}_{j}$ to $\mathfrak{U}_{k}$. So it sends the relators (7-10) to the relators got from them by replacing $j$ by $k$.

The lemma shows that imposing on $\mathfrak{G}_{2}$ the relations (7-10) for a few well-chosen unordered pairs $\{i, j\}$ in $I$ with $m_{i j}=2$ automatically imposes the corresponding relations for all such pairs. As examples, for spherical Dynkin diagrams it suffices 
to impose these relations for

$$
\begin{cases}3 \text { such pairs (that is, all of them) } & \text { for } D_{4}, \\ 2 \text { such pairs } & \text { for } B_{n \geq 4}, C_{n \geq 4} \text { or } D_{n \geq 5}, \\ 1 \text { such pair } & \text { for } A_{n \geq 3}, B_{3}, C_{3}, E_{n} \text { or } F_{4} .\end{cases}
$$

Proposition 9.2. Suppose $i, j, k \in I$ form an $A_{3}$ diagram, with $i$ and $k$ unjoined. Then the normal closure of the relators (7-11)-(7-12) in $\mathfrak{G}_{2} \cong\left(*_{\alpha \in \Phi} \mathfrak{U}_{\alpha}\right) \rtimes \widehat{W}$ contains the relators got from them by replacing $i$ and $j$ by $j$ and $k$, respectively.

Proof. The argument is the same as for Proposition 9.1, using an element of $W\left(A_{3}\right)$ that sends $\alpha_{i}$ and $\alpha_{j}$ to $\alpha_{j}$ and $\alpha_{k}$. An example of such an element is the "fundamental element" (or "long word") of $\left\langle s_{i}, s_{j}\right\rangle$, followed by the fundamental element of $\left\langle s_{i}, s_{j}, s_{k}\right\rangle$. The first transformation sends $\alpha_{i}$ and $\alpha_{j}$ to $-\alpha_{j}$ and $-\alpha_{i}$. The second sends $\alpha_{i}, \alpha_{j}$ and $\alpha_{k}$ to $-\alpha_{k},-\alpha_{j}$ and $-\alpha_{i}$.

Similarly to the $m_{i j}=2$ case, imposing on $\mathfrak{G}_{2}$ the relations (7-11)-(7-12) for some well-chosen ordered pairs $(i, j)$ in $I$ with $m_{i j}=3$ automatically imposes the corresponding relations for all such pairs. For spherical diagrams, it suffices to impose these relations for

$$
\begin{cases}4 \text { such pairs (that is, all of them) } & \text { for } F_{4}, \\ 2 \text { such pairs } & \text { for } A_{n \geq 2}, B_{n \geq 3} \text { or } C_{n \geq 3}, \\ 1 \text { such pair } & \text { for } D_{n \geq 4} \text { or } E_{n} .\end{cases}
$$

\section{The adjoint representation}

A priori, it is conceivable that for some commutative ring $R \neq 0$ and some generalized Cartan matrix $A$, the Steinberg group $\mathfrak{S t}_{A}(R)$ might collapse to the trivial group. That this doesn't happen follows from work of Tits [1987, §4] and Rémy [2002, Chapter 9] on the "adjoint representation" of $\mathfrak{S} \mathfrak{t}_{A}$. We will improve their results slightly by proving that the unipotent group scheme $\mathfrak{U}_{\Psi}$ embeds in the Steinberg group functor $\mathfrak{S t}_{A}$, for any nilpotent set of roots $\Psi$. We need this result in the next section, in our proof that $\mathfrak{P S t}_{A}(R) \rightarrow \mathfrak{S t}_{A}(R)$ is often an isomorphism.

Recall that Lemma 6.1 associates to $\Psi$ a unipotent group scheme $\mathfrak{U}_{\Psi}$ over $\mathbb{Z}$. Furthermore, there are natural homomorphisms $\mathfrak{U}_{\gamma} \rightarrow \mathfrak{U}_{\Psi}$ for all $\gamma \in \Psi$, and the product map $\prod_{\gamma \in \Psi} \mathfrak{U}_{\gamma} \rightarrow \mathfrak{U}_{\Psi}$ is an isomorphism of the underlying schemes, for any ordering of the factors.

Also in Section 6, we defined Tits' Steinberg functor $\mathfrak{S t}_{A}^{\text {Tits }}$ as the direct limit of the group schemes $\mathfrak{U}_{\gamma}$ and $\mathfrak{U}_{\Psi}$, where $\gamma$ varies over $\Phi$, and $\Psi$ varies over the nilpotent subsets of $\Phi$ of the form $\Psi=\theta(\alpha, \beta)$, with $\alpha, \beta$ a prenilpotent pair of roots. Composing with $\mathfrak{S t}_{A}^{\text {Tits }} \rightarrow \mathfrak{S t}_{A}$, we have natural maps $\mathfrak{U}_{\Psi} \rightarrow \mathfrak{S t}_{A}$ for such $\Psi$. A special case of the following theorem is that these maps are embeddings. We would like to say that the same holds for $\Psi$ an arbitrary nilpotent set of roots. But 
"the same holds" doesn't quite have meaning, because the definition of $\mathfrak{S t}_{A}$ doesn't provide a natural map $\mathfrak{U}_{\Psi} \rightarrow \mathfrak{S t}_{A}$ for general $\Psi$. So we phrase the result as follows.

Theorem 10.1 (injection of unipotent subgroups into $\mathfrak{S t}_{A}$ ). Suppose A is a generalized Cartan matrix and $\Psi$ is a nilpotent set of roots. Then there is a unique homomorphism $\mathfrak{U}_{\Psi} \rightarrow \mathfrak{S t}_{A}$ whose restriction to each $\mathfrak{U}_{\alpha \in \Psi}$ is the natural map to $\mathfrak{S t}_{A}$, and it is an embedding.

Uniqueness is trivial, by the isomorphism of underlying schemes $\mathfrak{U}_{\Psi} \cong \prod_{\alpha \in \Psi} \mathfrak{U}_{\alpha}$. Existence is easy: every pair of roots in $\Psi$ is prenilpotent, their Chevalley relations hold in $\mathfrak{S t}$, and these relations suffice to define $\mathfrak{U}_{\Psi}$ as a quotient of $*_{\beta \in \Psi} \mathfrak{U}_{\beta}$. So we must show that this homomorphism is an embedding. Our proof below relies on a linear representation of $\mathfrak{S t}_{A}$, functorial in $R$, called the adjoint representation. Its essential properties are developed in [Rémy 2002, Chapter 9], relying on a $\mathbb{Z}$-form of the universal enveloping algebra of $\mathfrak{g}$ introduced in [Tits 1987, §4].

Following Tits and Rémy we will indicate all ground rings other than $\mathbb{Z}$ explicitly, in particular writing $\mathfrak{g}_{\mathbb{C}}$ for the Kac-Moody algebra $\mathfrak{g}$. We write $\mathcal{U}_{\mathbb{C}}$ for its universal enveloping algebra. Recall from Section 6 that for each root $\alpha \in \Phi$ we distinguished a subgroup $\mathfrak{g}_{\alpha, \mathbb{Z}} \cong \mathbb{Z}$ of $\mathfrak{g}_{\alpha, \mathbb{C}}$ and the set $E_{\alpha}$ consisting of the two generators for $\mathfrak{g}_{\alpha, \mathbb{Z}}$.

Generalizing work of Kostant [1966] and Garland [1978], Tits defined an integral form of $\mathcal{U}_{\mathbb{C}}$, meaning a subring $\mathcal{U}$ with the property that the natural map $\mathcal{U} \otimes \mathbb{C} \rightarrow \mathcal{U}_{\mathbb{C}}$ is an isomorphism. It is the subring generated by the divided powers $e_{i}^{n} / n$ ! and $f_{i}^{n} / n$ !, as $i$ varies over $I$, together with the "binomial coefficients"

$$
\left(\begin{array}{l}
h \\
n
\end{array}\right):=h(h-1) \cdots(h-n+1) / n !,
$$

where $h$ varies over the $\mathbb{Z}$-submodule of $\mathfrak{g}_{0, \mathbb{C}}$ with basis $\bar{h}_{i}$.

Remark 10.2 (the role of the root datum). Although it isn't strictly necessary, we mention that lurking behind the scenes is a choice of root datum. It is the one which Rémy calls simply connected [2002, §7.1.2] and Tits calls "simply connected in the strong sense" [1987, Remark 3.7(c)]. A choice of root datum is necessary to define $\mathcal{U}$, hence the adjoint representation, and the choice does matter. For example, $\mathrm{SL}_{2}$ and $\mathrm{PGL}_{2}$ have the same Cartan matrix, but different root data. Their adjoint representations are distinct in characteristic 2 , when we compare them by regarding both as representations of $\mathrm{SL}_{2}$ via the central isogeny $\mathrm{SL}_{2} \rightarrow \mathrm{PGL}_{2}$. Similarly, they provide distinct representations of $\mathfrak{S t}_{A_{1}}$. For us the essential fact is that each $\bar{h}_{i}$ generates a $\mathbb{Z}$-module summand of $\mathcal{U}$, as explained in the next paragraph. As an example of what could go wrong, using the root datum for $\mathrm{PGL}_{2}$ would lead to $\bar{h}_{i} / 2 \in \mathcal{U}$ and spoil the proof of Theorem 10.1 in characteristic 2 .

In the sense Tits used, an integral form of a $\mathbb{C}$-algebra need not be free as a $\mathbb{Z}$-module. For example, $\mathbb{Q}$ is a $\mathbb{Z}$-form of $\mathbb{C}$ since $\mathbb{Q} \otimes_{\mathbb{Z}} \mathbb{C} \rightarrow \mathbb{C}$ is an isomorphism. 
But $\mathcal{U}$ is free as a $\mathbb{Z}$-module. To see this, one uses the following ingredients from [Tits $1987, \S 4.4]$. First, the $\mathbb{Z}^{I}$-grading makes it easy to see that

$$
\mathcal{U}_{+}:=\left\langle\left\{e_{i}^{n} / n ! \mid i \in I \text { and } n \geq 0\right\}\right\rangle \text { and } \mathcal{U}_{-}:=\left\langle\left\{f_{i}^{n} / n ! \mid i \in I \text { and } n \geq 0\right\}\right\rangle
$$

are free as $\mathbb{Z}$-modules, and that $\left\{e_{i \in I}\right\}$ and $\left\{f_{i \in I}\right\}$ extend to bases of them. Second, the universal enveloping algebra $\mathcal{U}_{0, \mathbb{C}}$ of the Cartan algebra $\mathfrak{g}_{0, \mathbb{C}}$ is a polynomial ring. This makes it easy to see that

$$
\mathcal{U}_{0}:=\left\langle\left\{\left(\begin{array}{l}
h \\
n
\end{array}\right) \mid h \in \bigoplus_{i} \mathbb{Z} \bar{h}_{i} \text { and } n \geq 0\right\}\right\rangle
$$

is free as a $\mathbb{Z}$-module. Indeed, Proposition 2 of [Bourbaki 1975, Chapter VIII, §12.4] extends $\left\{\bar{h}_{i \in I}\right\}$ to a $\mathbb{Z}$-basis for $\mathcal{U}_{0}$. Finally, $\mathcal{U}_{-} \otimes \mathcal{U}_{0} \otimes \mathcal{U}_{+} \rightarrow \mathcal{U}$ is an isomorphism by [Tits 1987 , Proposition 2]. One can obtain a $\mathbb{Z}$-basis for $\mathcal{U}$ by tensoring together members of bases for $\mathcal{U}_{-}, \mathcal{U}_{0}$ and $\mathcal{U}_{+}$.

A key property of $\mathcal{U}$ is its stability under $\left(\operatorname{ad} e_{i}\right)^{n} / n$ ! and $\left(\operatorname{ad} f_{i}\right)^{n} / n$ ! for all $n \geq 0$ (see [Tits 1987, equation (12)]). The local nilpotence of ad $e_{i}$ and ad $f_{i}$ on $\mathfrak{g}_{\mathbb{C}}$ implies their local nilpotence on $\mathcal{U}_{\mathbb{C}}$. As exponentials of locally nilpotent derivations, $\exp$ ad $e_{i}$ and exp ad $f_{i}$ are automorphisms of $\mathcal{U}_{\mathbb{C}}$. Since they preserve its subring $\mathcal{U}$, they are automorphisms of it. Since the generators $s_{i}^{*}$ for $W^{*}$ are defined in terms of them by (5-1), $W^{*}$ also acts on $\mathcal{U}$.

Because $\mathcal{U}$ is free as a $\mathbb{Z}$-module, $\mathcal{U}_{R}:=\mathcal{U} \otimes R$ is free as an $R$-module. It is the $R$-module underlying the adjoint representation of $\mathfrak{S t}_{A}(R)$ in Theorem 10.3 below, which we will now develop. For each root $\alpha$ we define an exponential map $\exp : \mathfrak{U}_{\alpha}(R) \rightarrow \operatorname{Aut}\left(\mathcal{U}_{R}\right)$ as follows. Recall that $\mathfrak{U}_{\alpha}(R)$ was defined as $\mathfrak{g}_{\alpha, \mathbb{Z}} \otimes R$. If $x$ is an element of this, then we choose $e \in E_{\alpha}$ and define $t \in R$ by $x=t e$. Then we define $\exp x$ to be the $R$-module endomorphism of $\mathcal{U}_{R}$ given by $\sum_{n=0}^{\infty} t^{n}(\operatorname{ad} e)^{n} / n !$. The apparent dependence on the choice of $e$ is no dependence at all, because if one makes the other choice $-e$ then one must also replace $t$ by $-t$. As shown in [Rémy $2002, \S 9.4]$, exp $x$ is an $R$-algebra automorphism of $\mathcal{U}_{R}$, not merely an $R$-module endomorphism.

Theorem 10.3 (adjoint representation). For any commutative ring $R$, there exists $a$ homomorphism $\mathrm{Ad}: \mathfrak{S t}_{A}(R) \rightarrow$ Aut $\mathcal{U}_{R}$, functorial in $R$ and characterized by the following property. For every root $\alpha$ the exponential map exp : $\mathfrak{U}_{\alpha}(R) \rightarrow \operatorname{Aut} \mathcal{U}_{R}$ factors as the natural map $\mathfrak{U}_{\alpha}(R) \rightarrow \mathfrak{S t}_{A}(R)$ followed by Ad.

Proof. This is from Sections 9.5.2-9.5.3 of [Rémy 2002]. We remark that he used Tits' version of the Steinberg functor (what we call $\mathfrak{S t}_{A}^{\text {Tits }}$ ) rather than the Morita-Rehmann version (what we call $\mathfrak{S t}_{A}$ ). But his Theorem 9.5.2 states that Ad is a representation of Tits' Kac-Moody group $\tilde{\mathcal{G}}_{D}(R)$. Since the extra relations in the Morita-Rehmann version of the Steinberg group are among those defining $\tilde{\mathcal{G}}_{D}(R)$, we may regard Ad as a representation of $\mathfrak{S t}_{A}(R)$. 
A few comments are required to identify our relations with (some of) his. $\tilde{\mathcal{G}}_{D}(R)$ is defined in [Rémy 2002, §8.3.3] as a quotient of the free product of $\mathfrak{P S t}_{A}^{\text {Tits }}(R)$ with a certain torus $\mathcal{T}$. Rémy's third relation identifies our $\tilde{h}_{i}(r)$ from (6-3) with the element of $\mathcal{T}$ that Rémy calls $r^{h_{i}}$. Rémy's first relation says how $\mathcal{T}$ acts on each $\mathfrak{U}_{j}$, and amounts to our (6-8). Rémy's fourth relation is our (6-10), saying that each $\tilde{s}_{i}$ acts as $s_{i}^{*}$ on every $\mathfrak{U}_{\beta}$. Rémy's second relation says how each $\tilde{s}_{i}$ acts on $\mathcal{T}$, and in particular describes $\tilde{s}_{j} r^{h_{i}} \tilde{s}_{j}^{-1}$. Together with the known action of $\tilde{h}_{i}(r)$ on $\mathfrak{U}_{j}$ and the fact that $\tilde{s}_{j}$ exchanges $\mathfrak{U}_{ \pm j}$, this describes how $\tilde{h}_{i}(r)$ acts on $\mathfrak{U}_{-j}$, and recovers our relation (6-9). By Theorem 6.4, this shows that all the relations in our $\mathfrak{S t}(R)$ hold in $\tilde{\mathcal{G}}_{D}(R)$.

Proof of Theorem 10.1. By induction on $|\Psi|$. The base case, with $\Psi=\varnothing$, is trivial. So suppose $|\Psi|>0$. Since $\Psi$ is nilpotent, there is some chamber pairing positively with every member of $\Psi$ and another one pairing negatively with every member. It follows that there is a chamber pairing positively with one member and negatively with all the others. In other words, after applying an element of $W^{*}$ we may suppose that $\Psi$ contains exactly one positive root. We may even suppose that this root is simple, say $\alpha_{i}$. Write $\Psi_{0}$ for $\Psi-\left\{\alpha_{i}\right\}$.

Consider the adjoint representation $\mathfrak{U}_{\Psi}(R) \rightarrow \mathfrak{S t}(R) \rightarrow$ Aut $\mathcal{U}_{R}$, in particular the action of $x \in \mathfrak{U}_{\Psi}(R)$ on $f_{i} \in \mathcal{U}_{R}$. If $x \in \mathfrak{U}_{\Psi_{0}}(R)$ then the component of $x\left(f_{i}\right)$ in the subspace of $\mathcal{U}_{R}$ graded by $0 \in \mathbb{Z}^{I}$ is trivial, since $f_{i}$ and the $\beta \in \Psi_{0}$ are all negative roots. On the other hand, we can work out the action of $\mathfrak{x}_{i}(t)$ as follows. A computation in $\mathcal{U}$ shows

$$
\left(\operatorname{ad} e_{i}\right)\left(f_{i}\right)=-\bar{h}_{i}, \quad \frac{1}{2}\left(\operatorname{ad} e_{i}\right)^{2}\left(f_{i}\right)=e_{i}, \quad \text { and } \quad \frac{1}{n !}\left(\operatorname{ad} e_{i}\right)^{n}\left(f_{i}\right)=0
$$

for $n>2$. Therefore, we have

$$
\operatorname{Ad}\left(\mathfrak{x}_{i}(t)\right)\left(f_{i}\right)=\sum_{n=0}^{\infty} t^{n} \frac{\left(\operatorname{ad} e_{i}\right)^{n}}{n !}\left(f_{i}\right)=f_{i}-t \bar{h}_{i}+t^{2} e_{i} .
$$

Recall that $f_{i}, \bar{h}_{i}$ and $e_{i}$ are three members of a $\mathbb{Z}$-basis for $\mathcal{U}$. So their images in $\mathcal{U}_{R}$ are members of an $R$-basis. If $t \neq 0$ then the component of $\operatorname{Ad}\left(\mathfrak{x}_{i}(t)\right)\left(f_{i}\right)$ graded by $0 \in \mathbb{Z}^{I}$ is the nonzero element $-t \bar{h}_{i}$ of $\mathcal{U}_{R}$.

Therefore, only the trivial element of $\mathfrak{U}_{i}(R)$ maps into the image of $\mathfrak{U}_{\Psi_{0}}(R)$ in Aut $\mathcal{U}_{R}$. So the same is true with $\mathfrak{S t}(R)$ in place of Aut $\mathcal{U}_{R}$. From induction and the bijectivity of the product map $\mathfrak{U}_{i}(R) \times \mathfrak{U}_{\Psi_{0}}(R) \rightarrow \mathfrak{U}_{\Psi}(R)$ it follows that $\mathfrak{U}_{\Psi}(R)$ embeds in $\mathfrak{S t}(R)$.

\section{1. $\mathfrak{P S} \mathfrak{t} \rightarrow \mathfrak{S} \mathfrak{t}$ is often an isomorphism}

The purpose of this section is to prove parts (iii)-(iv) of Theorem 1.1, showing that the natural map $\mathfrak{P S t}_{A}(R) \rightarrow \mathfrak{S t}_{A}(R)$ is an isomorphism for many choices of 
generalized Cartan matrix $A$ and commutative ring $R$. These cases include most of part (ii) of the same theorem; see [Allcock 2016] for the complete result. And part (i) of the theorem is the case that $A$ is spherical. As remarked in Section 7, in this case $\mathfrak{P S t}_{A}$ and $\mathfrak{S t}_{A}$ are the same group by definition.

In the case that $R$ is a field, Abramenko and Mühlherr [1997] proved our (iv) with Kac-Moody groups in place of Steinberg groups. Our proof of (iv) derives from the proof of their Theorem A; with the following preparatory lemma, the argument goes through in our setting. For (iii) we use a more elaborate form of the idea, with Lemma 11.2 as preparation.

Lemma 11.1 (generators for unipotent groups in rank 2). Let $R$ be a commutative ring, $\Phi$ be a rank-2 spherical root system equipped with a choice of simple roots, and $\Phi^{+}$be the set of positive roots. If $\Phi$ has type $A_{1}^{2}$ or $A_{2}$ then $\mathfrak{U}_{\Phi^{+}}(R)$ is generated by the root groups of the simple roots.

If $\Phi$ has type $B_{2}$ then write $\alpha_{s}$ and $\alpha_{l}$ for the short and long simple roots, and $\alpha_{s^{\prime}}\left(\right.$ resp. $\left.\alpha_{l^{\prime}}\right)$ for the image of $\alpha_{s}$ (resp. $\left.\alpha_{l}\right)$ under reflection in $\alpha_{l}\left(\right.$ resp. $\left.\alpha_{s}\right)$. Then $\mathfrak{U}_{\Phi^{+}}(R)$ is generated by $\mathfrak{U}_{s}(R), \mathfrak{U}_{l}(R)$ and either one of $\mathfrak{U}_{s^{\prime}}(R)$ and $\mathfrak{U}_{l^{\prime}}(R)$. If $R$ has no quotient $\mathbb{F}_{2}$ then $\mathfrak{U}_{s}(R)$ and $\mathfrak{U}_{l}(R)$ suffice.

If $\Phi$ has type $G_{2}$ then, using notation as for $B_{2}, \mathfrak{U}_{\Phi^{+}}(R)$ is generated by $\mathfrak{U}_{s}(R)$, $\mathfrak{U}_{l}(R)$ and $\mathfrak{U}_{s^{\prime}}(R)$. If $R$ has no quotient $\mathbb{F}_{2}$ or $\mathbb{F}_{3}$ then $\mathfrak{U}_{s}(R)$ and $\mathfrak{U}_{l}(R)$ suffice.

Proof. We will suppress the dependence of group functors on $R$, always meaning groups of points over $R$. The $A_{1}^{2}$ case is trivial because the simple roots are the only positive roots.

In the $A_{2}$ case we write $\alpha_{i}$ and $\alpha_{j}$ for the simple roots. The only other positive root is $\alpha_{i}+\alpha_{j}$. As in Section 6, we choose $e_{i} \in E_{i}$ and $e_{j} \in E_{j}$. Then we can use the notation $X_{i}(t), X_{j}(t)$ for the elements of $\mathfrak{U}_{i}$ and $\mathfrak{U}_{j}$, where $t$ varies over $R$. The Chevalley relation (7-12) is $\left[X_{i}(t), X_{j}(u)\right]=S_{i} X_{j}(t u) S_{i}^{-1}$. Therefore, every element of $S_{i} \mathfrak{U}_{j}(R) S_{i}^{-1}$ lies in $\left\langle\mathfrak{U}_{i}(R), \mathfrak{U}_{j}(R)\right\rangle$. Since $S_{i} \mathfrak{U}_{j} S_{i}^{-1}=\mathfrak{U}_{\alpha_{i}+\alpha_{j}}$, the proof is complete.

In the $B_{2}$ and $G_{2}$ cases we choose $e_{s} \in E_{s}$ and $e_{l} \in E_{l}$, so we may speak of $X_{s}(t) \in \mathfrak{U}_{s}$ and $X_{l}(u) \in \mathfrak{U}_{l}$. We write $X_{s^{\prime}}(t)$ for $S_{l} X_{s}(t) S_{l}^{-1}$ and $X_{l^{\prime}}(t)$ for $S_{s} X_{l}(t) S_{s}^{-1}$. In the $G_{2}$ case we also define

$$
X_{s^{\prime \prime}}(t)=S_{s} S_{l} X_{s}(t) S_{l}^{-1} S_{s}^{-1} \quad \text { and } \quad X_{l^{\prime \prime}}(t)=S_{l} S_{s} X_{l}(t) S_{s}^{-1} S_{l}^{-1} .
$$

Rather than mimicking the direct computation of the $A_{2}$ case, we use the wellknown fact that a subset of a nilpotent group generates that group if and only if its image in the abelianization generates the abelianization. We will apply this to the subgroup of $\mathfrak{U}_{\Phi^{+}}$generated by $\mathfrak{U}_{s} \cup \mathfrak{U}_{l}$. Namely, we write $Q$ for the quotient of the abelianization of $\mathfrak{U}_{\Phi^{+}}$by the image of $\left\langle\mathfrak{U}_{s}, \mathfrak{U}_{l}\right\rangle$. Under the hypotheses about $R$ having no tiny fields as quotients, we will prove $Q=0$. In this case it follows that 
$\left\langle\mathfrak{U}_{s}, \mathfrak{U}_{l}\right\rangle$ maps onto the abelianization and is therefore all of $\mathfrak{U}_{\Phi^{+}}$. We must also prove, this time with no hypotheses on $R$, that $\mathfrak{U}_{\Phi^{+}}=\left\langle\mathfrak{U}_{s}, \mathfrak{U}_{l}, \mathfrak{U}_{s^{\prime}}\right\rangle$ and (in the $B_{2}$ case) that $\mathfrak{U}_{\Phi^{+}}=\left\langle\mathfrak{U}_{s}, \mathfrak{U}_{l}, \mathfrak{U}_{l^{\prime}}\right\rangle$. This uses the same argument, with calculations so much simpler that we omit them.

First consider the $B_{2}$ case. Among the Chevalley relators defining $\mathfrak{U}_{\Phi^{+}}$are (7-15) and (7-16), namely

$$
\begin{aligned}
& {\left[X_{S}(t), X_{s^{\prime}}(u)\right] \cdot X_{l^{\prime}}(2 t u)} \\
& {\left[X_{S}(t), X_{l}(u)\right] \cdot X_{l^{\prime}}\left(-t^{2} u\right) X_{s^{\prime}}(t u)}
\end{aligned}
$$

for all $t, u \in R$. The remaining Chevalley relations say that various root groups commute with various other root groups. Therefore, the abelianization of $\mathfrak{U}_{\Phi^{+}}$is the quotient of the abelian group

$$
\mathfrak{U}_{s} \times \mathfrak{U}_{l} \times \mathfrak{U}_{s^{\prime}} \times \mathfrak{U}_{l^{\prime}} \cong R^{4}
$$

by the images of the displayed relators. We obtain $Q$ by killing the image of $\mathfrak{U}_{s} \times \mathfrak{U}_{l}$.

So, changing to additive notation, $Q$ is the quotient of $\mathfrak{U}_{s^{\prime}} \oplus \mathfrak{U}_{l^{\prime}} \cong R^{2}$ by the subgroup generated by $0 \oplus 2 R$ and all $\left(t u,-t^{2} u\right)$, where $t, u$ vary over $R$. Taking $t=1$ in the latter shows that $2 R \oplus 0$ also dies in $Q$. So $Q$ is the quotient of $(R / 2 R)^{2}$ by the subgroup generated by all $\left(t u,-t^{2} u\right)$. That is, $Q$ is (the abelian group underlying) the quotient of $(R / 2 R)^{2}$ by the submodule(!) generated by all $\left(t,-t^{2}\right)$. This submodule contains $(1,-1)$, so it is equally well-generated by it and all $\left(t,-t^{2}\right)-t(1,-1)=\left(0, t-t^{2}\right)$. We may discard the first summand $R / 2 R$ from the generators and $(1,-1)$ from the relators. So $Q$ is the (abelian group underlying) the quotient of $R / 2 R$ by the ideal $I$ generated by all $t-t^{2}$. To prove $Q=0$ we will suppose $Q \neq 0$ and derive a contradiction. As a nonzero ring with identity, $R / I$ has some field as a quotient, in which $t=t^{2}$ holds identically. The only field with this property is $\mathbb{F}_{2}$, which is a contradiction since we supposed that $R$ has no such quotient.

For the $G_{2}$ case the Chevalley relators include

$$
\begin{aligned}
& {\left[X_{l}(t), X_{l^{\prime}}(u)\right] \cdot X_{l^{\prime \prime}}(-t u)} \\
& {\left[X_{s}(t), X_{s^{\prime \prime}}(u)\right] \cdot X_{l^{\prime}}(-3 t u)} \\
& {\left[X_{s}(t), X_{s^{\prime}}(u)\right] \cdot X_{l^{\prime \prime}}\left(3 t u^{2}\right) X_{l^{\prime}}\left(3 t^{2} u\right) X_{s^{\prime \prime}}(2 t u)} \\
& {\left[X_{s}(t), X_{l}(u)\right] \cdot X_{l^{\prime \prime}}\left(t^{3} u^{2}\right) X_{l^{\prime}}\left(-t^{3} u\right) X_{s^{\prime}}(t u) X_{s^{\prime \prime}}\left(-t^{2} u\right)} \\
& {\left[X_{s^{\prime}}(t), X_{s^{\prime \prime}}(u)\right] \cdot X_{l^{\prime \prime}}(-3 t u)}
\end{aligned}
$$

for all $t, u \in R$. The first four relations are from (7-20)-(7-23). The fifth is the conjugate of (7-21) by $S_{l}$, which commutes with $\mathfrak{U}_{s^{\prime \prime}}$ and sends $X_{s}(t)$ to $X_{s^{\prime}}(t)$ and $X_{l^{\prime}}(-3 t u)$ to $X_{l^{\prime \prime}}(-3 t u)$, by their definitions. All the remaining Chevalley relations say that various root groups commute with each other. 
Proceeding as in the $B_{2}$ case, we see that $Q$ is the quotient of the abelian group $\mathfrak{U}_{l^{\prime}} \oplus \mathfrak{U}_{s^{\prime \prime}} \oplus \mathfrak{U}_{s^{\prime}} \oplus \mathfrak{U}_{l^{\prime \prime}}$ by the subgroup generated by the relators $(0,0,0,-t u)$, $(-3 t u, 0,0,0),\left(3 t^{2} u, 2 t u, 0,3 t u^{2}\right),\left(-t^{3} u,-t^{2} u, t u, t^{3} u^{2}\right)$ and $(0,0,0,-3 t u)$, where $t, u$ vary over $R$. Because of the first relator, we may discard the $\mathfrak{U}_{l^{\prime \prime}}$ summand. This leads to the following description of $Q$ : the quotient of $R^{3}$ by the $R$-submodule spanned by the relators $(-3 t, 0,0),\left(3 t^{2}, 2 t, 0\right)$ and $\left(-t^{3},-t^{2}, t\right)$, where $t$ varies over $R$. Using $(-1,-1,1)$ in the same way we used $(1,-1)$ in the $B_{2}$ case shows that $Q$ is the quotient of $R^{2}$ by the submodule generated by all $(-3 t, 0),\left(3 t^{2}, 2 t\right)$ and $\left(t^{3}-t, t^{2}-t\right)$. This is the same as the quotient of $R / 3 R \oplus R / 2 R$ by the submodule generated by all $\left(t^{3}-t, t^{2}-t\right)$. Now, $R / 3 R \oplus R / 2 R$ is isomorphic to $R / 6 R$ by $(a, b) \leftrightarrow 2 a+3 b$. So $Q$ is the quotient of $R / 6 R$ by the ideal $I$ generated by $2\left(t^{3}-t\right)+3\left(t^{2}-t\right)$ for all $t$. As in the $B_{2}$ case, if $Q \neq 0$ then it has a further quotient that is a field $F$, obviously of characteristic 2 or 3 . In $F$, either $t^{2}=t$ holds identically or $t^{3}=t$ holds identically, according to these two possibilities. So $F=\mathbb{F}_{2}$ or $\mathbb{F}_{3}$, a contradiction.

Lemma 11.2 (generators for unipotent groups in rank 3). Let $R$ be a commutative ring, $\Phi$ be a spherical root system of rank $3,\left\{\beta_{i \in I}\right\}$ be simple roots for it, and $\Phi^{+}$ be the corresponding set of positive roots. Write $s_{i}$ for the reflection in $\beta_{i}$, and for each ordered pair $(i, j)$ of distinct elements of I write $\gamma_{i, j}$ for $s_{i}\left(\beta_{j}\right)$. Then $\mathfrak{U}_{\Phi^{+}}(R)$ is generated by the $\mathfrak{U}_{\beta_{i}}(R)$ and the $\mathfrak{U}_{\gamma_{i, j}}(R)$.

Proof. As in the previous proof, we suppress the dependence of group functors on $R$. If $\Phi$ is reducible then we apply the previous lemma. So it suffices to treat the cases $\Phi=A_{3}, B_{3}$ and $C_{3}$. We write $U$ for the subgroup of $\mathfrak{U}_{\Phi^{+}}$generated by the $\mathfrak{U}_{\beta_{i}}$ and $\mathfrak{U}_{\gamma_{i, j}}$. We must show that it is all of $\mathfrak{U}_{\Phi^{+}}$.

For type $A_{3}$ we describe $\Phi$ by using four coordinates summing to zero, and take the simple roots $\beta_{i}$ to be $(+-00),(0+-0)$ and $(00+-)$, where \pm are short for \pm 1 . The $\gamma_{i, j}$ are the roots $(+0-0)$ and $(0+0-)$. The only remaining positive root is $(+00-)$. This is the sum of $(+0-0)$ and $(00+-)$. So the $A_{2}$ case of Lemma 11.1 shows that its root group lies in the $U$.

For type $B_{3}$ we take the simple roots $\beta_{i}$ to be $(+-0),(0+-)$ and $(00+)$. The $\gamma_{i, j}$ are $(+0-)$ and $(0+0)$. The remaining positive roots are $(+00),(++0),(+0+)$ and $(0++)$. First, $(00+),(0+0)$ and $(0+-)$ are three of the four positive roots of a $B_{2}$ root system in $\Phi$, including a pair of simple roots for it. Since $U$ contains $\mathfrak{U}_{00+}$, $\mathfrak{U}_{0+0}$ and $\mathfrak{U}_{0+-}$, Lemma 11.1 shows that $U$ also contains a root group corresponding to the fourth positive root, namely $(0++)$. Second, applying the $A_{2}$ case of that lemma to $\mathfrak{U}_{0++}, \mathfrak{U}_{+-0} \subseteq U$ shows that $U$ also contains $\mathfrak{U}_{+0+}$. Third, repeating this using $\mathfrak{U}_{+0+}, \mathfrak{U}_{0+-} \subseteq U$ shows that $U$ contains $\mathfrak{U}_{++0}$. Finally, using the $B_{2}$ case again, the fact that $U$ contains $\mathfrak{U}_{00+}, \mathfrak{U}_{+0-}$ and $\mathfrak{U}_{+0+}$ shows that $U$ contains $\mathfrak{U}_{+00}$. We have shown that $U$ contains all the positive root groups, so $U=\mathfrak{U}_{\Phi^{+}}$, as desired. 
The $C_{3}$ case is the same: replacing the short roots $(1,0,0),(0,1,0)$ and $(0,0,1)$ by $(2,0,0),(0,2,0)$ and $(0,0,2)$ does not affect the proof.

The next proof uses the geometric language of the Tits cone (or Coxeter complex), its subdivision into chambers, and the combinatorial distance between chambers. Here is minimal background; see [Rémy 2002, Chapter 5] for more. The root system $\Phi$ lies in $\mathbb{Z}^{I} \subseteq \mathbb{R}^{I}$. The fundamental (open) chamber is the set of elements in $\operatorname{Hom}\left(\mathbb{R}^{I}, \mathbb{R}\right)$ having positive pairing with all simple roots. We defined an action of the Weyl group $W$ on $\mathbb{Z}^{I}$ in Section 4 , so $W$ also acts on this dual space. A chamber means a $W$-translate of the fundamental chamber, and the Tits cone means the union of the closures of the chambers. It is tiled by them. W's action is properly discontinuous on the interior of this cone. A gallery of length $n$ means a sequence of chambers $C_{0}, \ldots, C_{n}$, each $C_{i}$ sharing a facet with $C_{i-1}$ for $i=1, \ldots, n$. The gallery is called minimal if there is no shorter gallery from $C_{0}$ to $C_{n}$.

To each root $\alpha \in \Phi$ corresponds a halfspace in the Tits cone, namely those points in it having positive pairing with $\alpha$. We write the boundary of this halfspace as $\alpha^{\perp}$. We will identify each root with its halfspace, so we may speak of roots containing chambers. In this language, a set of roots is prenilpotent if there is some chamber lying in all of them, and some chamber lying in none of them.

Proof of Theorem 1.1(iii)-(iv). We suppress the dependence of group functors on $R$, always meaning groups of points over $R$. Recall that $\mathfrak{S t}$ is obtained from $\mathfrak{P S t}$ by adjoining the Chevalley relations for the prenilpotent pairs of roots that are not classically prenilpotent. So we must show that these relations already hold in $\mathfrak{P S t}$. For $\Psi$ any nilpotent set of roots we will write $G_{\Psi}$ for the subgroup of $\mathfrak{P S t}$ generated by the $\mathfrak{U}_{\alpha \in \Psi}$. Theorem 10.1 shows that the subgroup of $\mathfrak{S t}$ generated by these $\mathfrak{U}_{\alpha}$ is a copy of $\mathfrak{U}_{\Psi}$, so we will just write $\mathfrak{U}_{\Psi}$ for it.

We will prove by induction the following assertion $\left(N_{n \geq 1}\right)$ : Suppose $C_{0}, \ldots, C_{n}$ is a minimal gallery, for each $k=1, \ldots, n$ let $\alpha_{k}$ be the root which contains $C_{k}$ but not $C_{k-1}$, and define $\Psi=\left\{\alpha_{1}, \ldots, \alpha_{n}\right\}$ and $\Psi_{0}=\Psi-\left\{\alpha_{n}\right\}$. Then $\mathfrak{U}_{\alpha_{n}}$ normalizes $G_{\Psi_{0}}$ in $G_{\Psi}$. (The $N$ stands for "normalizes". Also, it is easy to see that $\Psi$ is the set of all roots containing $C_{n}$ but not $C_{0}$, so it is nilpotent, and similarly for $\Psi_{0}$. So $G_{\Psi}$ and $G_{\Psi_{0}}$ are defined.)

Assuming $\left(N_{n}\right)$ for all $n \geq 1$, it follows that, for $\Psi$ of this form, the multiplication map $\mathfrak{U}_{\alpha_{1}} \times \cdots \times \mathfrak{U}_{\alpha_{n}} \rightarrow G_{\Psi}$ in $\mathfrak{P S t}$ is surjective. We know from Lemma 6.1 and Theorem 10.1 that the corresponding multiplication map in $\mathfrak{S t}$, namely $\mathfrak{U}_{\alpha_{1}} \times \cdots \times \mathfrak{U}_{\alpha_{n}} \rightarrow \mathfrak{U}_{\Psi}$, is bijective. Since $G_{\Psi} \rightarrow \mathfrak{U}_{\Psi}$ is surjective, it must also be bijective, hence an isomorphism. Now, if $\alpha$ and $\beta$ are a prenilpotent pair of roots then we may choose a chamber in neither of them and a chamber in both of them. We join these chambers by a minimal gallery $\left(C_{0}, \ldots, C_{n}\right)$. As mentioned above, the corresponding nilpotent set $\Psi$ of roots consists of all roots 
which contain $C_{n}$ but not $C_{0}$. In particular, $\Psi$ contains $\alpha$ and $\beta$. We have shown that $G_{\Psi} \rightarrow \mathfrak{U}_{\Psi}$ is an isomorphism. Since the Chevalley relation of $\alpha$ and $\beta$ holds in $\mathfrak{U}_{\Psi}$ (by the definition of $\mathfrak{U}_{\Psi}$ ), it holds in $G_{\Psi}$ too. This shows that the Chevalley relations of all prenilpotent pairs hold in $\mathfrak{P S t}$, so $\mathfrak{P S t} \rightarrow \mathfrak{S t}$ is an isomorphism, finishing the proof.

It remains to prove $\left(N_{n}\right)$. First we treat a special case that does not require induction. By hypothesis, $A$ is $S$-spherical, where $S$ is 2 (resp. 3) for part (iv) (resp. (iii)) of the theorem. To avoid degeneracies we suppose $\mathrm{rk} A>S$; the case rk $A \leq S$ is trivial because then $A$ is spherical and the isomorphism $\mathfrak{P S t} \rightarrow \mathfrak{S t}$ is tautological. Suppose that all the chambers in some minimal gallery $\left(C_{0}, \ldots, C_{n}\right)$ have a face $F$ with codimension $\leq S$ in common. By $S$-sphericity, the mirrors $\alpha^{\perp}$ of only finitely many $\alpha \in \Phi$ contain $F$. Therefore, any pair from $\alpha_{1}, \ldots, \alpha_{n}$ is classically prenilpotent. Their Chevalley relations hold in $\mathfrak{P S t}$ by definition. The fact that $\mathfrak{U}_{\alpha_{n}}$ normalizes $G_{\Psi_{0}}$ in $G_{\Psi}$ follows from these relations.

Now, for any minimal gallery of length $n \leq S$, its chambers have a face of codimension $n \leq S$ in common. (It is a subset of $\alpha_{1}^{\perp} \cap \cdots \cap \alpha_{n}^{\perp}$.) So the previous paragraph applies. This proves $\left(\boldsymbol{N}_{n}\right)$ for $n \leq S$, which we take as the base case of our induction. For the inductive step we take $n>S$, assume $\left(N_{1}\right), \ldots,\left(N_{n-1}\right)$, and suppose $\left(C_{0}, \ldots, C_{n}\right)$ is a minimal gallery. For $1 \leq k \leq l \leq n$ we write $G_{k, l}$ for

$$
\left\langle\mathfrak{U}_{\alpha_{k}}, \ldots, \mathfrak{U}_{\alpha_{l}}\right\rangle \subseteq \mathfrak{P S t} .
$$

We must show that $\mathfrak{U}_{\alpha_{n}}$ normalizes $G_{1, n-1}$.

Consider the subgallery $\left(C_{n-S}, \ldots, C_{n}\right)$ of length $S$. These chambers have a codimension- $S$ face $F$ in common. Write $W_{F}$ for its $W$-stabilizer, which is finite by $S$-sphericity. Among all chambers having $F$ as a face, let $D$ be the one closest to $C_{0}$. By [Abramenko and Brown 2008, Proposition 5.34] it is unique and there is a minimal gallery from $C_{0}$ to $C_{n-1}$ having $D$ as one of its terms, such that every chamber from $D$ to $C_{n-1}$ contains $F$. By replacing the subgallery $\left(C_{0}, \ldots, C_{n-1}\right)$ of our original minimal gallery with this one, we may suppose without loss of generality that $D=C_{m}$ for some $0 \leq m \leq n-S$ and that $C_{m}, \ldots, C_{n}$ all contain $F$. (This replacement may change the ordering on $\Psi_{0}=\left\{\alpha_{1}, \ldots, \alpha_{n-1}\right\}$, which is harmless.) The special case shows that $\mathfrak{U}_{\alpha_{n}}$ normalizes $G_{m+1, n-1}$. So it suffices to show that $\mathfrak{U}_{\alpha_{n}}$ also normalizes $G_{1, m}$.

At this point we specialize to proving part (iv) of the theorem. In this case $F$ has codimension 2. There are two chambers adjacent to $C_{m}$ that contain $F$. One is $C_{m+1}$ and we call the other one $C_{m+1}^{\prime}$. We write $\alpha_{m+1}^{\prime}$ for the root that contains $C_{m+1}^{\prime}$ but not $C_{m}$. Recall that $C_{m}$ was the unique chamber closest to $C_{0}$, of all those containing $F$. It follows that $\left(C_{0}, \ldots, C_{m}, C_{m+1}^{\prime}\right)$ is a minimal gallery. By a double application of $\left(N_{m+1}\right)$, which we may use because $m \leq n-S=n-2$, both $\mathfrak{U}_{\alpha_{m+1}}$ and $\mathfrak{U}_{\alpha_{m+1}^{\prime}}$ normalize $G_{1, m}$. Since $\alpha_{m+1}$ and $\alpha_{m+1}^{\prime}$ are simple roots for $W_{F}$, and $\alpha_{n}$ is 
positive with respect to them, Lemma 11.1 shows that $\mathfrak{U}_{\alpha_{n}}$ lies in $\left\langle\mathfrak{U}_{\alpha_{m+1}}, \mathfrak{U}_{\alpha_{m+1}^{\prime}}\right\rangle$. This uses the hypotheses on $R$ to deal with the possibility that $W_{F}$ has type $B_{2}$ or $G_{2}$. Therefore, $\mathfrak{U}_{\alpha_{n}}$ normalizes $G_{1, m}$, completing the proof of part (iv).

Now we prove part (iii). $F$ has codimension 3. So there are three chambers adjacent to $C_{m}$ that contain $F$. Write $C_{m+1}^{\prime}$ for any one of them (possibly $C_{m+1}$ ) and define $\beta$ as the root containing $C_{m+1}^{\prime}$ but not $C_{m}$. The three possibilities for $\beta$ form a system $\Sigma$ of simple roots for $W_{F}$. With respect to $\Sigma$, the positive roots of $W_{F}$ are exactly the ones that do not contain $C_{m}$, for example, $\alpha_{n}$.

There are two chambers adjacent to $C_{m+1}^{\prime}$ that contain $F$, besides $C_{m}$. Write $C_{m+2}^{\prime}$ for either of them and $\gamma$ for the root containing $C_{m+2}^{\prime}$ but not $C_{m+1}^{\prime}$. Because $C_{m}$ is the unique chamber containing $F$ that is closest to $C_{0},\left(C_{0}, \ldots, C_{m}, C_{m+1}^{\prime}, C_{m+2}^{\prime}\right)$ is a minimal gallery. In particular, $\gamma$ is a positive root with respect to $\Sigma$.

We claim that $\mathfrak{U}_{\beta}$ and $\mathfrak{U}_{\gamma}$ normalize $G_{1, m}$. For $\beta$ this is just induction using $\left(\boldsymbol{N}_{m+1}\right)$. For $\gamma$, we appeal to $\left(\boldsymbol{N}_{m+2}\right)$, but all this tells us is that $\mathfrak{U}_{\gamma}$ normalizes $\left\langle\mathfrak{U}_{\beta}, G_{1, m}\right\rangle$. In particular, it conjugates $G_{1, m}$ into this larger group. To show that $\mathfrak{U}_{\gamma}$ normalizes $G_{1, m}$ it suffices to show for every $k=1, \ldots, m$ that the Chevalley relation for $\gamma$ and $\alpha_{k}$ has no $\mathfrak{U}_{\beta}$ term. That is, it suffices to show that $\beta \notin \theta\left(\alpha_{k}, \gamma\right)$. Suppose to the contrary. Then $\beta$ is an $\mathbb{N}$-linear combination of $\alpha_{k}$ and $\gamma$. So $\alpha_{k}$ is a $\mathbb{Q}$-linear combination of $\beta$ and $\gamma$, and in particular its mirror contains $F$. Of the Weyl chambers for $W_{F}$, the one containing $C_{0}$ is the same as the one containing $C_{m}$, since $C_{m}$ is as close as possible to $C_{0}$. Since $\alpha_{k}$ does not contain $C_{0}$, it does not contain $C_{m}$ either. So, as a root of $W_{F}$, it is positive with respect to $\Sigma$. Now we have the contradiction that the simple root $\beta$ of $W_{F}$ is an $\mathbb{N}$-linear combination of the positive roots $\alpha_{k}$ and $\gamma$. This proves $\beta \notin \theta\left(\alpha_{k}, \gamma\right)$, so $\mathfrak{U}_{\gamma}$ normalizes $G_{1, m}$.

We have proven that $\mathfrak{U}_{\beta}$ and $\mathfrak{U}_{\gamma}$ normalize $G_{1, m}$. Letting $\beta$ and $\gamma$ vary over all possibilities gives all the roots called $\beta_{i}$ and $\gamma_{i, j}$ in Lemma 11.2. By that lemma, the group generated by these root groups contains the root groups of all positive roots of $W_{F}$. In particular, $\mathfrak{U}_{\alpha_{n}}$ normalizes $G_{1, m}$, as desired. This completes the proof of (iii).

\section{Finite presentations}

In this section we prove Theorems 1.4 and 1.5: pre-Steinberg groups, Steinberg groups and Kac-Moody groups are finitely presented under various hypotheses. Our strategy is to first prove parts (ii)-(iii) of Theorem 1.4, and then prove part (i) together with Theorem 1.5.

For use in the proof of Theorem 1.4(ii)-(iii), we recall the following result of Splitthoff, which grew from earlier work of Rehmann and Soulé [1976]. Then we prove Theorem 12.2, addressing finite generation rather than finite presentation, using his methods. Then we will prove Theorem 1.4(ii)-(iii). 
Theorem 12.1 [Splitthoff 1986, Theorem I]. Suppose $R$ is a commutative ring and $A$ is one of the ABCDEFG Dynkin diagrams. If either

(i) rk $A \geq 3$ and $R$ is finitely generated as a ring, or

(ii) $\operatorname{rk} A \geq 2$ and $R$ is finitely generated as a module over a subring generated by finitely many units,

then $\mathfrak{S t}_{A}(R)$ is finitely presented.

Theorem 12.2. Suppose $R$ is a commutative ring and $A$ is one of the ABCDEFG Dynkin diagrams. If either

(i) $\mathrm{rk} A \geq 2$ and $R$ is finitely generated as a ring, or

(ii) $\mathrm{rk} A \geq 1$ and $R$ is finitely generated as a module over a subring generated by finitely many units,

then $\mathfrak{S t}_{A}(R)$ is finitely generated.

Proof. In light of Splitthoff's theorem, it suffices to treat the cases $A=A_{2}, B_{2}, G_{2}$ in (i) and the case $A=A_{1}$ in (ii). For (i) it suffices to treat the case $R=\mathbb{Z}\left[z_{1}, \ldots, z_{n}\right]$, since $\mathfrak{S t}_{A}(R) \rightarrow \mathfrak{S t}_{A}(R / I)$ is surjective for any ideal $I$. In the rest of the proof we abbreviate $\mathfrak{S t}_{A}(R)$ to $\mathfrak{S t}$. Keeping our standard notation, $\Phi$ is the root system, and $\mathfrak{S t}$ is generated by groups $\mathfrak{U}_{\alpha} \cong R$ with $\alpha$ varying over $\Phi$. As discussed in Section 6, writing down elements $X_{\alpha}(t)$ of $\mathfrak{U}_{\alpha}$ requires choosing one of the two elements of $E_{\alpha}$, but the sign issues coming from this choice will not affect the proof. For each $p \geq 1$ we write $\mathfrak{U}_{\alpha, p}$ for the subgroup of $\mathfrak{U}_{\alpha}$ consisting of all $X_{\alpha}(t)$ where $t \in R$ is a polynomial of degree $\leq p$.

$A_{2}$ case: If $\alpha, \beta \in \Phi$ make angle $2 \pi / 3$ then their Chevalley relation reads

$$
\left[X_{\alpha}(t), X_{\beta}(u)\right]=X_{\alpha+\beta}( \pm t u),
$$

where the unimportant sign depends on the choices of elements of $E_{\alpha}, E_{\beta}$ and $E_{\alpha+\beta}$. It follows that $\left[\mathfrak{U}_{\alpha, p}, \mathfrak{U}_{\beta, q}\right]$ contains $\mathfrak{U}_{\alpha+\beta, p+q}$. An easy induction shows that $\mathfrak{S t}$ is generated by the $\mathfrak{U}_{\alpha, 1} \cong \mathbb{Z}^{n+1}$, with $\alpha$ varying over $\Phi$.

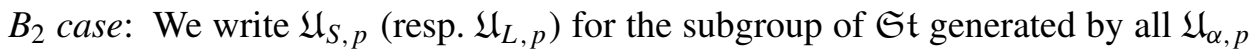
with $\alpha$ a short (resp. long root). If $\sigma, \lambda$ are short and long roots with angle $3 \pi / 4$, then we recall their Chevalley relation from (7-16) as

$$
\left[X_{\sigma}(t), X_{\lambda}(u)\right]=X_{\lambda+\sigma}(-t u) X_{\lambda+2 \sigma}\left(t^{2} u\right) .
$$

Here we have implicitly chosen some elements of $E_{\sigma}, E_{\lambda}, E_{\lambda+\sigma}$ and $E_{\lambda+2 \sigma}$ so that one can write down the relation explicitly. Note that the first term on the right lies in a short root group and the second lies in a long root group. Recall that $n$ is the number of variables in the polynomial ring $R$. We claim that $\mathfrak{S t}$ equals $\left\langle\mathfrak{U}_{S, n}, \mathfrak{U}_{L, n+2}\right\rangle$ and is therefore finitely generated. The case $n=0$ is trivial, 
so suppose $n>0$. Our claim follows from induction using the following two ingredients.

First, for any $p \geq 1,\left\langle\mathfrak{U}_{S, p}, \mathfrak{U}_{L, p+2}\right\rangle$ contains $\mathfrak{U}_{S, p+1}$. To see this let $g \in R$ be any monomial of degree $p+1$ and write it as $t u$ for monomials $t, u \in R$ of degrees 1 and $p$. Then (12-1) yields

$$
X_{\lambda+\sigma}(g)=X_{\lambda+2 \sigma}\left(t^{2} u\right)\left[X_{\lambda}(u), X_{\sigma}(t)\right] \in \mathfrak{U}_{L, p+2} \cdot\left[\mathfrak{U}_{L, p}, \mathfrak{U}_{S, 1}\right] .
$$

Letting $g$ vary shows that $\mathfrak{U}_{\lambda+\sigma, p+1} \subseteq\left\langle\mathfrak{U}_{S, p}, \mathfrak{U}_{L, p+2}\right\rangle$. Then letting $\sigma, \lambda$ vary over all pairs of roots making angle $3 \pi / 4$, so that $\lambda+\sigma$ varies over all short roots, shows that $\mathfrak{U}_{S, p+1} \subseteq\left\langle\mathfrak{U}_{S, p}, \mathfrak{U}_{L, p+2}\right\rangle$, as desired.

Second, for any $p \geq n,\left\langle\mathfrak{U}_{S, p+1}, \mathfrak{U}_{L, p+2}\right\rangle$ contains $\mathfrak{U}_{L, p+3}$. To see this let $g \in R$ be any monomial of degree $p+3$ and write it as $t^{2} u$ for monomials $t, u \in R$ of degrees 2 and $p-1$. This is possible because $p+3$ is at least 3 more than the number of variables in the polynomial ring $R$. Then (12-1) can be written

$$
X_{\lambda+2 \sigma}(g)=X_{\lambda+\sigma}(t u)\left[X_{\sigma}(t), X_{\lambda}(u)\right] \in \mathfrak{U}_{S, p+1} \cdot\left[\mathfrak{U}_{S, 2}, \mathfrak{U}_{L, p-1}\right] .
$$

Varying $g$ and the pair $(\sigma, \lambda)$ as in the previous paragraph establishes

$$
\mathfrak{U}_{L, p+3} \subseteq\left\langle\mathfrak{U}_{S, p+1}, \mathfrak{U}_{L, p+2}\right\rangle .
$$

$G_{2}$ case: Defining $\mathfrak{U}_{S, p}$ and $\mathfrak{U}_{L, p}$ as in the $B_{2}$ case, it suffices to show that $\mathfrak{S t}$ equals $\left\langle\mathfrak{U}_{L, 1}, \mathfrak{U}_{S, n}\right\rangle$. The $A_{2}$ case shows that $\mathfrak{U}_{L, 1}$ equals the union $\mathfrak{U}_{L, \infty}$ of all the $\mathfrak{U}_{L, p}$. So it suffices to prove that if $p \geq n$ then $\left\langle\mathfrak{U}_{L, \infty}, \mathfrak{U}_{S, p}\right\rangle$ contains $\mathfrak{U}_{S, p+1}$. If $\sigma, \lambda \in \Phi$ are short and long simple roots then their Chevalley relation (7-23) can be written

$$
\left[X_{\sigma}(t), X_{\lambda}(u)\right]=X_{\sigma^{\prime \prime}}\left(t^{2} u\right) X_{\sigma^{\prime}}(-t u) \cdot(\text { long-root-group elements), }
$$

where $\sigma^{\prime}, \sigma^{\prime \prime}$ are the short roots $\sigma+\lambda$ and $2 \sigma+\lambda$. As before, we have implicitly chosen elements of $E_{\sigma}, E_{\lambda}, E_{\sigma^{\prime}}$ and $E_{\sigma^{\prime \prime}}$. Given any monomial $g \in R$ of degree $p+1$, by using $p+1>n$ we may write it as $t^{2} u$, where $t$ has degree 1 and $u$ has degree $p-1$. So every term in the Chevalley relation except $X_{\sigma^{\prime \prime}}\left(t^{2} u\right)$ lies in $\mathfrak{U}_{S, p}$ or $\mathfrak{U}_{L, \infty}$. Therefore, $\left\langle\mathfrak{U}_{S, p}, \mathfrak{U}_{L, \infty}\right\rangle$ contains $X_{\sigma^{\prime \prime}}(g)$, hence $\mathfrak{U}_{\sigma^{\prime \prime}, p+1}$ (by varying $g$ ), hence $\mathfrak{U}_{S, p+1}$ (by varying $\sigma$ and $\lambda$ so that $\sigma^{\prime \prime}$ varies over the short roots).

$A_{1}$ case: in this case we are assuming there exist units $x_{1}, \ldots, x_{n}$ of $R$ and a finite set $Y$ of generators for $R$ as a module over $\mathbb{Z}\left[x_{1}^{ \pm 1}, \ldots, x_{n}^{ \pm}\right]$. We suppose without loss that $Y$ contains 1. We use the description of $\mathfrak{S t}_{A_{1}}$ from Section 2, and write $G$ for the subgroup generated by $S$ and the $X\left(x_{1}^{m_{1}} \cdots x_{n}^{m_{n}} y\right)$ with $m_{1}, \ldots, m_{n} \in$ $\{0, \pm 1\}$ and $y \in Y$. By construction, $G$ contains the $\tilde{s}\left(x_{k}^{ \pm 1}\right)$, and it contains $\tilde{s}(-1)$ since $Y$ contains 1 . Therefore, $G$ contains every $\tilde{h}\left(x_{k}^{ \pm 1}\right)$. Relation (2-4) shows that if $G$ contains $X(u)$ for some $u$, then it also contains every $X\left(x_{k}^{ \pm 2} u\right)$. It follows that $G$ contains every $X\left(x_{1}^{m_{1}} \cdots x_{n}^{m_{n}} y\right)$ with $m_{1}, \ldots, m_{n} \in \mathbb{Z}$. Therefore, $G=\mathfrak{S t}$. 
Proof of Theorem 1.4(ii)-(iii). We abbreviate $\mathfrak{P S t}_{A}(R)$ to $\mathfrak{P S t}_{A}$. We begin with (ii), so $A$ is assumed 2-spherical without $A_{1}$ components, and $R$ is finitely generated as a module over a subring generated by finitely many units. We must show that $\mathfrak{P S t}_{A}$ is finitely presented. Let $G$ be the direct limit of the groups $\mathfrak{P S t}_{B}$ with $B$ varying over the singletons and irreducible rank-2 subdiagrams. By 2-sphericity, each $\mathfrak{P S t}_{B}$ is isomorphic to the corresponding $\mathfrak{S t}_{B}$. Also $G$ is generated by the images of the $\mathfrak{S t}_{B}$ with $|B|=2$, because every singleton lies in some irreducible rank-2 diagram. By Splitthoff's theorem, each of these $\mathfrak{S t}_{B}$ is finitely presented. And Theorem 12.2 shows that each $\mathfrak{S t}_{B}$ with $|B|=1$ is finitely generated. Therefore, the direct limit $G$ is finitely presented.

Now we consider all $A_{1} A_{1}$ subdiagrams $\{i, j\}$ of $A$. For each of them we impose on $G$ the relations that (the images in $G$ of) $\mathfrak{S t}_{\{i\}}$ and $\mathfrak{S t}_{\{j\}}$ commute. Because these two groups are finitely generated (Theorem 12.2 again), this can be done with finitely many relations. This finitely presented quotient of $G$ is then the direct limit of the groups $\mathfrak{S t}_{B}$ with $B$ varying over all subdiagrams of $A$ of rank $\leq 2$. Again using 2-sphericity, we can replace the $\mathfrak{S t}_{B}$ 's by $\mathfrak{P S t}_{B}$ 's. Then Corollary 1.3 says that the direct limit is $\mathfrak{P S t}_{A}$. This finishes the proof of (ii).

Now we prove (iii), in which we are assuming $R$ is a finitely generated ring. Consider the direct limit of the groups $\mathfrak{P S t}_{B}$ with $B$ varying over the irreducible spherical subdiagrams of rank $\geq 2$. Because every node and every pair of nodes lies in such a subdiagram, this direct limit is the same as $\mathfrak{P S t}_{A}$. Because every $B$ is spherical, we may replace the groups $\mathfrak{P S t}_{B}$ by $\mathfrak{S t}_{B}$. By hypothesis on $A$, $G$ is generated by the $\mathfrak{S t}_{B}$ with $|B|>2$, which are finitely presented by Splitthoff's theorem. And Theorem 12.2 shows that those with $|B|=2$ are finitely generated. So the direct limit is finitely presented.

Now we turn to Kac-Moody groups. For our purposes, Tits' Kac-Moody group $\mathfrak{G}_{A}(R)$ may be defined as the quotient of $\mathfrak{S t}_{A}(R)$ by the subgroup normally generated by the relators

$$
\tilde{h}_{i}(u) \tilde{h}_{i}(v) \cdot \tilde{h}_{i}(u v)^{-1}
$$

with $i \in I$ and $u, v \in R^{*}$. See [Rémy 2002, §8.3.3] or [Tits 1987, §3.6] for the more general construction of $\mathfrak{G}_{D}(R)$ from a root datum $D$. In the rest of this section, $R^{*}$ will be finitely generated, and under this hypothesis the choice of root datum has no effect on whether $\mathfrak{G}_{D}(R)$ is finitely presented. (We are using the root datum which Rémy calls simply connected [2002, §7.1.2] and Tits calls "simply connected in the strong sense" [1987, Remark 3.7(c)].)

The following technical lemma shows that when $R^{*}$ is finitely generated, killing a finite set of relators (12-2) kills all the rest too. The reason it assumes only some of the relations present in $\mathfrak{P S t}_{A}(R)$ is so we can use it in the proof of Theorem 1.4(i). 
There, the goal is to deduce the full presentation of $\mathfrak{P S t}_{A}(R)$ from just some of its relations.

Lemma 12.3. Suppose $R$ is a commutative ring and $r_{1}, \ldots, r_{m}$ are generators for $R^{*}$, closed under inversion. Suppose $G$ is the group with generators $S$ and $X(t)$, with $t \in R$, subject to the relations

$$
\begin{gathered}
\tilde{h}(r) X(t) \tilde{h}(r)^{-1}=X\left(r^{2} t\right), \\
\tilde{h}(r) S X(t) S^{-1} \tilde{h}(r)^{-1}=S X\left(t / r^{2}\right) S^{-1},
\end{gathered}
$$

for all $r=r_{1}, \ldots, r_{m}$ and all $t \in R$, where

$$
\tilde{h}(r):=\tilde{s}(r) \tilde{s}(1)^{-1} \quad \text { and } \quad \tilde{s}(r):=X(r) S X(1 / r) S^{-1} X(r) .
$$

Then all $\mathcal{P}_{u, v}:=\tilde{h}(u v) \tilde{h}(u)^{-1} \tilde{h}(v)^{-1}$, with $u, v \in R^{*}$, lie in the subgroup of $G$ normally generated by some finite set of them.

Proof. Define $N$ as the subgroup of $G$ normally generated by the following finite set of $\mathcal{P}_{u, v}$ :

$$
\tilde{h}\left(r_{k} r_{1}^{p_{1}} \cdots r_{m}^{p_{m}}\right) \cdot \tilde{h}\left(r_{1}^{p_{1}} \cdots r_{m}^{p_{m}}\right)^{-1} \tilde{h}\left(r_{k}\right)^{-1},
$$

with $k=1, \ldots, m$ and $p_{1}, \ldots, p_{m} \in\{0,1\}$. We write $\equiv$ to indicate equality modulo $N$. As special cases we have $\left[\tilde{h}\left(r_{k}\right), \tilde{h}\left(r_{l}\right)\right] \equiv 1, \tilde{h}\left(r_{k}^{2}\right) \equiv \tilde{h}\left(r_{k}\right)^{2}$, and that if $p_{1}, \ldots, p_{m} \in\{0,1\}$ then $\tilde{h}\left(r_{1}^{p_{1}} \cdots r_{m}^{p_{m}}\right)$ lies in the abelian subgroup $Y$ of $G / N$ generated by $\tilde{h}\left(r_{1}\right), \ldots, \tilde{h}\left(r_{m}\right)$.

We claim that every $\mathcal{P}_{u, v}$ lies in $Y$. Since $Y$ is finitely generated abelian, we may therefore kill all the $\mathcal{P}_{u, v}$ 's by killing some finite set of them, proving the theorem. To prove the claim it suffices to show that every $\tilde{h}(u)$ lies in $Y$, which we do by induction. That is, supposing $\tilde{h}(u) \in Y$ we will prove $\tilde{h}\left(r_{k}^{2} u\right) \in Y$ for each $k=1, \ldots, m$. The following calculations in $G$ mimic the proof of (6-12), paying close attention to which relations are used. First, (12-3)-(12-4) imply

$$
\tilde{h}\left(r_{k}\right) \tilde{s}(u) \tilde{h}\left(r_{k}\right)^{-1}=\tilde{s}\left(r_{k}^{2} u\right) .
$$

From the definition of $\tilde{h}(u)$ we get

$$
\tilde{h}\left(r_{k}\right) \tilde{h}(u) \tilde{h}\left(r_{k}\right)^{-1}=\tilde{h}\left(r_{k}^{2} u\right) \tilde{h}\left(r_{k}^{2}\right)^{-1} .
$$

Right-multiplying by $\tilde{h}(u)^{-1}$ yields $\left[\tilde{h}\left(r_{k}\right), \tilde{h}(u)\right]=\mathcal{P}_{r_{k}^{2}, u}$. Now, $\tilde{h}(u) \in Y$ implies $\left[\tilde{h}\left(r_{k}\right), \tilde{h}(u)\right] \equiv 1$, so $\mathcal{P}_{r_{k}^{2}, u} \equiv 1$, so $\tilde{h}\left(r_{k}^{2} u\right) \equiv \tilde{h}(u) \tilde{h}\left(r_{k}^{2}\right) \in Y$, as desired.

Corollary 12.4. Suppose $R$ is a commutative ring with finitely generated unit group $R^{*}$, and $A$ is any generalized Cartan matrix. Then the subgroup of $\mathfrak{P S t}_{A}(R)$ normally generated by all relators (12-2) is normally generated by finitely many of them. 
Proof of Theorem 1.5. We must show that $\mathfrak{G}_{D}(R)$ is finitely presented, assuming that $\mathfrak{S t}_{A}(R)$ is and that $R^{*}$ is finitely generated. For $\mathfrak{G}_{A}(R)$ this is immediate from Corollary 12.4. Also, its subgroup $H$ generated by the images of the $\tilde{h}_{i}(r)$ with $i \in I$ and $r \in R^{*}$ is finitely generated abelian. For a general root datum $D$, one obtains $\mathfrak{G}_{D}(R)$ by the following construction. First one quotients $\mathfrak{G}_{A}(R)$ by a subgroup of $H$. Then one takes the semidirect product of this by a torus $\mathcal{T}$ (a copy of $\left.\left(R^{*}\right)^{n}\right)$. Then one identifies the generators of $H$ with certain elements of $\mathcal{T}$. Since $R^{*}$ is finitely generated, none of these steps affects finite presentability.

Proof of Theorem 1.4(i). We must show that if $R$ is finitely generated as an abelian group, then $\mathfrak{P S t}_{A}(R)$ is finitely presented for any generalized Cartan matrix $A$. Suppose $R$ is generated as an abelian group by $t_{1}, \ldots, t_{n}$. Then $\mathfrak{P S t}_{A}(R)$ is generated by the $S_{i}$ and $X_{i}\left(t_{k}\right)$, so it is finitely generated. Because $R$ is finitely generated as an abelian group, its multiplicative group $R^{*}$ is also. At its heart, this is the Dirichlet unit theorem. See [Lang 1983, Corollary 7.5] for the full result. Let $r_{1}, \ldots, r_{m}$ be a set of generators for $R^{*}$, closed under inversion.

Let $N$ be the central subgroup of $\mathfrak{P S t}_{A}(R)$ normally generated by all relators (12-2). It is elementary and well-known that if a group is finitely generated, and a central quotient of it is finitely presented, then it is itself finitely presented. (See [Johnson 1997, §10.2] for the required background.) Therefore, the finite presentability of $\mathfrak{P S t}_{A}(R)$ will follow from that of $\mathfrak{P S t}_{A}(R) / N$. The relators defining the latter group are (7-1)-(7-26) and (12-2). We will show that finitely many of them imply all the others.

In the definition of $\widehat{W}$, there are only finitely many relations (7-1)-(7-3). The addition rules (7-4) in $\mathfrak{U}_{i} \cong R$ can be got by imposing finitely many relations on the $X_{i}\left(t_{k}\right)$. Relations (7-5)-(7-9) describe how certain words in the $S_{i}$ conjugate arbitrary $X_{j}(t)$. By the additivity of $X_{j}(t)$ in $t$, it suffices to impose only those with $t$ among $t_{1}, \ldots, t_{n}$. The Chevalley relations (7-10)-(7-23) may be imposed using only finitely many relations, because the Borel subgroup of any rank-2 Chevalley group over $R$ is polycyclic (since $R$ is).

Now for the tricky step: we impose relations (7-24)-(7-25) for $r=r_{1}, \ldots, r_{m}$ and $t=t_{1}, \ldots, t_{n}$. The additivity of $X_{j}(t)$ in $t$ implies these relations for $r=r_{1}, \ldots, r_{m}$ and arbitrary $t \in R$. These are exactly the relations (12-3)-(12-4) assumed in the statement of Lemma 12.3. That lemma shows that we may impose all the relations (12-2) by imposing some finite number of them. Working modulo these, $\tilde{h}_{i}(r)$ is multiplicative in $r$, for each $i$. Therefore, our relations (7-24)-(7-25) for $r=r_{1}, \ldots, r_{m}$ imply the same relations for arbitrary $r$.

Starting with the generators $S_{i}, X_{i}(t)$, with $i \in I$ and $t=t_{1}, \ldots, t_{n}$, we have found finitely many relations from (7-1)-(7-26) and (12-2) that imply all the others. Therefore, $\mathfrak{P S t}_{A}(R) / N$ is finitely presented, so the same holds for $\mathfrak{P S t}_{A}(R)$ itself. 


\section{Acknowledgements}

The author is very grateful to the Japan Society for the Promotion of Science and to Kyoto University, for their support and hospitality, and to Lisa Carbone, for getting him interested in Kac-Moody groups over $\mathbb{Z}$.

\section{References}

[Abramenko and Brown 2008] P. Abramenko and K. S. Brown, Buildings: theory and applications, Graduate Texts in Mathematics 248, Springer, New York, 2008. MR 2439729 Zbl 1214.20033

[Abramenko and Mühlherr 1997] P. Abramenko and B. Mühlherr, "Présentations de certaines BNpaires jumelées comme sommes amalgamées”, C. R. Acad. Sci. Paris Sér. I Math. 325:7 (1997), 701-706. MR 1483702 Zbl 0934.20024

[Allcock 2013] D. Allcock, "Reflection centralizers in Coxeter groups", Transform. Groups 18:3 (2013), 599-613. MR 3084328 Zbl 1283.20042

[Allcock 2016] D. Allcock, "Presentation of affine Kac-Moody groups over rings", Algebra Number Theory 10:3 (2016), 533-556. MR 3513130 Zbl 06596319

[Allcock and Carbone 2016] D. Allcock and L. Carbone, "Presentation of hyperbolic Kac-Moody groups over rings", J. Algebra 445 (2016), 232-243. MR 3418056 Zbl 1333.20050

[Borcherds 1998] R. E. Borcherds, "Coxeter groups, Lorentzian lattices, and K3 surfaces", Internat. Math. Res. Notices 19 (1998), 1011-1031. MR 1654763 Zbl 0935.20027

[Bourbaki 1975] N. Bourbaki, Éléments de mathématique, XXXVIII: Groupes et algèbres de Lie (Chapitre VII: Sous-algèbres de Cartan, éléments réguliers; Chapitre VIII: Algèbres de Lie semisimples déployées), Actualités Scientifiques et Industrielles, No. 1364., Hermann, Paris, 1975. MR 0453824 Zbl 0329.17002

[Bourbaki 2002] N. Bourbaki, Lie groups and Lie algebras, Chapters 4-6, Springer, Berlin, 2002. MR 1890629 Zbl 0983.17001

[Brink 1996] B. Brink, "On centralizers of reflections in Coxeter groups", Bull. London Math. Soc. 28:5 (1996), 465-470. MR 1396145 Zbl 0852.20033

[Brink and Howlett 1999] B. Brink and R. B. Howlett, "Normalizers of parabolic subgroups in Coxeter groups", Invent. Math. 136:2 (1999), 323-351. MR 1688445 Zbl 0926.20024

[Caprace and Rémy 2009] P.-E. Caprace and B. Rémy, "Groups with a root group datum”, Innov. Incidence Geom. 9 (2009), 5-77. MR 2658894 Zbl 1221.20021

[Carter 1972] R. W. Carter, Simple groups of Lie type, Pure and Applied Mathematics 28, John Wiley \& Sons, London-New York-Sydney, 1972. MR 0407163 Zbl 0248.20015

[Dennis and Stein 1974] R. K. Dennis and M. R. Stein, "Injective stability for $K_{2}$ of local rings", Bull. Amer. Math. Soc. 80 (1974), 1010-1013. MR 0371880 Zbl 0299.18007

[Garland 1978] H. Garland, “The arithmetic theory of loop algebras”, J. Algebra 53:2 (1978), 480551. Correction in 63:1 (1980), 285. MR 502647 Zbl 0383.17012

[Hée 1990] J.-Y. Hée, "Construction de groupes tordus en théorie de Kac-Moody", C. R. Acad. Sci. Paris Sér. I Math. 310:3 (1990), 77-80. MR 1044619 Zbl 0707.22008

[Hée 2008] J.-Y. Hée, “Torsion de groupes munis d'une donnée radicielle”, J. Algebra 319:11 (2008), 4738-4758. MR 2416741 Zbl 1148.22016

[Johnson 1997] D. L. Johnson, Presentations of groups, 2nd ed., London Mathematical Society Student Texts 15, Cambridge University Press, 1997. MR 1472735 Zbl 0906.20019

[Kac 1990] V. G. Kac, Infinite-dimensional Lie algebras, 3rd ed., Cambridge University Press, 1990. MR 1104219 Zbl 0716.17022 
[Kiralis et al. 1996] G. Kiralis, S. Krstić, and J. McCool, "Finite presentability of $\Phi_{n}(G), \mathrm{GL}_{n}(\mathbf{Z} G)$ and their elementary subgroups and Steinberg groups", Proc. London Math. Soc. (3) 73:3 (1996), 575-622. MR 1407462 Zbl 0865.20025

[Kostant 1966] B. Kostant, "Groups over Z”, pp. 90-98 in Algebraic Groups and Discontinuous Subgroups (Boulder, CO, 1965), edited by A. Borel and G. D. Mostow, Amer. Math. Soc., Providence, RI, 1966. MR 0207713 Zbl 0199.06903

[Lang 1983] S. Lang, Fundamentals of Diophantine geometry, Springer, New York, 1983. MR 715605 Zbl 0528.14013

[Li 1989] F. A. Li, "Finite presentability of Steinberg groups over group rings", Acta Math. Sinica (N.S.) 5:4 (1989), 297-301. MR 1037850 Zbl 0688.16028

[Morita and Rehmann 1990] J. Morita and U. Rehmann, "A Matsumoto-type theorem for Kac-Moody groups", Tohoku Math. J. (2) 42:4 (1990), 537-560. MR 1076175 Zbl 0701.19001

[Rehmann and Soulé 1976] U. Rehmann and C. Soulé, "Finitely presented groups of matrices", pp. 164-169. Lecture Notes in Math., Vol. 551 in Algebraic K-theory (Northwestern Univ., Evanston, IL, 1976), edited by M. R. Stein, Springer, Berlin, 1976. MR 0486175 Zbl 0445.20025

[Rémy 2002] B. Rémy, Groupes de Kac-Moody déployés et presque déployés, Astérisque 277, Société Mathématique de France, Paris, 2002. MR 1909671 Zbl 1001.22018

[SGA 3 III 1970] M. Demazure and A. Grothendieck, Schémas en groupes, Tome III: Structure des schémas en groupes réductifs, Exposés XIX-XXVI (Séminaire de Géométrie Algébrique du Bois Marie 1962-1964), Lecture Notes in Math. 153, Springer, Berlin, 1970. MR 43 \#223c Zbl 0212.52810

[Splitthoff 1986] S. Splitthoff, "Finite presentability of Steinberg groups and related Chevalley groups", pp. 635-687 in Applications of algebraic K-theory to algebraic geometry and number theory, Part II (Boulder, CO, 1983), edited by S. J. Bloch et al., Contemp. Math. 55, Amer. Math. Soc., Providence, RI, 1986. MR 862658 Zbl 0596.20034

[Steinberg 1968] R. Steinberg, Lectures on Chevalley groups, edited by J. Faulkner and R. Wilson, Yale University, New Haven, CT, 1968. MR 0466335 Zbl 1196.22001

[Tits 1966a] J. Tits, "Normalisateurs de tores, I: Groupes de Coxeter étendus”, J. Algebra 4 (1966), 96-116. MR 0206117 Zbl 0145.24703

[Tits 1966b] J. Tits, "Sur les constantes de structure et le théorème d'existence des algèbres de Lie semi-simples”, Inst. Hautes Études Sci. Publ. Math. 31 (1966), 21-58. MR 0214638 Zbl 0145.25804

[Tits 1974] J. Tits, Buildings of spherical type and finite BN-pairs, Lecture Notes in Mathematics 386, Springer, Berlin-New York, 1974. MR 0470099 Zbl 0295.20047

[Tits 1987] J. Tits, "Uniqueness and presentation of Kac-Moody groups over fields", J. Algebra 105:2 (1987), 542-573. MR 873684 Zbl 0626.22013

[Tits 1992] J. Tits, "Twin buildings and groups of Kac-Moody type", pp. 249-286 in Groups, combinatorics \& geometry (Durham, 1990), edited by M. Liebeck and J. Saxl, London Math. Soc. Lecture Note Ser. 165, Cambridge University Press, 1992. MR 1200265 Zbl 0851.22023

[Zhang 1991] H. Zhang, "Finite presentability of Steinberg group $\operatorname{St}_{n}(L \Pi)$ ", Northeast. Math. J. 7:3 (1991), 317-325. MR 1164086 Zbl 0767.19001

Communicated by Edward Frenkel

Received 2016-03-29 Accepted 2016-06-11

allcock@math.utexas.edu Department of Mathematics, University of Texas at Austin, RLM 8.100, 2515 Speedway Stop C1200, Austin, TX 78712, United States 


\section{Algebra \& Number Theory}

msp.org/ant

\section{EDITORS}

MANAGING EDITOR

Bjorn Poonen

Massachusetts Institute of Technology

Cambridge, USA

\author{
EDITORIAL BOARD CHAIR \\ David Eisenbud \\ University of California \\ Berkeley, USA
}

BOARD OF EDITORS

$\begin{aligned} \text { Dave Benson } & \text { University of Aberdeen, Scotland } & \text { Susan Montgomery } & \text { University of Southern California, USA } \\ \text { Richard E. Borcherds } & \text { University of California, Berkeley, USA } & \text { Shigefumi Mori } & \text { RIMS, Kyoto University, Japan } \\ \text { John H. Coates } & \text { University of Cambridge, UK } & \text { Raman Parimala } & \text { Emory University, USA } \\ \text { J-L. Colliot-Thélène } & \text { CNRS, Université Paris-Sud, France } & \text { Jonathan Pila } & \text { University of Oxford, UK } \\ \text { Brian D. Conrad } & \text { Stanford University, USA } & \text { Anand Pillay } & \text { University of Notre Dame, USA } \\ \text { Hélène Esnault } & \text { Freie Universität Berlin, Germany } & \text { Victor Reiner } & \text { University of Minnesota, USA } \\ \text { Hubert Flenner } & \text { Ruhr-Universität, Germany } & \text { Peter Sarnak } & \text { Princeton University, USA } \\ \text { Sergey Fomin } & \text { University of Michigan, USA } & \text { Joseph H. Silverman } & \text { Brown University, USA } \\ \text { Edward Frenkel } & \text { University of California, Berkeley, USA } & \text { Michael Singer } & \text { North Carolina State University, USA } \\ \text { Andrew Granville } & \text { Université de Montréal, Canada } & \text { Vasudevan Srinivas } & \text { Tata Inst. of Fund. Research, India } \\ \text { Joseph Gubeladze } & \text { San Francisco State University, USA } & \text { J. Toby Stafford } & \text { University of Michigan, USA } \\ \text { Roger Heath-Brown } & \text { Oxford University, UK } & \text { Ravi Vakil } & \text { Stanford University, USA } \\ \text { Craig Huneke } & \text { University of Virginia, USA } & \text { Michel van den Bergh } & \text { Hasselt University, Belgium } \\ \text { Kiran S. Kedlaya } & \text { Univ. of California, San Diego, USA } & \text { Marie-France Vignéras } & \text { Université Paris VII, France } \\ \text { János Kollár } & \text { Princeton University, USA } & \text { Kei-Ichi Watanabe } & \text { Nihon University, Japan } \\ \text { Yuri Manin } & \text { Northwestern University, USA } & \text { Efim Zelmanov } & \text { University of California, San Diego, USA } \\ \text { Philippe Michel } & \text { École Polytechnique Fédérale de Lausanne } & \text { Shou-Wu Zhang } & \text { Princeton University, USA }\end{aligned}$

PRODUCTION

production@msp.org

Silvio Levy, Scientific Editor

See inside back cover or msp.org/ant for submission instructions.

The subscription price for 2016 is US $\$ 290$ /year for the electronic version, and $\$ 485 /$ year (+\$55, if shipping outside the US) for print and electronic. Subscriptions, requests for back issues and changes of subscribers address should be sent to MSP.

Algebra \& Number Theory (ISSN 1944-7833 electronic, 1937-0652 printed) at Mathematical Sciences Publishers, 798 Evans Hall \#3840, c/o University of California, Berkeley, CA 94720-3840 is published continuously online. Periodical rate postage paid at Berkeley, CA 94704, and additional mailing offices.

ANT peer review and production are managed by EditFLow ${ }^{\circledR}$ from MSP.

\section{PUBLISHED BY}

- mathematical sciences publishers

nonprofit scientific publishing

http://msp.org/

() 2016 Mathematical Sciences Publishers 


\section{Algebra \& Number Theory}

Volume $10 \quad$ No. $8 \quad 2016$

Tropical independence, II: The maximal rank conjecture for quadrics

DAVID JENSEN and SAM PAYNE

Algebraicity of normal analytic compactifications of $\mathbb{C}^{2}$ with one irreducible curve at infinity

PINAKI MONDAL

The local lifting problem for $A_{4}$

ANDREW OBUS

Syntomic cohomology and $p$-adic regulators for varieties over $p$-adic fields

JAN NEKOVÁŘ and WIESŁAWA NIZIOŁ

Appendix: LAURENT BERGER and FRÉDÉRIC DÉGLISE

Steinberg groups as amalgams

DANIEL ALLCOCK 